LA W REN CE LIVERMORE NATIONAL LABORATORY

\section{Upwind schemes for the wave equation in second order form}

J. W. Banks, W. D. Henshaw

December 19, 2011

Journal of Computational Physics 
This document was prepared as an account of work sponsored by an agency of the United States government. Neither the United States government nor Lawrence Livermore National Security, LLC, nor any of their employees makes any warranty, expressed or implied, or assumes any legal liability or responsibility for the accuracy, completeness, or usefulness of any information, apparatus, product, or process disclosed, or represents that its use would not infringe privately owned rights. Reference herein to any specific commercial product, process, or service by trade name, trademark, manufacturer, or otherwise does not necessarily constitute or imply its endorsement, recommendation, or favoring by the United States government or Lawrence Livermore National Security, LLC. The views and opinions of authors expressed herein do not necessarily state or reflect those of the United States government or Lawrence Livermore National Security, LLC, and shall not be used for advertising or product endorsement purposes. 


\title{
Upwind schemes for the wave equation in second order form
}

\author{
Jeffrey W. Banks ${ }^{\mathrm{a}, 1, *}$, William D. Henshaw ${ }^{\mathrm{a}, 1}$ \\ ${ }^{a}$ Centre for Applied Scientific Computing, Lawrence Livermore National Laboratory, Livermore, CA 94551, USA
}

\begin{abstract}
We develop new high-order accurate upwind schemes for the wave equation in second-order form. These schemes are developed directly for the equations in second-order form, as opposed to transforming the equations to a first-order hyperbolic system. The schemes are based on the solution to a local Riemann-type problem that uses d'Alembert's exact solution. We construct conservative finite difference approximations, although finite volume approximations are also possible. High-order accuracy is obtained using a space-time procedure which requires only two discrete time levels. The advantages of our approach include efficiency in both memory and speed together with accuracy and robustness. The stability and accuracy of the approximations in one and two space dimensions are studied through normal-mode analysis. The form of the dissipation and dispersion introduced by the schemes is elucidated from the modified equations. Upwind schemes are implemented and verified in one dimension for approximations up to sixth-order accuracy, and in two dimensions for approximations up to fourth-order accuracy. Numerical computations demonstrate the attractive properties of the approach for solutions with varying degrees of smoothness.
\end{abstract}

Keywords: second-order wave equations, upwind discretization, Godunov methods, high-order accurate, finite-difference, finite-volume

\section{Contents}

1 Introduction

2 Construction of an upwind scheme for the second-order wave equation in one dimension 3

3 A general construction for high-order upwind schemes - the one-dimensional case 8

3.1 The upwind flux for the second-order system $\ldots \ldots \ldots \ldots \ldots$

3.2 A first-order accurate upwind scheme . . . . . . . . . . . . . . . . . . 11

3.3 A second-order accurate scheme and a high-resolution scheme . . . . . . . . . . 13

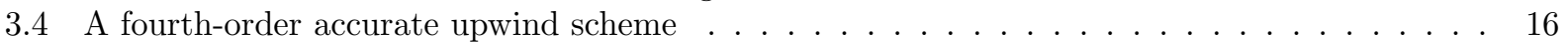

3.5 A sixth-order accurate upwind scheme $\ldots \ldots \ldots \ldots \ldots \ldots$

4 Upwind schemes in two space dimensions 19

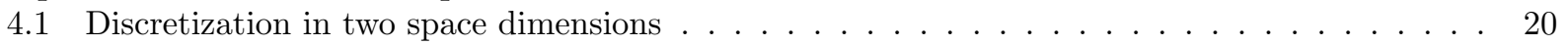

4.2 A first-order accurate scheme in two dimensions . . . . . . . . . . . . . . . . 22

4.3 A second-order accurate scheme and a high-resolution scheme in two dimensions . . . . . . 23

4.4 A fourth-order accurate scheme in two dimensions . . . . . . . . . . . . . . . 25

5 Accuracy, stability, points per wavelength and remarks $\quad 26$

${ }^{*}$ Corresponding author. Mailing address: Centre for Applied Scientific Computing, L-422, Lawrence Livermore National Laboratory, Livermore, CA 94551, USA. Phone: 925-423-2697. Fax: 925-424-2477.

Email addresses: banks20@llnl.gov (Jeffrey W. Banks), henshaw1@llnl.gov (William D. Henshaw)

${ }^{1}$ This work was performed under the auspices of the U.S. Department of Energy (DOE) by Lawrence Livermore National Laboratory under Contract DE-AC52-07NA27344 and by DOE contracts from the ASCR Applied Math Program. 


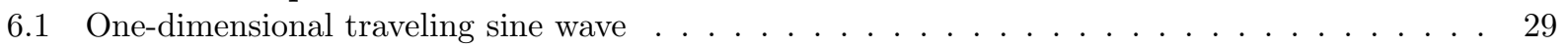

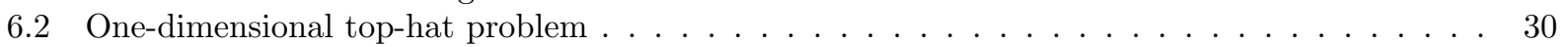

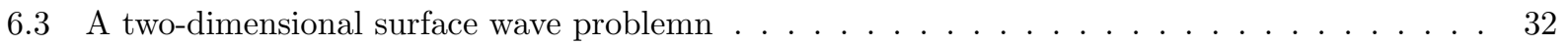

6.4 A top-hat problem in two space dimensions . . . . . . . . . . . . . . . . 34

\section{Conclusions}

\begin{tabular}{ll|l} 
Appendix A High-order accurate stencils & $\mathbf{3 7}$
\end{tabular}

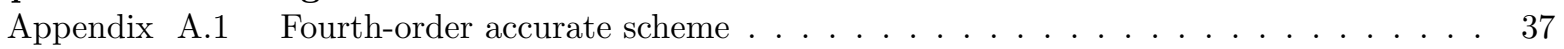

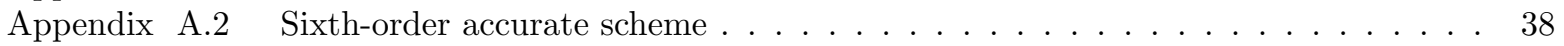

\section{Introduction}

Upwind methods for first-order systems of hyperbolic of partial differential equations (PDEs) have made a significant impact on computational science. They have facilitated the simulation of a wide variety of physical problems (e.g. fluid dynamics, solid mechanics, electromagnetics, plasma physics, as well as other wave propagation phenomena), using a wide variety of numerical methods (e.g. finite volume, finite difference, finite element, spectral element and discontinuous Galerkin). Upwind methods generally incorporate some aspects of the characteristic wave-structure of the hyperbolic system and thus include some of the attractive features of a method based on characteristics. An alternative approach to upwinding is based on the use of centered approximations and the explicit addition of artificial dissipation (artificial viscosity) or filters. This approach usually requires some tuning of the coefficients of the artificial dissipation and for difficult problems may require a matrix form of the dissipation that takes into account the characteristic structure $[1,2]$. Therefore, from one point of view, the success of upwind schemes is attributable to their ability to naturally add the appropriate amount and form of dissipation to the approximation by using the characteristic structure of the equations.

Upwinding was explicitly introduced by Godunov in his 1959 landmark paper [3]. In that work, a first-order accurate upwind method was devised for systems of hyperbolic partial differential equations in first-order form, including a treatment of the inviscid Euler equations. The key idea was to incorporate an exact solution of the Riemann problem into the numerical technique. Also recognized in that work was the inability for linear schemes to both maintain monotone profiles and at the same time achieve higher than first order accuracy for smooth flows (Godunov's theorem). Since the pioneering work of Godunov, there have been many extensions to the upwind approach including, for example, the flux-corrected transport method [4], the piecewise-parabolic-method (PPM) [5], essentially-non-oscillatory (ENO) schemes [6, 7], discontinuous Galerkin (DG) approximations [8], and the weighted-essentially-non-oscillatory (WENO) class of methods [9].

In this paper, we demonstrate how to construct high-order accurate upwind schemes for wave equations in second-order form without transforming the equations to a first-order system. This is, to our knowledge, the first systematic attempt to develop such schemes. The approach usually used to incorporate upwind discretizations into hyperbolic PDEs in second-order form is to transform the equations into a first-order system of equations. For example, the equations of linear elasticity, which take the form of a system of second-order hyperbolic PDEs for the displacements, can be transformed into a system of first-order hyperbolic PDEs for velocity and stress [10]. This is, of course, a valid and useful approach but there are a variety of reasons to consider solving the second-order PDEs directly.

One advantage of directly solving the equations in second-order form is that there are often significantly fewer dependent variables, and thus the schemes can be more efficient. For example, for Maxwell's equations of electromagnetism, the number of dependent variables can be reduced by a factor of two [11]. For the equations of linear elasticity in three dimensions, the second-order form has just three dependent variables (for the displacement) compared to between nine and fifteen for the first-order system (depending on which components of the displacement, velocity and stress are retained). Another advantage of solving the secondorder form of the equations is the removal of issues related to the equivalence, or lack thereof, between the first- and second-order systems. In general, first-order systems derived from second-order equations can admit a wider class of solutions and thus constraints must be imposed to ensure that solutions to the first-order system are also solutions to the second-order equations (e.g. the Saint-Venant compatibility

conditions for elasticity). For some systems, such as for the equations of general relativity, the number of constraint equations can become inordinately large which significantly complicates numerical approximation. 
In addition, many systems occur naturally as PDEs in second-order form and from a philosophical perspective there should be no particular reason why upwind style discretizations can't be derived in the native form. Finally we note that when solving problems in multiple space dimensions, it is often the case that a larger time-step can be taken for the second-order form of the equations compared to the first-order form [10].

An outline of the remainder of the manuscript follows. We begin, in Section 2 by developing a first-order accurate upwind scheme for the second-order wave equation that uses the d'Alembert solution to exactly advance a piecewise smooth representation of the solution. This geometric approach is analogous to Godunov's construction for advancing a piecewise constant representation of the solution to the first-order wave equation. In Section 3 we generalize this preliminary approach (which relies on a global representation of the exact solution) to a method that only requires the solution to local problems at cell faces. These localized problems are a generalization of the Riemann problem for the first-order system. This localized form is the key ingredient needed for extending the scheme to multiple space dimensions, high-order accuracy, variable coefficients and systems of equations (we leave variable coefficients and systems to future work). From the localized form we then derive high-order accurate space-time schemes using the Cauchy-Kowalewski procedure. We choose to develop conservative finite-difference approximations, although finite volume schemes are also possible. Schemes with orders of accuracy one, two, four and six are developed and analyzed using normal mode theory and modified equations. A second-order accurate high-resolution scheme (based on the use of nonlinear limiters) is also developed. The ideas are then generalized to two space dimensions in Section 4 where first-order accurate, second-order accurate, high-resolution and fourth-order accurate schemes are constructed and analyzed. The relationships between the modified equations, the solution error and the accuracy requirements in terms of points per wavelength is discussed in Section 5. Numerical examples are presented in Section 6. Smooth solutions in one space dimension (traveling sine wave) and two space dimensions (surface waves) are used to verify that the max-norm errors in the schemes converge at the expected rates. A top-hat problem, in both one and two space dimensions, is used to demonstrate the robust and high-quality approximations that result from the schemes when they are applied to difficult problems with discontinuous initial conditions. Section 7 provides concluding remarks while the stencil coefficients for the one-dimensional fourth- and sixth-order accurate schemes are given in appendices.

\section{Construction of an upwind scheme for the second-order wave equation in one di- mension}

Godunov's method and its extensions are well established for solving first-order systems of hyperbolic equations. These upwind-type methods use solutions to a set of Riemann problems defined at cell faces of a computational grid to construct a discrete approximation to the solution of a hyperbolic PDE. For linear problems, the Riemann solution is defined in terms of the characteristics of the hyperbolic operator. By using the characteristic variables, these methods naturally incorporate upwinding by tracing the influence of each characteristic variable along its respective space-time path.

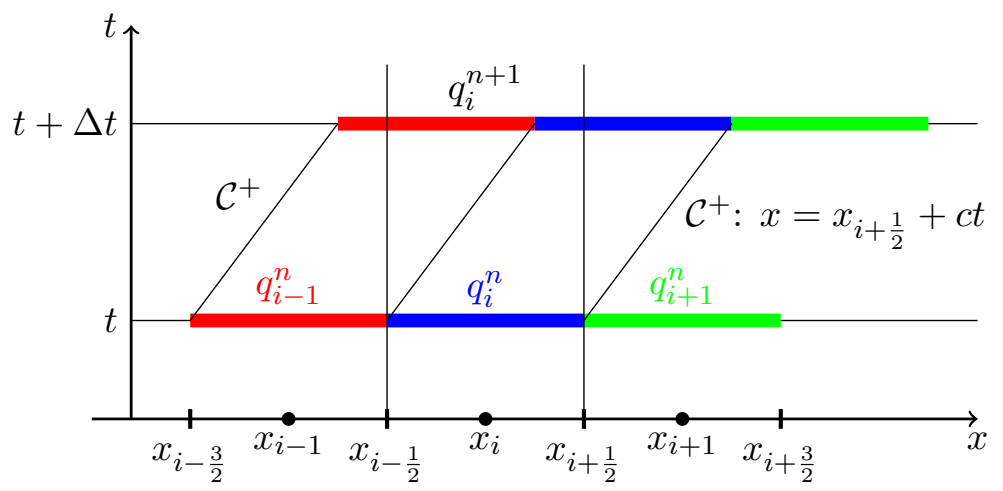

Figure 1: This figure illustrates the geometric construction of the first-order Godunov scheme for the first-order wave equation $q_{t}+c q_{x}=0$. The piecewise constant function that equals to $q_{i}^{n}$ on cell $\left(x_{i-\frac{1}{2}}, x_{i+\frac{1}{2}}\right)$ is exactly advanced to to time $t+\Delta t$. This is used to define discrete cell centered values, $q_{i}^{n+1}$, by integrating the solution over the cell. The resulting scheme is the standard first-order accurate upwind scheme.

It is useful to begin the discussion by reviewing a geometrically motivated derivation of a first-order 
accurate Godunov scheme for the initial value problem for the scalar first-order wave equation (FOWE),

$$
\begin{aligned}
& \frac{\partial q}{\partial t}+c \frac{\partial q}{\partial x}=0, \quad-\infty<x<\infty \\
& q(x, 0)=q_{0}(x) .
\end{aligned}
$$

Here $q=q(x, t)$ and for simplicity we assume that $c$ is constant with $c>0$. Introduce a uniform grid with cell centers $x_{i}=i h_{x}$ and cell faces $x_{i+\frac{1}{2}}=\left(i+\frac{1}{2}\right) h_{x}$, where $h_{x}>0$ is the grid spacing and $i=0, \pm 1 \pm 2, \ldots$ Let $q_{i}^{n} \approx q\left(x_{i}, t^{n}\right)$, be a grid function representing the solution at time $t^{n}=n \Delta t$, where $\Delta t$ is the time-step. At a given time $t^{n}$ we assume a known discrete solution at cell centers, $q_{i}^{n}$ and form the piecewise constant function

$$
Q_{0}(x)=q_{i}^{n}, \quad \text { for } x \in\left(x_{i-\frac{1}{2}}, x_{i+\frac{1}{2}}\right)
$$

For $\tau=t-t^{n}$, the exact solution to (1) with initial conditions $Q_{0}(x)$ can be found using the method of characteristics and is given by

$$
Q(x, \tau)=q_{i}^{n}, \quad \text { for } x-c \tau \in\left(x_{i-\frac{1}{2}}, x_{i+\frac{1}{2}}\right)
$$

The discrete cell-centered solution at the next time-step $t^{n+1}$ is defined as the cell average of $Q(x, \Delta t)$,

$$
q_{i}^{n+1}=\frac{1}{h_{x}} \int_{x_{i-\frac{1}{2}}}^{x_{i+\frac{1}{2}}} Q(x, \Delta t) d x .
$$

For time steps satisfying $\Delta t \leq h_{x} / c$ this construction is illustrated in Figure 1 and gives the standard first-order accurate upwind scheme,

$$
\begin{aligned}
q_{i}^{n+1} & =\frac{1}{h_{x}}\left[c \Delta t q_{i-1}^{n}+\left(h_{x}-c \Delta t\right) q_{i}^{n}\right], \\
& =q_{i}^{n}-\frac{c \Delta t}{h_{x}}\left(q_{i}^{n}-q_{i-1}^{n}\right) .
\end{aligned}
$$

Remark. Stability analysis reveals that the upwind scheme (2) is stable under the constraint $\Delta t \leq h_{x} / c$.

We now pursue a similarly intuitive construction applied to the one-dimensional second-order wave equation. Consider the initial value problem for the second-order wave equation (SOWE) in one-dimension,

$$
\begin{array}{r}
\frac{\partial^{2} u}{\partial t^{2}}=c^{2} \frac{\partial^{2} u}{\partial x^{2}}, \quad-\infty<x<\infty, \\
u(x, 0)=u_{0}(x), \quad \frac{\partial u}{\partial t}(x, 0)=v(x, 0)=v_{0}(x) .
\end{array}
$$

In analogy with elasticity, we call $u=u(x, t)$ the displacement and $v=\partial u / \partial t$ the velocity. An upwind method to solve the SOWE can be constructed as follows. Let $u_{i}^{n}$ and $v_{i}^{n}$, denote grid functions that are approximations to $u\left(x_{i}, t^{n}\right)$ and $v\left(x_{i}, t^{n}\right)$, respectively. Define a piecewise smooth representation of the discrete solution at time $t^{n}$ (as shown in Figure 2) by

$$
\begin{array}{ll}
U_{0}(x)=u_{i}^{n}+\frac{x-x_{i}}{h_{x}}\left(u_{i+1}^{n}-u_{i}^{n}\right), & \text { for } x \in\left(x_{i}, x_{i+1}\right), \\
V_{0}(x)=v_{i}^{n} & \text { for } x \in\left(x_{i-\frac{1}{2}}, x_{i+\frac{1}{2}}\right) .
\end{array}
$$

Here $U_{0}(x)$ is the continuous and piecewise linear function that passes through the values $\left(x_{i}, u_{i}^{n}\right)$. $V_{0}(x)$ is the piecewise constant function that passes through the points $\left(x_{i}, v_{i}^{n}\right)$ and is constant on cells $\left(x_{i-\frac{1}{2}}, x_{i+\frac{1}{2}}\right)$.

The exact solution corresponding to the initial conditions $U_{0}(x)$ and $V_{0}(x)$ can be determined from the well known d'Alembert solution to the second-order wave equation [12]. Letting $\tau=t-t^{n}$, this solution is given by

$$
\begin{aligned}
& U(x, \tau)=\frac{1}{2}\left(U_{0}(x+c \tau)+U_{0}(x-c \tau)\right)+\frac{1}{2 c} \int_{x-c \tau}^{x+c \tau} V_{0}(\xi) d \xi \\
& V(x, \tau)=\frac{\partial}{\partial t} U(x, \tau)=\frac{c}{2}\left(U_{0}^{\prime}(x+c \tau)-U_{0}^{\prime}(x-c \tau)\right)+\frac{1}{2}\left(V_{0}(x+c \tau)-V_{0}(x-c \tau)\right) .
\end{aligned}
$$




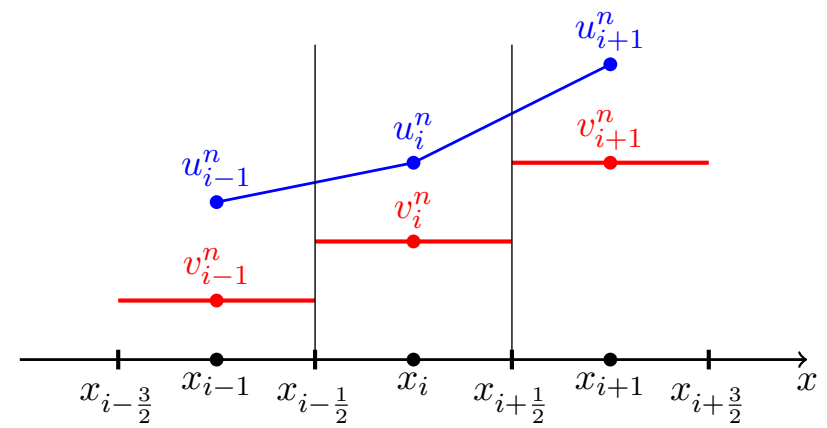

Figure 2: The approximate solution for the second-order wave equation in one-dimension is represented by the discrete grid functions $u_{i}^{n}$ (displacement) and $v_{i}^{n}$ (velocity) along with the corresponding piece-smooth functions shown in the figure.

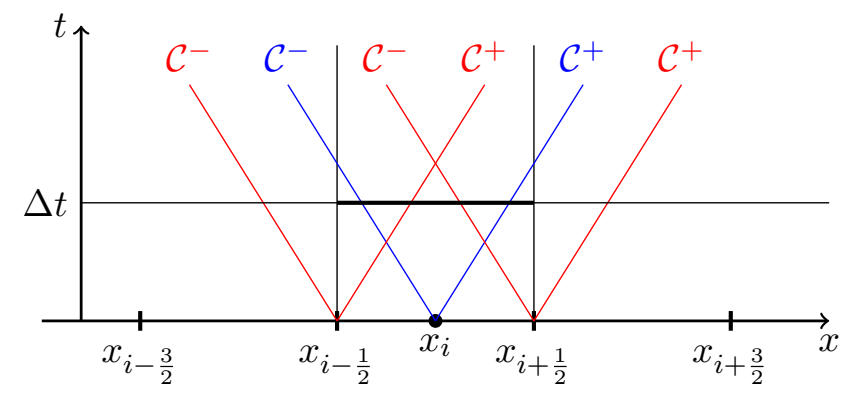

Figure 3: The $x-t$ diagram showing the characteristics emanating from the cell centers and cell faces.

As shown in Fig. 3, the form of this solution depends on the representation of $U_{0}(x)$ and $V_{0}(x)$ given in (3)-(4) and by the characteristics, $\mathcal{C}^{+}: d x / d t=c$, and $\mathcal{C}^{-}: d x / d t=-c$, that emanate from the points $x_{i \pm \frac{1}{2}}$ and $x_{i}$. To reconstruct the discrete solution at $t^{n+1}$ we compute cell averaged values of $U(x, \Delta t)$ and $V(x, \Delta t)$,

$$
u_{i}^{n+1}=\frac{1}{h_{x}} \int_{x_{i-\frac{1}{2}}}^{x_{i+\frac{1}{2}}} U(\xi, \Delta t) d \xi, \quad v_{i}^{n+1}=\frac{1}{h_{x}} \int_{x_{i-\frac{1}{2}}}^{x_{i+\frac{1}{2}}} V(\xi, \Delta t) d \xi .
$$

These integrals can be evaluated explicitly using the expressions (5)-(6) and the equations (3)-(4) for $U_{0}(x)$ and $V_{0}$. For $c \Delta t / h_{x}<\frac{1}{2}$ this procedure gives the following scheme, denoted by UW1a (for upwind scheme of order 1 , version a),

$$
\begin{aligned}
& u_{i}^{n+1}=u_{i}^{n}+\Delta t v_{i}^{n}+\left(\frac{c^{2} \Delta t^{2}}{2}+\frac{h_{x}^{2}}{8}\right) D_{+} D_{-} u_{i}^{n}+\frac{c \Delta t^{2}}{4} h_{x} D_{+} D_{-} v_{i}^{n}, \\
& v_{i}^{n+1}=v_{i}^{n}+c^{2} \Delta t D_{+} D_{-} u_{i}^{n}+\frac{c \Delta t}{2} h_{x} D_{+} D_{-} v_{i}^{n} .
\end{aligned}
$$

Here $D_{+}$and $D_{-}$are the usual forward and backward divided difference operators defined by $D_{+} w_{i}=$ $\left(w_{i+1}-w_{i}\right) / h_{x}$ and $D_{-} w_{i}=\left(w_{i}-w_{i-1}\right) / h_{x}$. For future reference we also note that the undivided forward and backward difference operators are defined by $\Delta_{+} w_{i}=w_{i+1}-w_{i}$ and $\Delta_{-} w_{i}=w_{i}-w_{i-1}$. Equations (7)-(8) define a first-order accurate upwind scheme for the one-dimensional second-order wave equation. A normal mode analysis shows that the scheme is stable provided $\Delta t \leq \Lambda_{1 a} h_{x} / c$ where $\Lambda_{1 a}=(1+\sqrt{5}) / 4 \approx .809$. The formal accuracy of this scheme can be determined by computing the truncation error. However, we proceed further and derive the so-called modified equations from which further properties of the discrete scheme can be gleaned. The modified equation is a continuous PDE whose solution describes the approximate behavior of the well resolved components of the discrete equations. The modified equation is derived by substituting continuous functions $U(x, t)$ and $V(x, t)$ into the discrete equations (7)-(8) by setting $u_{i}^{n}=U\left(x_{i}, t_{n}\right)$ and $v_{i}^{n}=V\left(x_{i}, t_{n}\right)$, and expanding all terms in Taylor series about the point $(x, t)=\left(x_{i}, t_{n}\right)$. This leads to the 
expansion

$$
\begin{aligned}
& \frac{\partial U}{\partial t}=V-\frac{\Delta t}{2} \frac{\partial^{2} U}{\partial t^{2}}+\frac{c^{2} \Delta t}{2} \frac{\partial^{2} U}{\partial x^{2}}-\frac{\Delta t^{2}}{6} \frac{\partial^{3} U}{\partial t^{3}}+\frac{c \Delta t h_{x}}{4} \frac{\partial^{2} V}{\partial x^{2}}+\frac{h_{x}^{2}}{8 \Delta t} \frac{\partial^{2} U}{\partial x^{2}}+\mathcal{O}\left(\left(\Delta t+h_{x}\right)^{3}\right), \\
& \frac{\partial V}{\partial t}=c^{2} \frac{\partial^{2} U}{\partial x^{2}}-\frac{\Delta t}{2} \frac{\partial^{2} V}{\partial t^{2}}+\frac{c h_{x}}{2} \frac{\partial^{2} V}{\partial x^{2}}-\frac{\Delta t^{2}}{6} \frac{\partial^{3} V}{\partial t^{3}}+c^{2} \frac{h_{x}^{2}}{12} \frac{\partial^{4} U}{\partial x^{4}}+\mathcal{O}\left(\left(\Delta t+h_{x}\right)^{3}\right) .
\end{aligned}
$$

The time derivatives on the right hand sides of (9)-(10) can be eliminated by differentiating (9)-(10) with respect to time and recursively substituting the result back into the right hand sides of (9)-(10). This leads to the following modified equations satisfied by $U$ and $V$

$$
\begin{aligned}
\frac{\partial^{2} U}{\partial t^{2}} & =c^{2} \frac{\partial^{2} U}{\partial x^{2}}+\frac{c h_{x}}{8 \lambda}\left(\left(-4 \lambda^{2}+4 \lambda+1\right) \frac{\partial^{3} U}{\partial t \partial x^{2}}\right)+\mathcal{O}\left(\left(\Delta t+h_{x}\right)^{2}\right), \\
\frac{\partial^{2} V}{\partial t^{2}} & =c^{2} \frac{\partial^{2} V}{\partial x^{2}}+\frac{c h_{x}}{8 \lambda}\left(\left(-4 \lambda^{2}+4 \lambda+1\right) \frac{\partial^{3} V}{\partial t \partial x^{2}}\right)+\mathcal{O}\left(\left(\Delta t+h_{x}\right)^{2}\right),
\end{aligned}
$$

where $\lambda=c \Delta t / h_{x}$ is the CFL parameter. From the modified equations (11)-(12) we see the scheme is formally first-order accurate since the first correction term is $\mathcal{O}\left(h_{x}\right)$. Note that this correction term, (the term involving $\partial^{3} U / \partial t \partial x^{2}$ for the $U$ equation (11)), is dissipative in nature, and is a reflection of the upwind approximation.

The behavior of the upwind scheme scheme (7)-(8) is illustrated for a particularly difficult problem. The initial conditions consist of a top-hat function for the displacement and zero initial velocity,

$$
\begin{aligned}
& u(x, 0)=H\left(x+\frac{1}{4}\right)-H\left(x-\frac{1}{4}\right), \\
& v(x, 0)=0 .
\end{aligned}
$$

Here $H(x)$ is the Heaviside function defined by $H(x)=0$ for $x<0$ and $H(x)=1$ for $x \geq 0$. The exact solution can be found by appealing to d'Alembert's solution and is given as

$$
\begin{aligned}
& u(x, t)=\frac{1}{2}\left[H\left(x+c t+\frac{1}{4}\right)-H\left(x+c t-\frac{1}{4}\right)+H\left(x-c t+\frac{1}{4}\right)-H\left(x-c t-\frac{1}{4}\right)\right], \\
& v(x, t)=\frac{c}{2}\left[\delta\left(x+c t+\frac{1}{4}\right)-\delta\left(x+c t-\frac{1}{4}\right)-\delta\left(x-c t+\frac{1}{4}\right)+\delta\left(x-c t-\frac{1}{4}\right)\right] .
\end{aligned}
$$

Solving this problem numerically is difficult since the solution for $u$ does not have a continuous first derivative, while $v$ is defined in terms of Dirac delta functions. Figure 4 shows the discrete solution for the displacement and velocity at a number of times, computed using the scheme (7)-(8). For this computation we took $c=1$ and used $N=201$ grid points on the interval $[-1,1]$ with $h_{x}=2 /(N-1)$. The time-step $\Delta t$ was chosen to be a factor of 0.9 of the maximum allowed by stability.

For comparison we show, in Figure 5, results from the UW1a scheme, results from the UW1 scheme (47)(48) (which is derived in Section 3.2 using a more general procedure), and results computed using the standard centered finite difference scheme to the SOWE given by

$$
\frac{u_{i}^{n+1}-2 u_{i}^{n}+u_{i}^{n-1}}{\Delta t^{2}}=c^{2} D_{+} D_{-} u_{i}^{n} .
$$

We denote the scheme (17) as the C2 scheme (for centered scheme of second-order accuracy). The velocity for the $\mathrm{C} 2$ scheme, as shown in this figure, is a derived quantity computed using a centered second-order finite difference operator in time as $v_{i}^{n}=\frac{1}{2 \Delta t}\left(u_{i}^{n+1}-u_{i}^{n-1}\right)$. Notice the qualitative differences between the standard centered approximation to the new upwind approximations. The centered scheme displays the usual highly oscillatory phenomena near discontinuities. The upwind schemes are much smoother near the discontinuities with scheme UW1 having a sharper resolution than UW1a.

Remark: Some care must be taken in imposing initial conditions for the $\mathrm{C} 2$ scheme. For example if the exact solution at $t=0$ and $t=\Delta t$ are used, convergence to the intended weak solution is not guaranteed even in an $L_{1}$ sense. The approximation presented in Figure 5 was obtained by applying an RK-2 procedure from $t=0$ to $t=\Delta t$ and then moving to the $\mathrm{C} 2$ scheme for subsequent time-stepping. 

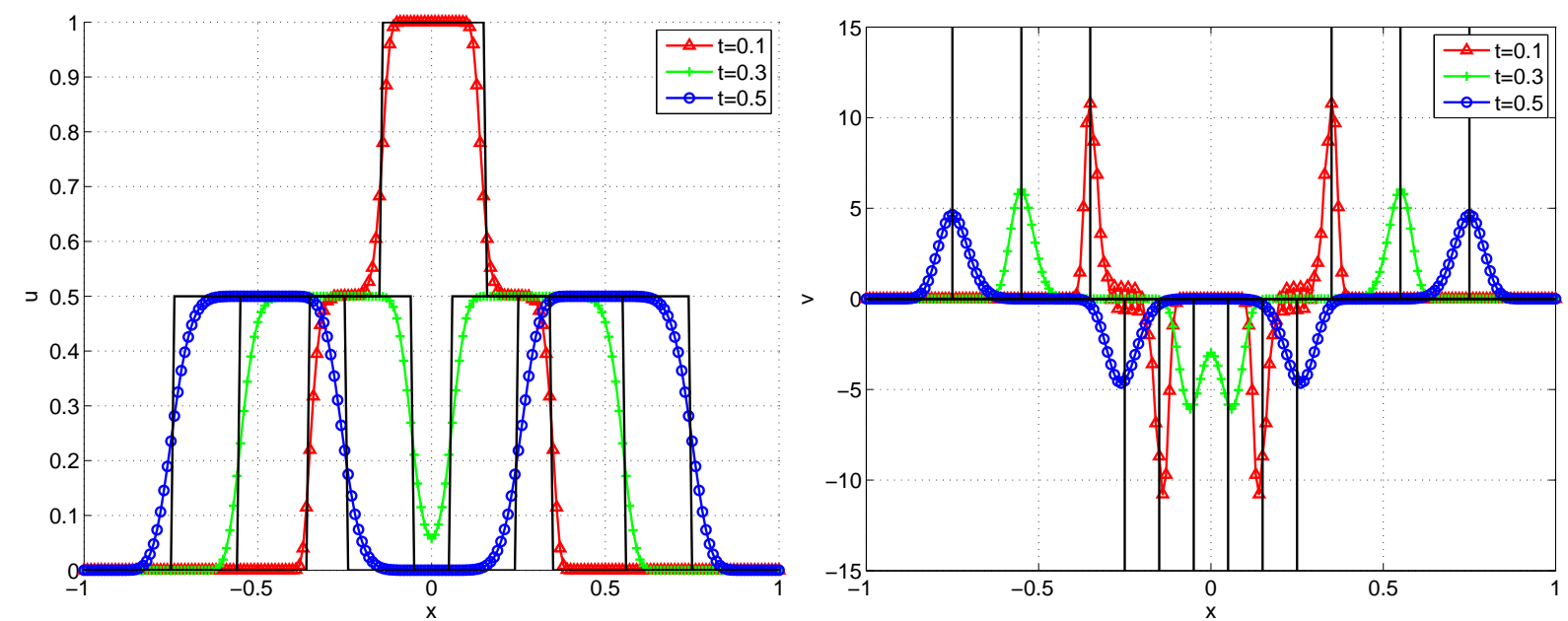

Figure 4: Results for the top-hat initial condition of Section 6.2 using the preliminary first-order accurate upwind scheme UW1a. Results are shown at $t=0.1,0.3$ and 0.5 for $u$ (left) and $v$ (right). Notice that the location of the Dirac delta functions in the exact solution for $v$ are indicated by vertical black lines.
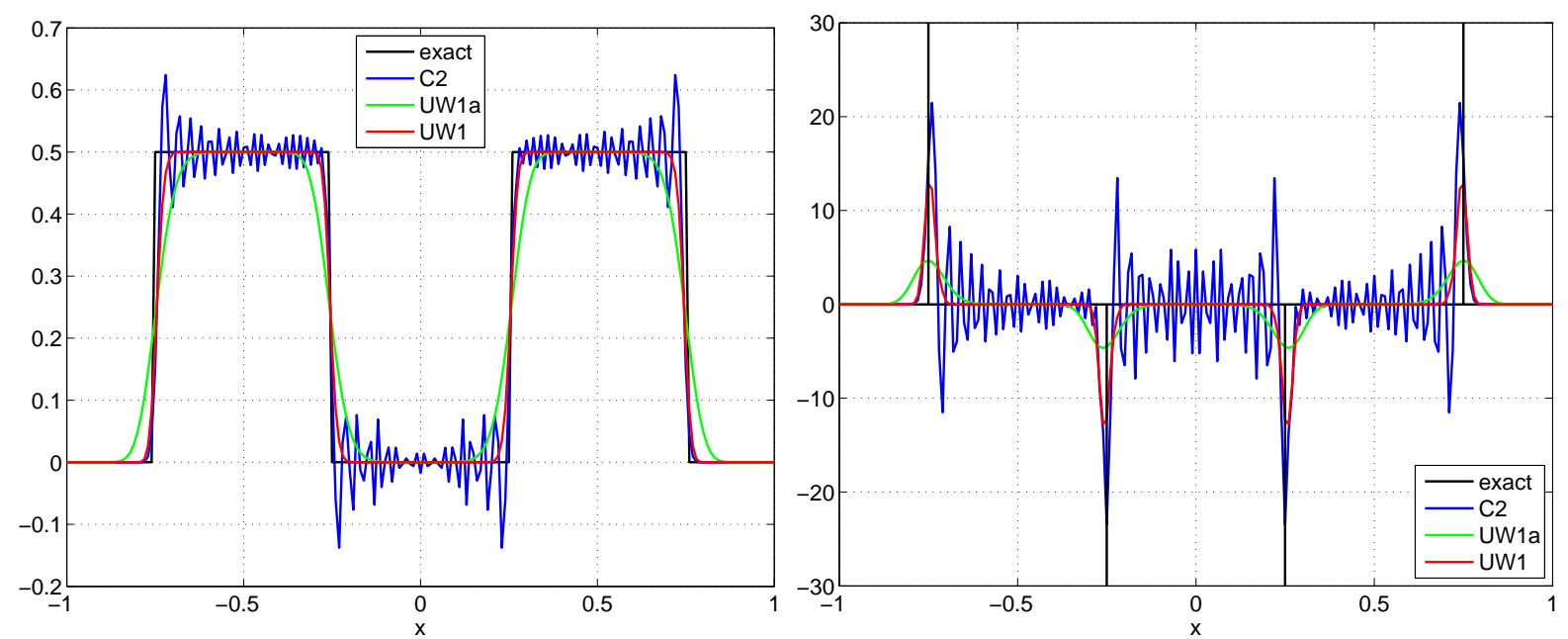

Figure 5: Results for the top hat initial condition of Section 6.2 using the preliminary first-order scheme UW1a, the C2 scheme, and the alternative first-order upwind scheme UW1 (47)-(48). Left: displacements $u$. Right: velocity $v$ (note the vertical scale). The exact (weak) solution for the velocity is given by four traveling delta functions which are displayed as vertical black lines.

Remark. The scheme of (7) and (8) is conservative since, letting $\mathcal{S}\left(f_{i}\right)=\sum_{i=\mu}^{\nu} f_{i} h_{x}$ denote a discrete approximation to the integral, then

$$
\begin{aligned}
& \mathcal{S}\left(u_{i}^{n+1}\right)=\mathcal{S}\left(u_{i}^{n}\right)+\Delta t \mathcal{S}\left(v_{i}^{n}\right) h_{x}+\text { boundary terms }, \\
& \mathcal{S}\left(v_{i}^{n+1}\right)=\mathcal{S}\left(v_{i}^{n}\right)+\text { boundary terms. }
\end{aligned}
$$

Remark. The step of forming the piecewise polynomial solution $U_{0}(x)$ and $V_{0}(x)$ in (3)-(4) from the grid functions $u_{i}^{n}$ and $v_{i}^{n}$ is often called the reconstruction step [13]. There are many choices for this reconstruction and we present just one. This one has the property that the d'Alembert solution given by (5)-(6) has the same degree of smoothness as the initial conditions; i.e. $U(x, t)$ remains continuous and piecewise linear and $V(x, t)$ remains piecewise constant. In contrast, choosing a piecewise constant reconstruction for $U_{0}(x)$, would result in a d'Alembert solution with Dirac delta functions in $V(x, t)$. It seems wise to avoid such a singular solution in our discrete representation. In addition, for consistency it seems appropriate to choose a reconstruction for $U_{0}(x)$ that is one derivative smoother than the reconstruction for $V_{0}(x)$. This is consistent with the fact that $v$ is a time derivative of $u$ and that for the wave equation the time derivative has the same degree of smoothness as a space derivative. 


\section{A general construction for high-order upwind schemes - the one-dimensional case}

We now consider a more general development of upwind schemes for the SOWE. This construction is similar in nature to the construction of upwind methods for the first-order system using solutions to Riemann problems at cell faces rather than direct integration of exact solutions as presented in Section 2. Consider the initial value problem for second order wave equation in one space dimension,

$$
\begin{aligned}
\frac{\partial^{2} u}{\partial t^{2}} & =L u, \quad x \in \Omega, \\
L & \equiv c^{2} \frac{\partial^{2}}{\partial x^{2}}, \\
u(x, 0) & =u_{0}(x), \quad u_{t}(x, 0)=v_{0}(x),
\end{aligned}
$$

where $u=u(x, t)$ is taken to be periodic in $x$ on the interval $\Omega=[0,1]$ and where $c$ is constant with $c>0$. We write (18) in the form of a conservation law,

$$
\left[\begin{array}{l}
u \\
v
\end{array}\right]_{t}=\left[\begin{array}{c}
0 \\
c^{2} u_{x}
\end{array}\right]_{x}+\left[\begin{array}{l}
v \\
0
\end{array}\right]
$$

Introduce a uniform Cartesian grid for $\Omega$ with $N+1$ grid points. Let $h_{x}=1 / N$ denote the grid spacing. Denote the grid cell centers by $x_{i}=\left(i+\frac{1}{2}\right) h_{x}, i=0,1, \ldots, N-1$ and the grid vertices by $x_{i-\frac{1}{2}}=i h_{x}$, $i=0,1, \ldots, N$. We choose to pursue a so-called conservative finite-difference approach by discretizing the time integrated equations. One could alternately follow a finite-volume approach and integrate the equations over a space-time cell. This choice is largely one of personal preference, and the mechanics of developing finite-volume schemes should be clear from the following discussion.

The derivation of the scheme begins by integrating the equation for the velocity in time,

$$
v(x, t)=v(x, 0)+c^{2} \int_{0}^{t} \frac{\partial^{2} u}{\partial x^{2}}(x, \tau) d \tau .
$$

This expression can be transformed into a form that resembles a discrete conservation approximation by first writing $\partial^{2} u / \partial x^{2}$ as the forward divided difference of the face centered flux function $f\left(x-\frac{h_{x}}{2}, t\right)$,

$$
\begin{aligned}
\frac{\partial^{2} u}{\partial x^{2}}(x, t) & =D_{+} f\left(x-\frac{h_{x}}{2}, t\right), \\
f(x, t) & \equiv \mathcal{D}_{x} \frac{\partial u}{\partial x}(x, t),
\end{aligned}
$$

where the application of $D_{+}$to continuous functions is defined to be $D_{+} f(x, t)=\left(f\left(x+h_{x}, t\right)-f(x, t)\right) / h_{x}$. The differential-difference operator $\mathcal{D}_{x}$ is defined to satisfy the identity

$$
\frac{\partial w}{\partial x}(x)=D_{+}\left(\mathcal{D}_{x} w\left(x-\frac{h_{x}}{2}\right)\right),
$$

for any sufficiently smooth function $w$ and is formally given by the expansion

$$
\begin{aligned}
\mathcal{D}_{x} w(x, t) & =\sum_{j=0}^{\infty} \alpha_{j} h_{x}^{2 j} \frac{\partial^{2 j} w}{\partial x^{2 j}}(x, t), \\
& =w(x, t)-\frac{h_{x}^{2}}{24} \frac{\partial^{2} w}{\partial x^{2}}(x, t)+\frac{7 h_{x}^{4}}{5760} \frac{\partial^{4} w}{\partial x^{4}}(x, t)-\frac{31 h_{x}^{6}}{967680} \frac{\partial^{6} w}{\partial x^{6}}(x, t)+\ldots .
\end{aligned}
$$

The coefficients $\alpha_{j}$ can be computed from the identity $2 \zeta=\sinh (\zeta / 2) \sum_{j=0}^{\infty} \alpha_{j} \zeta^{2 j}$. Using (20) in (19) gives the following equation for $v(x, t)$

$$
v(x, t)=v(x, 0)+c^{2} D_{+}\left[\int_{0}^{t} f\left(x-\frac{h_{x}}{2}, \tau\right) d \tau\right] .
$$

This equation is integrated in time to give an exact equation for $u(x, t)$,

$$
u(x, t)=u(x, 0)+t v(x, 0)+c^{2} D_{+}\left[\int_{0}^{t} \int_{0}^{\tau} f\left(x-\frac{h_{x}}{2}, \tau^{\prime}\right) d \tau^{\prime} d \tau\right] .
$$


Taking these integrations over a single time-step leads to the following conservation form for the solution at time $t^{n+1}$

$$
\begin{aligned}
v\left(x, t^{n+1}\right) & =v\left(x, t^{n}\right)+c^{2} \Delta t D_{+}\left[\mathcal{F}^{v}\left(x-\frac{h_{x}}{2}, t^{n}\right)\right] \\
u\left(x, t^{n+1}\right) & =u\left(x, t^{n}\right)+\Delta t v\left(x, t^{n}\right)+c^{2} \Delta t^{2} D_{+}\left[\mathcal{F}^{u}\left(x-\frac{h_{x}}{2}, t^{n}\right)\right], \\
\mathcal{F}^{v}\left(x, t^{n}, \Delta t\right) & \equiv \frac{1}{\Delta t} \int_{0}^{\Delta t} f\left(x, t^{n}+\tau\right) d \tau \\
\mathcal{F}^{u}\left(x, t^{n}, \Delta t\right) & \equiv \frac{1}{\Delta t^{2}} \int_{0}^{\Delta t} \int_{0}^{\tau} f\left(x, t^{n}+\tau^{\prime}\right) d \tau^{\prime} d \tau .
\end{aligned}
$$

We emphasize that (24)-(27) is a formally exact differential-difference equation for the solution. Different numerical schemes are developed by considering various upwind approximations to $f\left(x-h_{x} / 2, t^{n}+\tau\right)$ in $(26)$ (27).

\subsection{The upwind flux for the second-order system}

One way to incorporate upwinding into the scheme (24)-(27) is to use $x$-derivatives of the d'Alembert solution (15) to derive expressions for derivatives of $u$ on the face in terms of the solution at the previous time step $t^{n}$

$$
\begin{aligned}
\frac{\partial^{p}}{\partial x^{p}} u\left(x, t^{n}+\tau\right) & \left.=\frac{1}{2}\left(\frac{\partial^{p}}{\partial x^{p}} u(x+c \tau), t^{n}\right)+\frac{\partial^{p}}{\partial x^{p}} u\left(x-c \tau, t^{n}\right)\right) \\
& +\frac{1}{2 c}\left[\frac{\partial^{p-1}}{\partial x^{p-1}} v\left(x+c \tau, t^{n}\right)-\frac{\partial^{p-1}}{\partial x^{p-1}} v\left(x-c \tau, t^{n}\right)\right], \quad \text { for } p=1,2, \ldots
\end{aligned}
$$

Use of (28) in (24)-(25) could be used as a starting point for a characteristic-based upwind scheme for the SOWE. We do not, however, follow this line of approximation here. Instead, we use another approach to incorporating upwinding that can be more easily incorporated into conservative finite difference approximations. This alternative approach makes use of fact that taking the limit $\tau \rightarrow 0$ in (28) gives

$$
\begin{aligned}
\frac{\partial^{p}}{\partial x^{p}} u(x, t+) & =\frac{1}{2}\left(\frac{\partial^{p}}{\partial x^{p}} u(x+, t)+\frac{\partial^{p}}{\partial x^{p}} u(x-, t)\right) \\
& +\frac{1}{2 c}\left[\frac{\partial^{p-1}}{\partial x^{p-1}} v(x+, t)-\frac{\partial^{p-1}}{\partial x^{p-1}} v(x-, t)\right], \quad \text { for } p=1,2, \ldots
\end{aligned}
$$

Here $t+$ denotes the limit of $t+\epsilon$ as $\epsilon \rightarrow 0$ with $\epsilon>0$. Similarly, $x+$ and $x$ - denote values of $x$ infinitesimally larger and smaller than $x$, respectively. Formula (29) is the key result that we will use to derive upwind schemes to any order of accuracy. It specifies how to define an upwind biased flux on a face, given left and right approximations to the spatial derivatives of the displacement and velocity on either side of the face. In particular, the upwind flux approximation for the $p^{\text {th }} x$-derivative of $u$ is the average of the $p^{\text {th }}$ $x$-derivative of the left and right displacement states, plus the reciprocal of $2 c$ times the difference of the $(p-1)^{\text {st }} x$-derivative of the left and right velocity states.

Our assumed form of the discrete solution for a low order approximation was previously shown in Figure 2 and consists of a piece-wise linear representation for $u$ and a piecewise constant representation for $v$. For this form we note that the first derivative of $u$ is continuous at the cell faces while $v$ may jump. With this assumed form we can simplify $(29)$ by replacing the average of the left and right approximations to $\frac{\partial^{p}}{\partial x^{p}} u(x, t)$ in (29) with a centered approximation,

$$
\frac{\partial^{p}}{\partial x^{p}} u(x, t+)=\frac{\partial^{p}}{\partial x^{p}} u(x, t)+\frac{1}{2 c}\left[\frac{\partial^{p-1}}{\partial x^{p-1}} v(x+, t)-\frac{\partial^{p-1}}{\partial x^{p-1}} v(x-, t)\right], \quad \text { for } p=1,2, \ldots
$$

This centered approximation to $\partial_{x}^{p} u(x, t)$ also leads to more compact difference approximations, which are generally preferable for stability and accuracy reasons.

Definition The upwind flux function $\check{f}$ is defined to be

$$
\check{f}\left(x+\frac{h_{x}}{2}, t^{n}+\tau\right) \equiv \mathcal{D}_{x} \frac{\partial u}{\partial x}\left(x_{i+\frac{1}{2}}, t^{n}+\tau\right)+\frac{1}{2 c}\left[\mathcal{D}_{x} v^{+}\left(x_{i+\frac{1}{2}}, t^{n}+\tau\right)-\mathcal{D}_{x} v^{-}\left(x_{i+\frac{1}{2}}, t^{n}+\tau\right)\right],
$$

where $v^{-}$and $v^{+}$denote left and right limits, respectively, to $v$ on the face. 
The upwind flux function (31) replaces $f$ in the scheme (24)-(25) to define an upwind scheme. Upwind schemes with different orders of accuracy are determined by approximating terms in (31) with the appropriate accuracy. The Cauchy-Kowalewski process, also known as the Lax-Wendroff procedure [14], is used to develop space-time schemes using data from only two time levels. In this approach, equation (31) is expanded using Taylor series in space and time, and the governing equations are used to replace temporal by spatial derivatives. Specifically, Taylor expansions of $\partial u / \partial x$ about the face position $x_{i+\frac{1}{2}}$ and $v$ about the cell center $x_{i}$ give

$$
\begin{aligned}
\frac{\partial u}{\partial x}\left(x_{i+\frac{1}{2}}, t^{n}+\tau\right) & =\sum_{m=0}^{\infty} \frac{\tau^{m}}{m !} \frac{\partial^{m}}{\partial t^{m}} \frac{\partial u}{\partial x}\left(x_{i+\frac{1}{2}}, t^{n}\right) \\
v\left(x_{i} \pm \frac{h_{x}}{2}, t^{n}+\tau\right) & =\sum_{m=0}^{\infty} \sum_{n=0}^{m}\left(\begin{array}{c}
m \\
n
\end{array}\right) \frac{1}{m !} \tau^{m-n}\left(\frac{ \pm h_{x}}{2}\right)^{n} \frac{\partial^{m} v\left(x_{i}, t^{n}\right)}{\partial t^{m-n} \partial x^{n}}
\end{aligned}
$$

From (33), left and right biased expressions for the velocity on the face are given as

$$
\begin{aligned}
& v^{-}\left(x_{i+\frac{1}{2}}, t^{n}+\tau\right) \equiv v\left(x_{i}+\frac{h_{x}}{2}, t^{n}+\tau\right), \\
& v^{+}\left(x_{i+\frac{1}{2}}, t^{n}+\tau\right) \equiv v\left(x_{i+1}-\frac{h_{x}}{2}, t^{n}+\tau\right) .
\end{aligned}
$$

Note that the expansion determining $v^{-}$originates from $x_{i}$, while that for $v^{+}$originates from $x_{i+1}$. Time derivatives can be converted to space derivatives using the governing equation $u_{t t}=L u$. Letting,

$$
\mathcal{L}_{m}(u, v) \equiv \begin{cases}L^{m / 2} u & \text { if } m \text { is even } \\ L^{(m-1) / 2} v & \text { if } m \text { is odd }\end{cases}
$$

it follows that $\partial_{t}^{m} u=\mathcal{L}_{m}(u, v)$ and $\partial_{t}^{m} v=\mathcal{L}_{m+1}(v, u)$. Using (32)-(33) together with (23) and definitions (34) and (35) in (31) gives,

Proposition 3.1. The space-time expansion for the upwind flux (31) is given by

$$
\begin{aligned}
& \check{f}\left(x_{i+\frac{1}{2}}, t^{n}+\tau\right)=\sum_{j=0}^{\infty} \sum_{m=0}^{\infty} \alpha_{j} h_{x}^{2 j}\left\{\frac{\tau^{m}}{m !} \frac{\partial^{2 j+1}}{\partial x^{2 j+1}} \mathcal{L}_{m}(u, v)\left(x_{i+\frac{1}{2}}, t^{n}\right)\right. \\
& \left.\quad+\frac{1}{2 c} \sum_{n=0}^{m}\left(\begin{array}{c}
m \\
n
\end{array}\right) \frac{\tau^{m-n}}{m !}\left(\frac{h_{x}}{2}\right)^{n} \frac{\partial^{n+2 j}}{\partial x^{n+2 j}}\left[(-1)^{n} \mathcal{L}_{m-n+1}(v, u)\left(x_{i+1}, t^{n}\right)-\mathcal{L}_{m-n+1}(v, u)\left(x_{i}, t^{n}\right)\right]\right\} .
\end{aligned}
$$

The single and double time integrals of the expansion for the upwind flux (37) required for (26)-(26) can be easily obtained, which leads to,

Proposition 3.2. Upwind approximations to the time averaged flux functions (26)-(26) are given by

$$
\begin{aligned}
& \mathcal{F}^{v}\left(x_{i+\frac{1}{2}}, t^{n}, \Delta t\right) \approx \mathcal{F}_{i+\frac{1}{2}}^{v}\left(t^{n}, \Delta t\right)=F(\Delta t,\{1 /(p+1)\}) \\
& \mathcal{F}^{u}\left(x_{i+\frac{1}{2}}, t^{n}, \Delta t\right) \approx \mathcal{F}_{i+\frac{1}{2}}^{u}\left(t^{n}, \Delta t\right)=F(\Delta t,\{1 /((p+1)(p+2))\})
\end{aligned}
$$

where $\left\{k_{p}\right\}$ denotes the set $\left\{k_{p}\right\}_{p=0}^{\infty}$, and

$$
\begin{aligned}
& F\left(\tau,\left\{k_{p}\right\}\right)=\sum_{j=0}^{\infty} \sum_{m=0}^{\infty} \alpha_{j} h_{x}^{2 j}\left\{\frac{k_{m} \tau^{m}}{m !} \frac{\partial^{2 j+1}}{\partial x^{2 j+1}} \mathcal{L}_{m}(u, v)\left(x_{i+\frac{1}{2}}, t^{n}\right)\right. \\
& \left.\quad+\frac{1}{2 c} \sum_{n=0}^{m}\left(\begin{array}{c}
m \\
n
\end{array}\right) \frac{k_{m-n} \tau^{m-n}}{m !}\left(\frac{h_{x}}{2}\right)^{n} \frac{\partial^{n+2 j}}{\partial x^{n+2 j}}\left[(-1)^{n} \mathcal{L}_{m-n+1}(v, u)\left(x_{i+1}, t^{n}\right)-\mathcal{L}_{m-n+1}(v, u)\left(x_{i}, t^{n}\right)\right]\right\} .
\end{aligned}
$$


Letting $L=c^{2} \partial_{x}^{2}$, the first few terms in the expansion for $F\left(\tau,\left\{k_{p}\right\}\right)$ are given by

$$
\begin{aligned}
F\left(\tau,\left\{k_{p}\right\}\right) & =k_{0} \frac{\partial u}{\partial x}\left(x_{i+\frac{1}{2}}, t^{n}\right)+\frac{k_{0}}{2 c} \Delta_{+} v\left(x_{i}, t^{n}\right) \\
& +k_{1} \tau \frac{\partial v}{\partial x}\left(x_{i+\frac{1}{2}}, t^{n}\right)+\frac{k_{1} c \tau}{2} \Delta_{+} \frac{\partial^{2} u}{\partial x^{2}}\left(x_{i}, t^{n}\right)-\frac{k_{0} h_{x}}{2 c} \mathcal{A}_{+} \frac{\partial v}{\partial x}\left(x_{i}, t^{n}\right) \\
& +\left(\frac{k_{2} c^{2} \tau^{2}}{2}-\frac{k_{0} h_{x}^{2}}{24}\right) \frac{\partial^{3} u}{\partial x^{3}}\left(x_{i+\frac{1}{2}}, t^{n}\right)+\left(\frac{k_{2} c \tau^{2}}{4}+\frac{k_{0} h_{x}^{2}}{24 c}\right) \Delta_{+} \frac{\partial^{2} v}{\partial x^{2}}\left(x_{i}, t^{n}\right)-\frac{k_{1} c \tau h_{x}}{2} \mathcal{A}_{+} \frac{\partial^{3} u}{\partial x^{3}}\left(x_{i}, t^{n}\right) \\
& +\left(\frac{k_{3} c^{2} \tau^{3}}{6}-\frac{k_{1} \tau h_{x}^{2}}{24}\right) \frac{\partial^{3} v}{\partial x^{3}}\left(x_{i+\frac{1}{2}}, t^{n}\right)+\left(\frac{k_{3} c^{3} \tau^{3}}{12}+\frac{k_{1} c \tau h_{x}^{2}}{24}\right) \Delta_{+} \frac{\partial^{4} u}{\partial x^{4}}\left(x_{i}, t^{n}\right) \\
& -\frac{k_{2} c \tau^{2} h_{x}}{4} \mathcal{A}_{+} \frac{\partial^{3} v}{\partial x^{3}}\left(x_{i}, t^{n}\right)+\mathcal{O}\left(\left(h_{x}+\Delta t\right)^{4}\right) .
\end{aligned}
$$

Here $\mathcal{A}_{+}$is the forward averaging operator, $\mathcal{A}_{+} u\left(x_{i}, t^{n}\right)=\frac{1}{2}\left(u\left(x_{i+1}, t^{n}\right)+u\left(x_{i}, t^{n}\right)\right)$.

Remark. There is a choice in approximating $\partial v\left(x_{i}, t^{n}\right) / \partial x, \partial^{3} v\left(x_{i}, t^{n}\right) / \partial x^{3}$ and $\partial^{3} u\left(x_{i}, t^{n}\right) / \partial x^{3}$ in (41) since these terms are not centered properly. As a result, it is natural to consider limiting these terms.

From an implementation point of view it may be more efficient to use a quadrature rule, rather than integrating exactly in time. In particular, if one uses Gaussian quadrature with quadrature points $\xi_{j}$, $j=1,2, \ldots, M$ for the interval $[0,1]$, then approximations to both $\mathcal{F}^{v}\left(x_{i+\frac{1}{2}}, t^{n}, \Delta t\right)$ and $\mathcal{F}^{u}\left(x_{i+\frac{1}{2}}, t^{n}, \Delta t\right)$ can be computed from $M$ evaluations of an approximation to the flux function (41)

$$
\begin{aligned}
F_{i+\frac{1}{2}}^{j} & \approx F\left(\xi_{j} \Delta t,\{1\}\right), \quad j=1,2, \ldots, M, \\
\mathcal{F}^{v}\left(x_{i+\frac{1}{2}}, t^{n}, \Delta t\right) & \approx \mathcal{F}_{i+\frac{1}{2}}^{v}\left(t^{n}, \Delta t\right)=\sum_{j=1}^{M} b_{j} F_{i+\frac{1}{2}}^{j}, \\
\mathcal{F}^{u}\left(x_{i+\frac{1}{2}}, t^{n}, \Delta t\right) & \approx \mathcal{F}_{i+\frac{1}{2}}^{u}\left(t^{n}, \Delta t\right)=\sum_{j=1}^{M} d_{j} F_{i+\frac{1}{2}}^{j} .
\end{aligned}
$$

Here the weights $b_{j}$ and $d_{j}$ are given by the integrals of the interpolation basis function $\phi_{j}(\tau)$,

$$
\phi_{j}(\tau)=\frac{\prod_{k=1, k \neq j}^{M}\left(\tau-\xi_{k}\right)}{\prod_{k=1, k \neq j}^{M}\left(\xi_{j}-\xi_{k}\right)}, \quad b_{j}=\int_{0}^{1} \phi_{j}(\tau) d \tau, \quad d_{j}=\int_{0}^{1} \int_{0}^{\tau^{\prime}} \phi_{j}\left(\tau^{\prime}\right) d \tau^{\prime} d \tau .
$$

Note that for the special case $M=1, \phi_{1}(\tau)=1$. Since Gaussian quadrature is exact for polynomials up to degree $2 M-1$ and accurate to $\mathcal{O}\left(\Delta t^{2 M}\right)$, we can choose $M=q$ for an approximation of order $2 q, q=1,2, \ldots$.

Upwind approximations of different orders of accuracy can now be defined by approximating the terms in (41) to different orders of accuracy and substituting into

$$
\begin{aligned}
& v_{i}^{n+1}=v_{i}^{n}+c^{2} \Delta t D_{+}\left[\mathcal{F}_{i-\frac{1}{2}}^{v}\left(t^{n}, \Delta t\right)\right], \\
& u_{i}^{n+1}=u_{i}^{n}+\Delta t v_{i}^{n}+c^{2} \Delta t^{2} D_{+}\left[\mathcal{F}_{i-\frac{1}{2}}^{u}\left(t^{n}\right)\right] .
\end{aligned}
$$

\subsection{A first-order accurate upwind scheme}

A first-order accurate upwind scheme is defined by keeping the first two terms in (41)

$$
F\left(\Delta t,\left\{k_{p}\right\}\right) \approx k_{0} \frac{\partial u}{\partial x}\left(x_{i+\frac{1}{2}}, t^{n}\right)+\frac{k_{0}}{2 c} \Delta_{+} v\left(x_{i}, t^{n}\right),
$$

and approximate these using

$$
F_{i+\frac{1}{2}}\left(\Delta t, k_{0}\right) \equiv k_{0} D_{+} u_{i}^{n}+\frac{k_{0}}{2 c} \Delta_{+} v_{i}^{n} .
$$

The scheme can be efficiently evaluated using Gaussian quadrature in time with $M=1$ and quadrature node $\xi_{1}=\frac{1}{2}$, and $b_{1}=1, d_{1}=\frac{1}{2}$, to give

$$
\begin{aligned}
F_{i-\frac{1}{2}}^{1} & =F_{i-\frac{1}{2}}\left(\xi_{1} \Delta t, k_{0}=1\right), \\
g_{i} & =\Delta t c^{2} D_{+} F_{i-\frac{1}{2}}^{1}, \\
v_{i}^{n+1} & =v_{i}^{n}+g_{i}, \\
u_{i}^{n+1} & =u_{i}^{n}+\Delta t v_{i}^{n}+\frac{\Delta t}{2} g_{i} .
\end{aligned}
$$


In this case, using exact integration in time leads to the same approximation. Written out in detail, the approximation, denoted by $\mathrm{UW} 1$, is given by

$$
\begin{aligned}
& v_{i}^{n+1}=v_{i}^{n}+\Delta t c^{2} D_{+} D_{-} u_{i}^{n}+\frac{\Delta t}{2} c h_{x} D_{+} D_{-} v_{i}^{n} \\
& u_{i}^{n+1}=u_{i}^{n}+\Delta t v_{i}^{n}+\frac{\Delta t^{2}}{2} c^{2} D_{+} D_{-} u_{i}+\frac{\Delta t^{2}}{4} c h_{x} D_{+} D_{-} v_{i}^{n} .
\end{aligned}
$$

This scheme (47)-(48) differs slightly from the UW1a scheme (7)-(8) derived in Section 2. The time step restriction is larger as noted below, and this scheme is generally less dissipative (see Figure 5).

The modified equations for the scheme (47)-(48) are given by

$$
\begin{aligned}
\frac{\partial U}{\partial t} & =V+\frac{h_{x}^{2} \lambda^{2}}{12} \frac{\partial^{2} V}{\partial x^{2}}+\mathcal{O}\left(\left(\Delta t+h_{x}\right)^{3}\right) \\
\frac{\partial V}{\partial t} & =c^{2} \frac{\partial^{2} U}{\partial x^{2}}+\frac{c h_{x}}{2}(1-\lambda) \frac{\partial^{2} V}{\partial x^{2}}+\frac{c^{2} h_{x}^{2}}{12}\left(1-3 \lambda+\lambda^{2}\right) \frac{\partial^{4} U}{\partial x^{4}}+\mathcal{O}\left(\left(\Delta t+h_{x}\right)^{3}\right)
\end{aligned}
$$

where, as before, $\lambda=c \Delta t / h_{x}$. This system of equations for $U$ and $V$ can be written as decoupled equations by differentiating with respect to time and substitution. This results in the set of decoupled modified equations

$$
\begin{aligned}
& \frac{\partial^{2} U}{\partial t^{2}}=c^{2} \frac{\partial^{2} U}{\partial x^{2}}+\frac{c h_{x}}{2}(1-\lambda) \frac{\partial^{3} U}{\partial t \partial x^{2}}+\frac{c^{2} h_{x}^{2}}{12}\left(1-3 \lambda+2 \lambda^{2}\right) \frac{\partial^{4} U}{\partial x^{4}}+\mathcal{O}\left(\left(\Delta t+h_{x}\right)^{3}\right), \\
& \frac{\partial^{2} V}{\partial t^{2}}=c^{2} \frac{\partial^{2} V}{\partial x^{2}}+\frac{c h_{x}}{2}(1-\lambda) \frac{\partial^{3} V}{\partial t \partial x^{2}}+\frac{c^{2} h_{x}^{2}}{12}\left(1-3 \lambda+2 \lambda^{2}\right) \frac{\partial^{4} V}{\partial x^{4}}+\mathcal{O}\left(\left(\Delta t+h_{x}\right)^{3}\right) .
\end{aligned}
$$

Note that the modified equations for $U$ and $V$ are the same to the order given. From equations (49) and (50) we see that (for $\lambda \neq 1$ ) the approximation is first order accurate in space, $O\left(h_{x}\right)$, and first order accurate in time, $O(\Delta t)$ (note the definition of $\lambda$ ). The terms proportional to $h_{x}$ on the right hand sides of (50) and (49) represent dissipation terms which will damp the solution (higher-frequencies will be damped more strongly) provided $\lambda \in(0,1)$. The terms proportional to $h_{x}^{2}$ on the right hand sides add dispersion to the equations. The damping and dispersion properties can be seen by Fourier transforming the equations.

Remark: The modified equations (50)-(49) indicate that at $\lambda=1$ the scheme actually becomes higher than first-order accurate. In fact scheme UW1 has an infinite order of accuracy (spectrally accurate) for $\lambda=1$ (see below).

Lemma 3.3. The UW1 scheme (47)-(48) is first-order accurate and stable provided

$$
\Delta t \leq \frac{h_{x}}{c}
$$

Proof The accuracy and stability of the scheme can be determined through a normal mode analysis [15]. To this end we seek solutions to (47)-(48) of the form

$$
\left[\begin{array}{l}
u_{j}^{n} \\
v_{j}^{n}
\end{array}\right]=z^{n}\left[\begin{array}{l}
\hat{u} \\
\hat{v}
\end{array}\right] e^{2 \pi i k x_{j}},
$$

where $z$ is a complex valued amplification factor, and $\hat{u}$ and $\hat{v}$ are Fourier coefficients. The wave number $k$ takes the discrete values $k=0, \pm 1, \pm 2, \ldots \pm N / 2$ (in the case $N$ is an even integer). Substitution into (47) and (48) leads to an eigenvalue problem for $z$ with eigenvector $[\hat{u} \hat{v}]^{T}$,

$$
\left[\begin{array}{cc}
1-z-\frac{\lambda^{2}}{2} \hat{\xi}^{2} & \Delta t\left(1-\frac{\lambda}{4} \hat{\xi}^{2}\right) \\
-\frac{1}{\Delta t} \lambda^{2} \hat{\xi}^{2} & 1-z-\frac{\lambda}{2} \hat{\xi}^{2}
\end{array}\right]\left[\begin{array}{l}
\hat{u} \\
\hat{v}
\end{array}\right]=\left[\begin{array}{l}
0 \\
0
\end{array}\right]
$$

Here $\xi=2 \pi k / N$, with $\xi \in[-\pi, \pi]$, while $\hat{\xi}^{2}=4 \sin ^{2}(\xi / 2)$ is the Fourier symbol of $-\Delta_{+} \Delta_{-}$. Nontrivial solutions to this problem exist when the determinant is zero. This leads to a quadratic equation for $z$ with roots $z=z_{ \pm}$where

$$
\begin{aligned}
z_{ \pm} & =1-b \pm \sqrt{b^{2}-a}, \\
a & =\lambda^{2} \hat{\xi}^{2}, \quad b=\frac{1}{4}\left(a+\lambda \hat{\xi}^{2}\right) .
\end{aligned}
$$


Expanding (52) for small $|\xi|$ yields

$$
z_{ \pm}=1 \pm i \lambda \xi-\frac{1}{4} \lambda(1+\lambda) \xi^{2}+\mathcal{O}\left(\xi^{3}\right)=e^{ \pm i \lambda \xi}+\mathcal{O}\left(\xi^{2}\right) .
$$

Since $z=\exp ( \pm i \lambda \xi)$ corresponds to the exact solution to the continuous problem, it follows that $z_{ \pm}$are second-order accurate approximations to $\exp ( \pm i \lambda \xi)$ and thus the overall discrete solution is first-order accurate at times of order 1 (i.e. after $\mathcal{O}(1 / \Delta t)$ time-steps). Stability requires that the roots satisfy $\left|z_{ \pm}\right| \leq 1$. Contour plots of the magnitude of the two roots as a function of $\xi \in[-\pi, \pi]$ and $\lambda \in[0,1.5]$ are shown in Figure 6 . The scheme is seen to be stable for $\lambda \leq 1$. Indeed, assuming $\lambda \leq 1$ it is easily shown that

$$
\left|z_{ \pm}\right|^{2}=1-\frac{\hat{\xi}^{2}}{2} \lambda(1-\lambda)
$$

and thus $\left|z_{ \pm}\right| \leq 1$ for $\lambda \leq 1$ since $\hat{\xi}^{2} \leq 4$. The time-step restriction (51) follows. For a fully algebraic proof of this result see the proof for the two-dimensional scheme, Lemma 4.2, which includes the one-dimensional result as a special case.

Remark: For $\lambda=1$ the roots $z_{ \pm}$in (52) reduce to

$$
z_{ \pm}=\cos (\xi) \pm i \sin (\xi)=e^{ \pm i \xi}
$$

This shows that scheme UW1 has spectral accuracy when $\lambda=1$. The only error comes from representing the initial conditions as a finite discrete Fourier series.
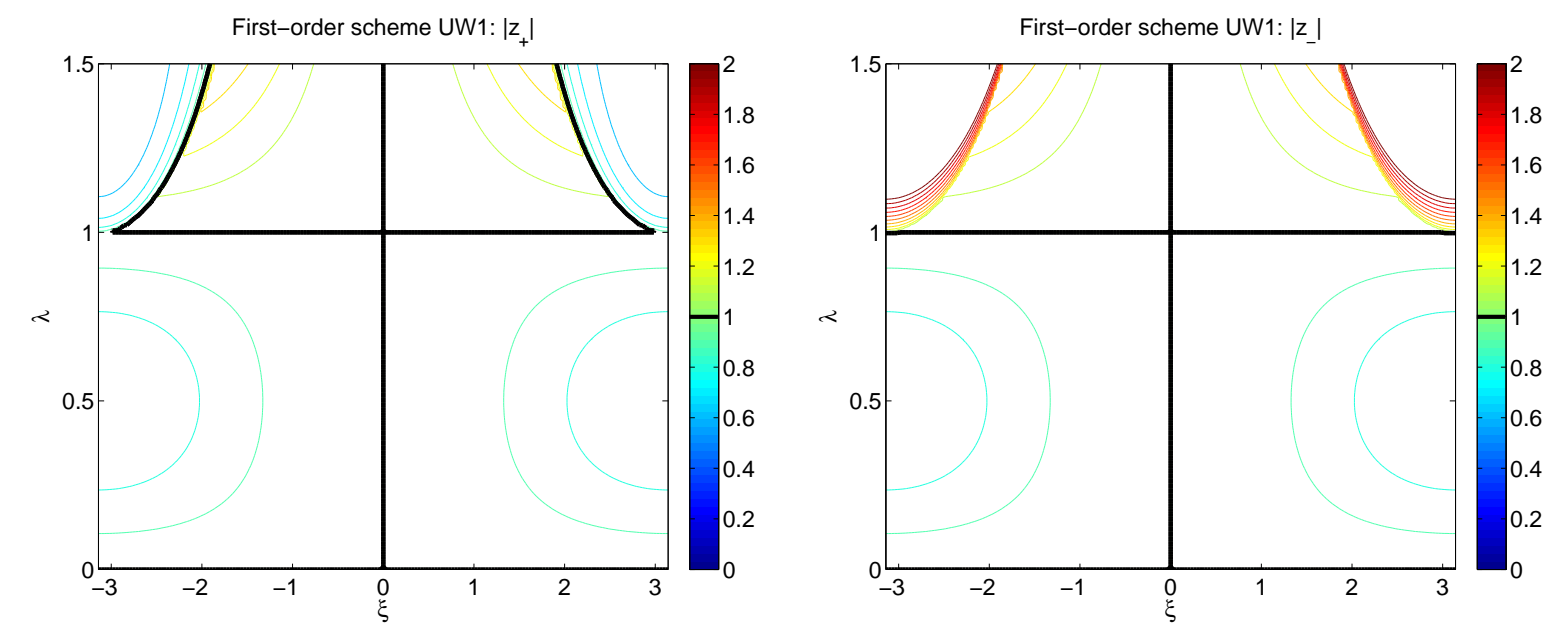

Figure 6: Region of stability for the first-order accurate scheme UW1: contours of the magnitude of the two roots $z_{ \pm}$, as a function of the CFL parameter $\lambda$ and the Fourier parameter $\xi$. The contour level 1 is shown as a thick black line. The scheme is unstable where the magnitude of either root exceeds 1 .

\subsection{A second-order accurate scheme and a high-resolution scheme}

A second-order accurate upwind scheme is defined by keeping the first five terms in (41)

$$
\begin{aligned}
F\left(\Delta t,\left\{k_{p}\right\}\right) & \approx k_{0} \frac{\partial u}{\partial x}\left(x_{i+\frac{1}{2}}, t^{n}\right)+\frac{k_{0}}{2 c} \Delta_{+} v\left(x_{i}, t^{n}\right) \\
& +k_{1} \Delta t \frac{\partial v}{\partial x}\left(x_{i+\frac{1}{2}}, t^{n}\right)+\frac{k_{1} c \Delta t}{2} \Delta_{+} \frac{\partial^{2} u}{\partial x^{2}}\left(x_{i}, t^{n}\right)-\frac{k_{0} h_{x}}{2 c} \mathcal{A}_{+} \frac{\partial v}{\partial x}\left(x_{i}, t^{n}\right),
\end{aligned}
$$

and using the approximation

$$
F_{i+\frac{1}{2}}\left(\Delta t, k_{0}, k_{1}\right) \equiv k_{0} D_{+} u_{i}^{n}+\frac{k_{0}}{2 c} \Delta_{+} v_{i}^{n}+k_{1} \Delta t D_{+} v_{i}^{n}+\frac{k_{1} c \Delta t}{2} \Delta_{+} D_{+} D_{-} u_{i}^{n}-\frac{k_{0} h_{x}}{2 c} \mathcal{A}_{+} D_{0} v_{i}^{n} .
$$

The scheme using exact time integration is then defined by

$$
\begin{aligned}
& \mathcal{F}_{i+\frac{1}{2}}^{v}\left(t^{n}, \Delta t\right) \approx F_{i+\frac{1}{2}}\left(\Delta t, k_{0}=1, k_{1}=\frac{1}{2}\right), \\
& \mathcal{F}_{i+\frac{1}{2}}^{u}\left(t^{n}, \Delta t\right) \approx F_{i+\frac{1}{2}}\left(\Delta t, k_{0}=\frac{1}{2}, k_{1}=\frac{1}{6}\right) .
\end{aligned}
$$


The scheme using Gaussian quadrature in time with $M=1$ and quadrature node $\xi_{1}=\frac{1}{2}$, and $b_{1}=1, d_{1}=\frac{1}{2}$, is defined by

$$
\begin{gathered}
F_{i+\frac{1}{2}}^{1}=F_{i+\frac{1}{2}}\left(\xi_{1} \Delta t, k_{0}=1, k_{1}=1\right), \\
\mathcal{F}_{i+\frac{1}{2}}^{v}\left(t^{n}, \Delta t\right)=F_{i+\frac{1}{2}}^{1}, \quad \mathcal{F}_{i+\frac{1}{2}}^{u}\left(t^{n}, \Delta t\right)=\frac{1}{2} F_{i+\frac{1}{2}}^{1}
\end{gathered}
$$

The Gaussian quadrature scheme can be efficiently evaluated as

$$
\begin{aligned}
g_{i} & =\Delta t c^{2} D_{+} F_{i-\frac{1}{2}}^{1}, \\
v_{i}^{n+1} & =v_{i}^{n}+g_{i}, \\
u_{i}^{n+1} & =u_{i}^{n}+\Delta t v_{i}^{n}+\frac{\Delta t}{2} g_{i} .
\end{aligned}
$$

Writing out the approximations in detail leads to the scheme (denoted by UW2)

$$
\begin{aligned}
v_{i}^{n+1}=v_{i}^{n} & +\Delta t c^{2} D_{+} D_{-} u_{i}^{n},+\frac{\Delta t^{2}}{2} c^{2} D_{+} D_{-} v_{i}^{n} \\
& +\frac{\Delta t^{2}}{4} c^{3} h_{x}\left(D_{+} D_{-}\right)^{2} u_{i}^{n}-\frac{\Delta t}{8} c h_{x}^{3}\left(D_{+} D_{-}\right)^{2} v_{i}^{n} \\
u_{i}^{n+1}=u_{i}^{n}+\Delta t v_{i}^{n} & +\frac{\Delta t^{2}}{2} c^{2} D_{+} D_{-} u_{i}+\frac{\Delta t^{3}}{4} c^{2} D_{+} D_{-} v_{i}^{n} \\
& +\frac{\Delta t^{3}}{8} c^{3} h_{x}\left(D_{+} D_{-}\right)^{2} u_{i}^{n}-\frac{\Delta t^{2}}{16} c h_{x}^{3}\left(D_{+} D_{-}\right)^{2} v_{i}^{n} .
\end{aligned}
$$

As for the case of first-order systems, slope limiters can be introduced into the approximation to reduce oscillations near discontinuities in the solution or its derivatives. The limited scheme will be referred to as a high-resolution scheme following Harten [16]. The high-resolution variant of the second-order scheme (56)(57) uses a limited approximation to the term involving $\mathcal{A}_{+} \partial v\left(x_{i}, t^{n}\right) / \partial x$ in (55)

$$
\mathcal{A}_{+} \frac{\partial v}{\partial x}\left(x_{i}, t^{n}\right) \approx \frac{1}{2}\left[S_{L}\left(D_{+} v_{i+1}^{n}, D_{-} v_{i+1}^{n}\right)+S_{L}\left(D_{+} v_{i}^{n}, D_{-} v_{i}^{n}\right)\right] .
$$

Here $S_{L}$ is a slope-limiter function that in this case attempts to limit the magnitude of the discrete approximation that is used for $\partial v / \partial x$. There are many possible choices for limiter functions. A common choice, the one used here, is the minimum modulus function defined by

$$
\operatorname{MinMod}(a, b)=\left\{\begin{array}{lll}
a & \text { if } & |a|<|b| \text { and } a b>0 \\
b & \text { if } & |a| \geq|b| \text { and } a b>0 \\
0 & \text { if } & a b \leq 0
\end{array}\right.
$$

The high-resolution scheme, denoted by HR2, uses $S_{L}(a, b)=\operatorname{MinMod}(a, b)$ in $(58)$. Note that the choice $S_{L}(a, b)=\frac{1}{2}(a+b)$ results in the original centered approximation.

Figure 7 compares the solution from the first-order (UW1), second-order (UW2) and second-order highresolution (HR2) schemes for the top-hat problem (13)-(14). The time step was chosen for each scheme to be 0.9 times the maximum allowable time-step for that scheme (i.e. a CFL number equal to 0.9). The highresolution scheme HR2 is seen to have significantly smaller undershoots and over-shoots compared to UW2. The first-order scheme UW1 gives the best solution in this case although this is somewhat fortuitous since the UW1 scheme becomes spectrally accurate at $\lambda=1$. Later in Section 6.2 the discrete $L_{1}$-norm errors for this problem are shown (Figure 18). While scheme UW1 convergences more slowly than the other schemes, the $L_{1}$-norm errors are smaller than the UW2 and HR2 schemes for a wide range of $h_{x}$. These advantages of the UW1 scheme disappear, however, in two or three space dimensions since the stable time-step will lead to much smaller values of $\lambda$ being used.

Remark: It might seem that the high-resolution scheme should remain second-order accurate for smooth solutions. However, as shown in Section 6.1, while the max-norm errors in $u$ converge at order 2 , the maxnorm errors for $v$ are degraded to a convergence rate of $4 / 3$. This degradation can be traced to the lack of smoothness in the MinMod limiter. 


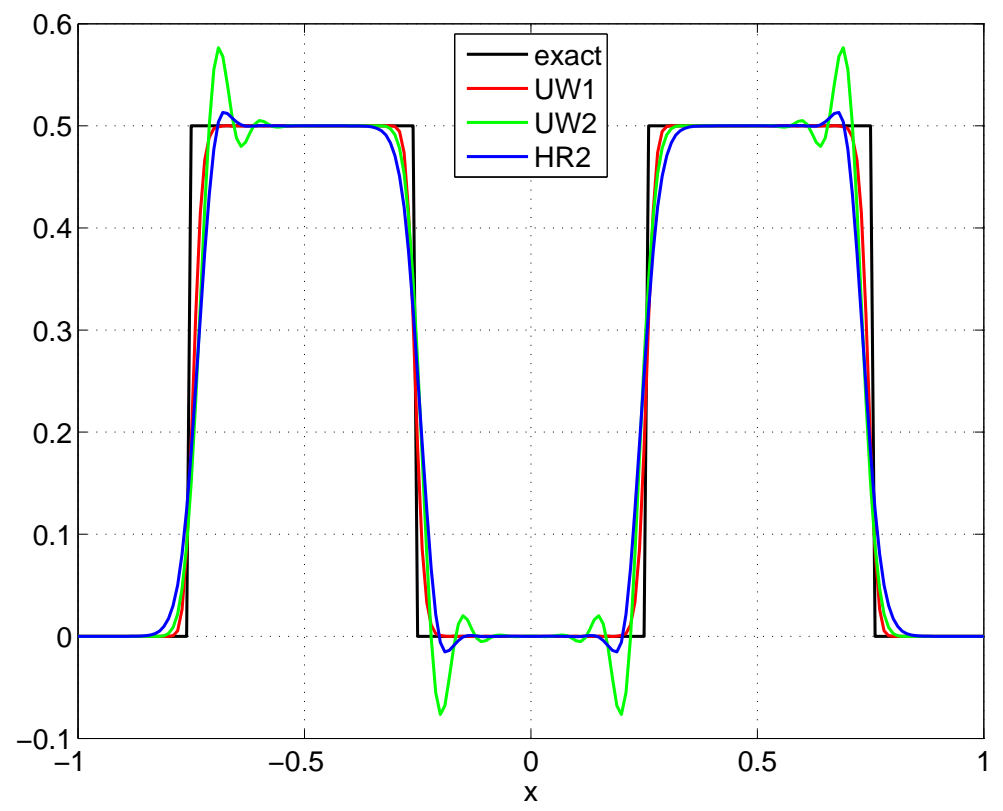

Figure 7: Results for the top hat initial condition of (13)-(14) using the first-order accurate scheme UW1, the secondorder accurate scheme UW2, and second-order accurate high-resolution scheme, HR2. Solution $u$ at $t=0.5$ using $N=201$ grid points.

In order to understand the accuracy of the scheme (56)-(57), the leading terms of the modified equation are derived through Taylor expansion. Following the steps discussed previously for the first-order accurate scheme leads to the following system of modified equations

$$
\begin{aligned}
\frac{\partial^{2} U}{\partial t^{2}} & =c^{2} \frac{\partial^{2} U}{\partial x^{2}}+c^{2} h_{x}^{2} M_{2}(\lambda) \frac{\partial^{4} U}{\partial x^{4}}+c h_{x}^{3} C_{2}(\lambda) \frac{\partial^{5} U}{\partial t \partial x^{4}}+\mathcal{O}\left(\left(\Delta t+h_{x}\right)^{4}\right), \\
\frac{\partial^{2} V}{\partial t^{2}} & =c^{2} \frac{\partial^{2} V}{\partial x^{2}}+c^{2} h_{x}^{2} M_{2}(\lambda) \frac{\partial^{4} V}{\partial x^{4}}+c h_{x}^{3} C_{2}(\lambda) \frac{\partial^{5} V}{\partial t \partial x^{4}}+\mathcal{O}\left(\left(\Delta t+h_{x}\right)^{4}\right), \\
M_{2}(\lambda) & =\frac{1}{12}\left(1+3 \lambda-\lambda^{2}\right), \quad C_{2}(\lambda)=-\frac{1}{8}\left(1+\lambda^{2}\right) .
\end{aligned}
$$

The modified equations (60)- (61) are second order accurate approximations to the true equations in both space and time (i.e. $O\left(\Delta x^{2}\right)$ for $\Delta t=\lambda \Delta x / c$ ). The first error term in the modified equations is a dispersion term and the second is a dissipation term (provided $C_{2}(\lambda) \leq 0$ ).

Lemma 3.4. The scheme UW2 (56)-(57) is second-order accurate and stable provided

$$
\Delta t \leq \Lambda_{2} \frac{h_{x}}{c}
$$

where $\Lambda_{2}=\frac{\sqrt{5}-1}{2} \approx 0.618034$.

Proof The accuracy and stability for the second-order accurate scheme follows the same normal mode approach as for the first-order scheme. Similar steps lead to the eigenvalue problem

$$
\left[\begin{array}{cc}
1-z-\frac{1}{2} \gamma+\frac{1}{8} \eta & \Delta t\left(1-\frac{1}{4} \gamma-\frac{1}{16} \zeta\right) \\
-\frac{1}{\Delta t}\left(\gamma-\frac{1}{4} \eta\right) & 1-z-\frac{1}{2} \gamma-\frac{1}{8} \zeta
\end{array}\right]\left[\begin{array}{l}
\hat{u} \\
\hat{v}
\end{array}\right]=\left[\begin{array}{l}
0 \\
0
\end{array}\right]
$$

where

$$
\gamma \equiv \lambda^{2} \hat{\xi}^{2}, \quad \delta \equiv \lambda \hat{\xi}^{2}, \quad \eta \equiv \delta \gamma, \quad \zeta \equiv \lambda \hat{\xi}^{4} .
$$

The two eigenvalues $z=z_{ \pm}$are given by

$$
\begin{gathered}
z_{ \pm}=1-b \pm \sqrt{b^{2}-a} \\
a=\gamma\left(1-\frac{1}{4} \delta\right), \quad b=\frac{1}{2}\left(\gamma-\frac{1}{8} \eta+\frac{1}{8} \zeta\right) .
\end{gathered}
$$


Expanding (64) for small $|\xi|$ gives

$$
z_{ \pm}=1 \pm i \lambda \xi-\frac{1}{2}(\lambda \xi)^{2} \pm(i)^{3} \frac{\lambda}{24}\left(1+3 \lambda-\lambda^{2}\right) \xi^{3}+\mathcal{O}\left(\xi^{4}\right)=e^{ \pm i \lambda \xi}+\mathcal{O}\left(\xi^{3}\right)
$$

and thus the solution is second-order accurate. Note that the coefficient of the $\xi^{3}$ term in (66) is closely related to the coefficient $M_{2}(\lambda)$ of the leading error term in the modified equation (60). This is not a coincidence and the general relationship is discussed later in Section 5 (see Figure 13). Contour plots of the magnitude of the two roots are shown in Figure 8. The figures show that the scheme first becomes unstable on $\xi= \pm \pi$. These are the most oscillatory plus-minus modes. For $\xi= \pm \pi$ the stability condition reduces to

$$
\lambda^{2}+\lambda \leq 1,
$$

which leads to the time-step restriction (63). For a fully algebraic proof of this result see the proof for the two-dimensional scheme, Lemma 4.3, which includes the one-dimensional result as a special case.
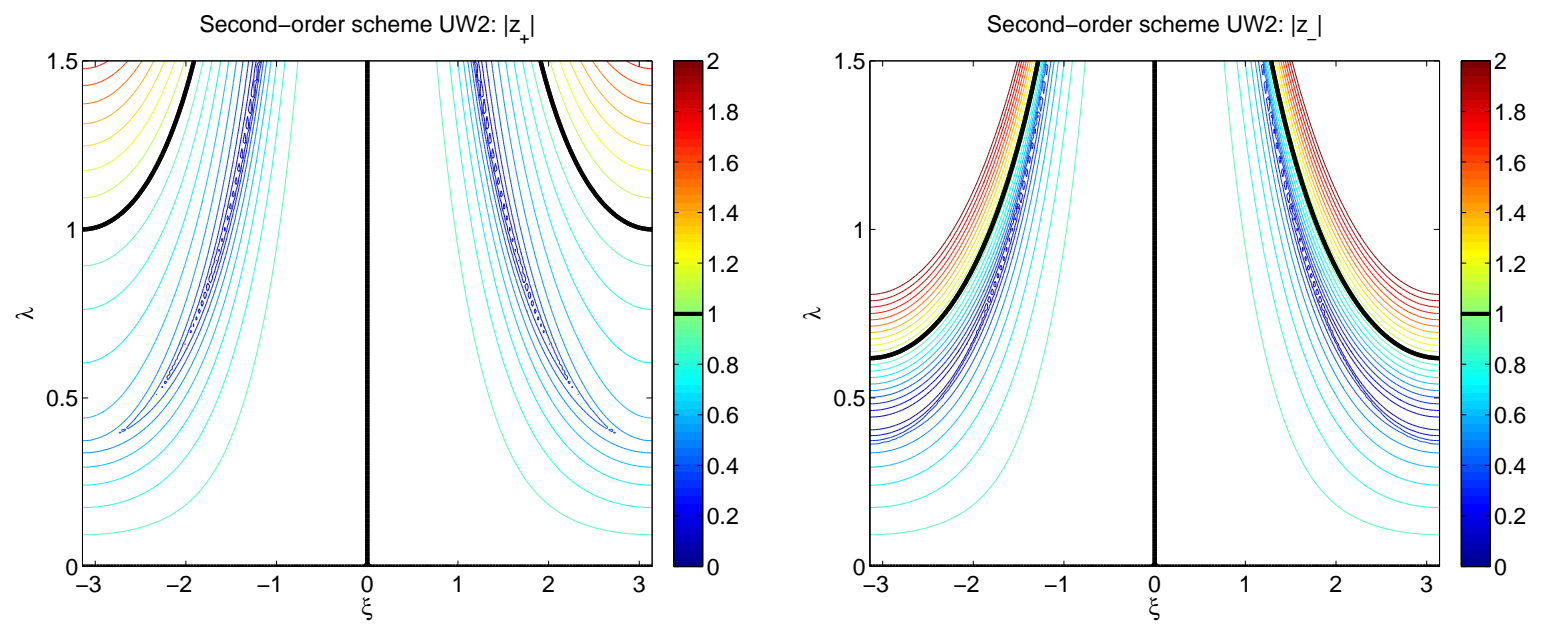

Figure 8: Region of stability for the second-order accurate scheme UW2: contours of the magnitude of the two roots $z_{ \pm}$as a function of the CFL parameter $\lambda$ and the Fourier parameter $\xi$. The contour level 1 is shown as a thick black line. The scheme is stable for $\lambda \leq \frac{\sqrt{5}-1}{2} \approx 0.618$. The modes which determine the stability bound are at $\xi= \pm \pi$.

\subsection{A fourth-order accurate upwind scheme}

For fourth-order accuracy, (41) is approximated using

$$
\begin{aligned}
F & \left(\Delta t,\left\{k_{p}\right\}\right) \approx k_{0} \frac{\partial u}{\partial x}\left(x_{i+\frac{1}{2}}, t^{n}\right)+\frac{k_{0}}{2 c} \Delta_{+} v\left(x_{i}, t^{n}\right) \\
& +k_{1} \Delta t \frac{\partial v}{\partial x}\left(x_{i+\frac{1}{2}}, t^{n}\right)+\frac{k_{1} c \Delta t}{2} \Delta_{+} \frac{\partial^{2} u}{\partial x^{2}}\left(x_{i}, t^{n}\right)-\frac{k_{0} h_{x}}{2 c} \mathcal{A}_{+} \frac{\partial v}{\partial x}\left(x_{i}, t^{n}\right) \\
& +\left(\frac{k_{2} c^{2} \Delta t^{2}}{2}-\frac{k_{0} h_{x}^{2}}{24}\right) \frac{\partial^{3} u}{\partial x^{3}}\left(x_{i+\frac{1}{2}}, t^{n}\right)+\left(\frac{k_{2} c \Delta t^{2}}{4}+\frac{k_{0} h_{x}^{2}}{24 c}\right) \Delta_{+} \frac{\partial^{2} v}{\partial x^{2}}\left(x_{i}, t^{n}\right)-\frac{k_{1} c \Delta t h_{x}}{2} \mathcal{A}_{+} \frac{\partial^{3} u}{\partial x^{3}}\left(x_{i}, t^{n}\right) \\
& +\left(\frac{k_{3} c^{2} \Delta t^{3}}{6}-\frac{k_{1} \Delta t h_{x}^{2}}{24}\right) \frac{\partial^{3} v}{\partial x^{3}}\left(x_{i+\frac{1}{2}}, t^{n}\right)+\left(\frac{k_{3} c^{2} \Delta t^{3}}{12}+\frac{k_{1} c \Delta t h_{x}^{2}}{24}\right) \Delta_{+} \frac{\partial^{4} u}{\partial x^{4}}\left(x_{i}, t^{n}\right) \\
& -\frac{k_{2} c \Delta t^{2} h_{x}}{4} \mathcal{A}_{+} \frac{\partial^{3} v}{\partial x^{3}}\left(x_{i}, t^{n}\right),
\end{aligned}
$$


with the discrete approximation

$$
\begin{aligned}
F_{i+\frac{1}{2}}\left(\Delta t, k_{0}, k_{1}, k_{2}, k_{3}\right) \equiv & k_{0} D_{+}\left(1-\frac{h_{x}^{2}}{24} D_{+} D_{-}\right) u_{i}^{n}+\frac{k_{0}}{2 c} \Delta_{+} v_{i}^{n} \\
& +k_{1} \Delta t D_{+}\left(1-\frac{h_{x}^{2}}{24} D_{+} D_{-}\right) v_{i}^{n}+\frac{k_{1} c \Delta t}{2} \Delta_{+} D_{4 h}^{(2)} u_{i}^{n} \\
& -\frac{k_{0} h_{x}}{2 c} \mathcal{A}_{+} D_{0}\left(1-\frac{h_{x}^{2}}{6} D_{+} D_{-}\right) v_{i}^{n}+\left(\frac{k_{2} c^{2} \Delta t^{2}}{2}-\frac{k_{0} h_{x}^{2}}{24}\right) D_{+}^{2} D_{-} u_{i}^{n} \\
& +\left(\frac{k_{2} c \Delta t^{2}}{4}+\frac{k_{0} h_{x}^{2}}{24 c}\right) \Delta_{+} D_{4 h}^{(2)} v_{i}^{n}-\frac{k_{1} c \Delta t h_{x}}{2} \mathcal{A}_{+} D_{0} D_{+} D_{-} u_{i}^{n} \\
& +\left(\frac{k_{3} c^{2} \Delta t^{3}}{6}-\frac{k_{1} \Delta t h_{x}^{2}}{24}\right) D_{+}^{2} D_{-} v_{i}^{n}+\left(\frac{k_{3} c^{3} \Delta t^{3}}{12}+\frac{k_{1} c \Delta t h_{x}^{2}}{24}\right) \Delta_{+}\left(D_{+} D_{-}\right)^{2} u_{i}^{n} \\
& -\frac{k_{2} c \Delta t^{2} h_{x}}{4} \mathcal{A}_{+} D_{0} D_{+} D_{-} v_{i}^{n} .
\end{aligned}
$$

Here

$$
D_{4 h}^{(2)}=D_{+} D_{-}\left(1-\frac{h_{x}^{2}}{12} D_{+} D_{-}\right)
$$

defines a cell-centered fourth-order accurate approximation to $\partial^{2} / \partial x^{2}$. Gaussian quadrature in time with $M=2$ uses the quadrature nodes $\xi_{1}=\frac{1}{2}(1-\sqrt{3})$ and $\xi_{2}=\frac{1}{2}(1+\sqrt{3})$ along with $b_{1}=b_{2}=\frac{1}{2}, d_{1}=\frac{1}{4}+\frac{\sqrt{3}}{12}$, $d_{2}=\frac{1}{4}-\frac{\sqrt{3}}{12}$. The Gaussian quadrature scheme can be evaluated using

$$
\begin{aligned}
& F_{i+\frac{1}{2}}^{1}=F_{i+\frac{1}{2}}\left(\xi_{1} \Delta t,\{1\}\right), \quad F_{i+\frac{1}{2}}^{2}=F_{i+\frac{1}{2}}\left(\xi_{2} \Delta t,\{1\}\right), \\
& F_{i+\frac{1}{2}}^{v}=b_{1} F_{i+\frac{1}{2}}^{1}+b_{2} F_{i+\frac{1}{2}}^{2}, \quad F_{i+\frac{1}{2}}^{u}=d_{1} F_{i+\frac{1}{2}}^{1}+d_{2} F_{i+\frac{1}{2}}^{2}, \\
& v_{i}^{n+1}=v_{i}^{n}+c^{2} \Delta t D_{+} F_{i+\frac{1}{2}}^{v} \\
& u_{i}^{n+1}=u_{i}^{n}+\Delta t v_{i}^{n}+c^{2} \Delta t^{2} D_{+} F_{i+\frac{1}{2}}^{u} .
\end{aligned}
$$

Written out in detail, the fourth-order accurate upwind scheme is (denoted by UW4)

$$
\begin{aligned}
v_{i}^{n+1} & =v_{i}^{n}+c^{2} \Delta t G_{i}\left(k_{0}=1, k_{1}=\frac{1}{2}, k_{2}=\frac{1}{3}, k_{4}=\frac{1}{4}\right), \\
u_{i}^{n+1} & =u_{i}^{n}+\Delta t v_{i}^{n}+c^{2} \Delta t^{2} G_{i}\left(k_{0}=\frac{1}{2}, k_{1}=\frac{1}{6}, k_{2}=\frac{1}{12}, k_{3}=\frac{1}{18}\right), \\
G_{i}\left(k_{p}\right) & =k_{0} D_{4 h}^{(2)} u_{i}^{n}+k_{1} \Delta t D_{4 h}^{(2)} v_{i}^{n}+\frac{k_{2}}{2} c^{2} \Delta t^{2}\left(D_{+} D_{-}\right)^{2} u_{i}+\frac{k_{3}}{6} c^{2} \Delta t^{3}\left(D_{+} D_{-}\right)^{2} v_{i} \\
& +\left(\frac{5 k_{0}}{288 c} h_{x}^{5}-\frac{k_{2}}{12} c \Delta t^{2} h_{x}^{3}\right)\left(D_{+} D_{-}\right)^{3} v_{i}+\left(-\frac{k_{1}}{8} c \Delta t h_{x}^{3}+\frac{k_{3}}{12} c^{3} \Delta t^{3} h_{x}\right)\left(D_{+} D_{-}\right)^{3} u_{i} .
\end{aligned}
$$

The stencil coefficients for this scheme are given in Appendix A.1.

Lemma 3.5. The scheme UW4 (73)-(75) is fourth-order accurate and stable provided

$$
\Delta t \leq \Lambda_{4} \frac{h_{x}}{c},
$$

where $\Lambda_{4}$ is given by the smallest real positive root of the polynomial equation

$$
4 \lambda^{5}-14 \lambda^{4}-12 \lambda^{3}+12 \lambda^{2}+15=0
$$

and is given approximately by $\Lambda_{4} \approx 1.09$.

Proof To determine the accuracy and stability of this fourth order scheme, a normal mode analysis is performed as before, giving the eigenvalue problem

$$
\left[\begin{array}{cc}
1-z-\frac{\lambda^{2}}{2} \beta_{4}+\frac{\lambda^{4}}{24} \hat{\xi}^{4}+\frac{\lambda^{3}}{1}\left(\frac{1}{48}-\frac{1}{216} \lambda^{2}\right) \hat{\xi}^{6} & \Delta t\left(1-\frac{\lambda^{2}}{6} \beta_{4}+\frac{\lambda^{4}}{108} \hat{\xi}^{4}+\frac{\lambda}{36}\left(-\frac{5}{16}+\frac{1}{4} \lambda^{2}\right) \hat{\xi}^{6}\right) \\
\left.\frac{1}{\Delta t}\left(-\lambda^{2} \beta_{4}+\frac{\lambda^{4}}{6} \hat{\xi}^{4}\right)+\frac{\lambda^{3}}{1}\left(\frac{1}{16}-\frac{1}{48} \lambda^{2}\right) \hat{\xi}^{6}\right) & 1-z-\frac{\lambda^{2}}{2} \beta_{4}+\frac{\lambda^{4}}{24} \hat{\xi}^{4}+\frac{\lambda}{36}\left(-\frac{5}{8}+\lambda^{2}\right) \hat{\xi}^{6}
\end{array}\right]\left[\begin{array}{l}
\hat{u} \\
\hat{v}
\end{array}\right]=\left[\begin{array}{l}
0 \\
0
\end{array}\right],
$$


where $\beta_{4}=\hat{\xi}^{2}\left(1+\frac{1}{12} \hat{\xi}^{2}\right)$.

The determinant condition is of the same form as before and there will be two roots $z_{ \pm}$. Expanding the roots for small $|\xi|$ we find that

$$
\begin{aligned}
z_{ \pm} & =1 \pm i \lambda \xi+\frac{1}{2}(i \lambda \xi)^{2} \pm \frac{1}{3 !}(i \lambda \xi)^{3}+\frac{1}{4 !}(i \lambda \xi)^{4} \pm i \frac{\lambda}{2} M_{4}(\lambda) \xi^{5}+\mathcal{O}\left(\xi^{6}\right) \\
& =e^{ \pm i \lambda \xi}+\mathcal{O}\left(\xi^{5}\right)
\end{aligned}
$$

showing that the solution is fourth-order accurate (the coefficient $M_{4}(\lambda)$ is given below (77)). Contour plots of the magnitude of the two roots are shown in Figure 9. Interestingly, the modes which determine the stability bound occur near the lowest frequency mode, $\xi=0$ (The mode $\xi=0$ is itself stable with amplitude 1). For $|\xi| \ll 1$

$$
\left|z_{ \pm}\right|^{2}=1-P_{6}(\lambda) \xi^{6}+\mathcal{O}\left(\xi^{8}\right)
$$

where $P_{6}(\lambda)$ is a polynomial in $\lambda$. The requirement $\left|z_{ \pm}\right|^{2} \leq 1$ requires $P_{6}(\lambda) \geq 0$. This leads to the stability limit being given by the smallest real positive root of the polynomial equation (76), resulting in a stability bound of $\Lambda_{4} \approx 1.09$.

The modified equation for the fourth order scheme is

$$
\begin{aligned}
\frac{\partial^{2} U}{\partial t^{2}} & =c^{2} \frac{\partial^{2} U}{\partial x^{2}}+c^{2} h_{x}^{4} M_{4}(\lambda) \frac{\partial^{6} U}{\partial x^{6}}+c h_{x}^{5} C_{4}(\lambda) \frac{\partial^{7} U}{\partial t \partial x^{6}}+\mathcal{O}\left(\left(\Delta t+h_{x}\right)^{6}\right), \\
M_{4}(\lambda) & =\frac{1}{2600}\left(-24-135 \lambda+60 \lambda^{2}+45 \lambda^{3}-16 \lambda^{4}\right), \\
C_{4}(\lambda) & =\frac{1}{864}\left(15+12 \lambda^{2}-12 \lambda^{3}-14 \lambda^{4}+4 \lambda^{5}\right),
\end{aligned}
$$

where $V$ satisfies the same equation as $U$. As was the case for the modified equations (60)-(61) for the second-order accurate scheme UW2, the leading error term in the modified equations for scheme UW4 is a dispersive term while the following term provides the dissipation (assuming $C_{4}(\lambda) \geq 0$ ).
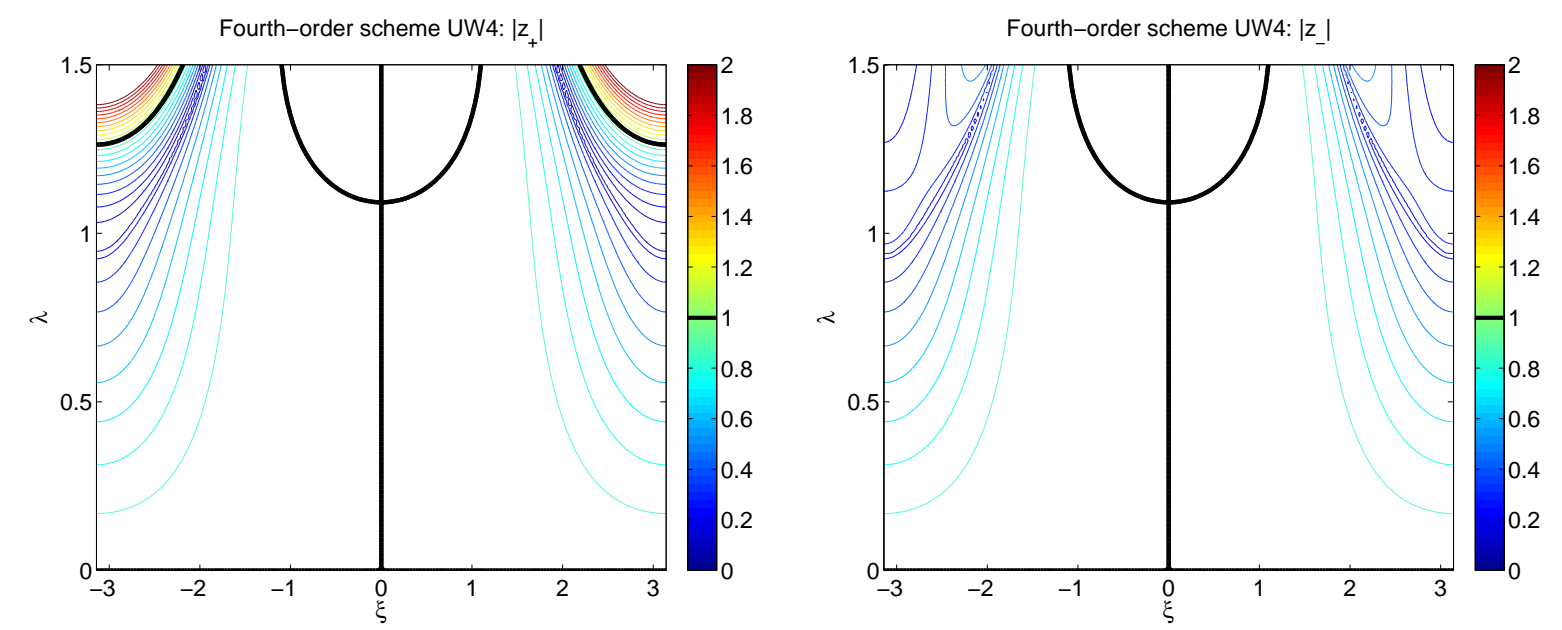

Figure 9: Region of stability for the fourth-order accurate scheme UW4: contours of the magnitude of the two roots $z_{ \pm}$as functions of the CFL parameter $\lambda$ and the Fourier parameter $\xi$. The contour level 1 is shown as a thick black line. The modes which determine the stability bound are near $\xi=0$ for $\lambda \approx 1.09$.

\subsection{A sixth-order accurate upwind scheme}

The sixth-order accurate upwind scheme UW6 is constructed following the same procedures as outlined for the lower order schemes. We do not write down all the details here since the process for developing the scheme should be clear from the previous cases. The stencil coefficients for the UW6 scheme are given in Appendix A.2. 
Lemma 3.6. The scheme UW6 is sixth-order accurate and stable provided

$$
\Delta t \leq \Lambda_{6} \frac{h_{x}}{c},
$$

where $\Lambda_{6}$ is given by the smallest real positive root of the polynomial equation

$$
\begin{array}{r}
-303750+139500 \lambda+459000 \lambda^{2}-99000 \lambda^{3}-274600 \lambda^{4}+55200 \lambda^{5}+61600 \lambda^{6} \\
-33615 \lambda^{7}-4836 \lambda^{8}+5262 \lambda^{9}+1456 \lambda^{10}+165 \lambda^{11}-208 \lambda^{12}=0,
\end{array}
$$

and is given approximately by $\Lambda_{6} \approx 0.9502$.

Proof The accuracy and stability analysis of the UW6 scheme proceeds as before. The roots $z_{ \pm}$satisfy

$$
z_{ \pm}=e^{ \pm i \lambda \xi} \mp i \frac{\lambda}{2} M_{6}(\lambda) \xi^{7}+\mathcal{O}\left(\xi^{8}\right)
$$

and thus the solution is sixth-order accurate (the coefficient $M_{6}(\lambda)$ is given below (80)). Figure 10 shows contours of the magnitudes of the two roots $z_{ \pm}$from which it is seen that the high-frequency modes determine the stability bound. Examination of the magnitude of the roots $z_{ \pm}$for $\xi= \pm \pi$ leads to the the stability condition (78).

The modified equation for scheme UW6 is given by ( $V$ satisfies the same equation as $U$ )

$$
\begin{aligned}
\frac{\partial^{2} U}{\partial t^{2}} & =c^{2} \frac{\partial^{2} U}{\partial x^{2}}-c^{2} h_{x}^{6} M_{6}(\lambda) \frac{\partial^{8} U}{\partial x^{8}}+c h_{x}^{7} C_{6}(\lambda) \frac{\partial^{9} U}{\partial t \partial x^{8}}+\mathcal{O}\left(\left(\Delta t+h_{x}\right)^{8}\right), \\
M_{6}(\lambda) & =\frac{1}{1008000}\left(1800+14700 \lambda-4900 \lambda^{2}-7000 \lambda^{3}+2100 \lambda^{4}-197 \lambda^{5}\right), \\
C_{6}(\lambda) & =\frac{1}{864000}\left(-3100-4100 \lambda^{2}+2100 \lambda^{3}+3300 \lambda^{4}-1200 \lambda^{5}-513 \lambda^{6}+126 \lambda^{7}\right) .
\end{aligned}
$$

As for schemes UW2 and UW4, the error terms in the modified equations for the sixth-order accurate scheme begin with a dispersive term, followed by a dissipation term. A summary of the form of the modified equations for the different schemes is given in the Section 5 (see Figure 13).
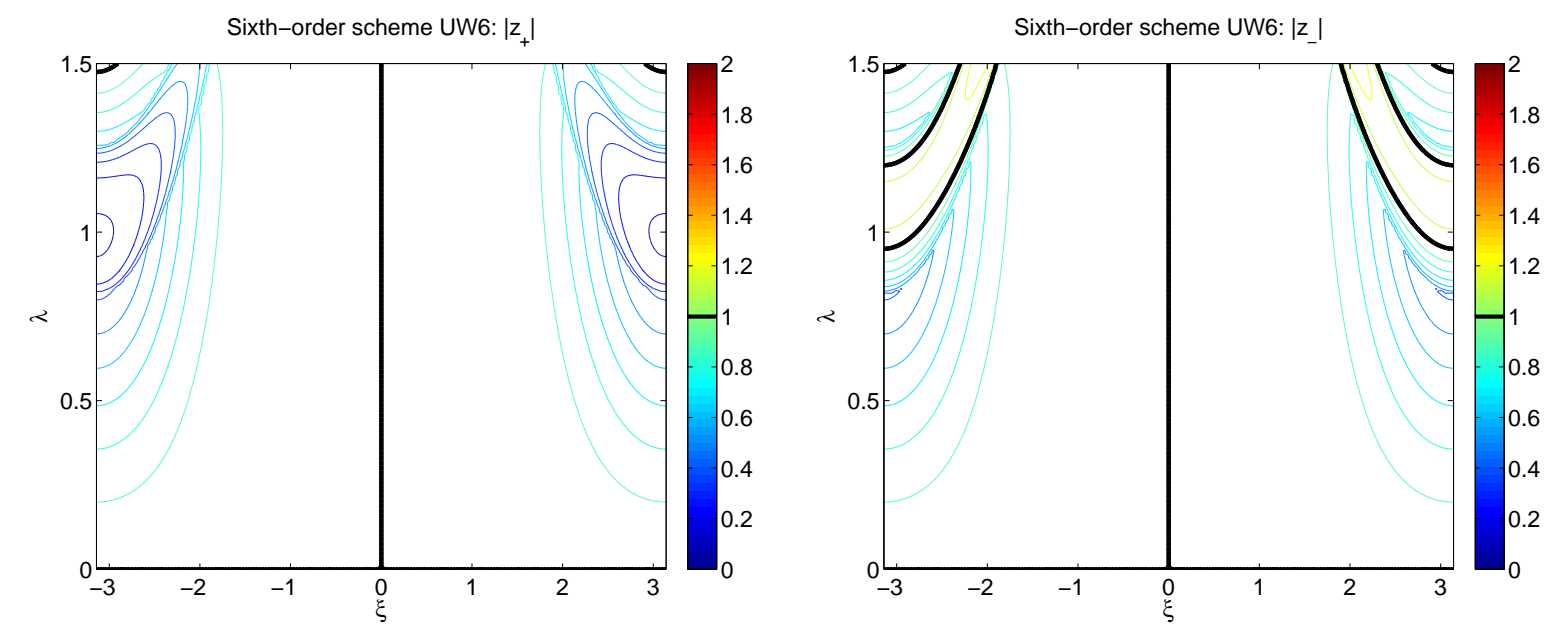

Figure 10: Region of stability for the sixth-order accurate scheme UW6: contours of the magnitude of the two roots $z_{ \pm}$as functions of the CFL parameter $\lambda$ and the Fourier parameter $\xi$. The contour level 1 is shown as a thick black line. The scheme first becomes unstable on $\xi= \pm \pi$ for $\lambda \approx .95$.

\section{Upwind schemes in two space dimensions}

The one-dimensional upwind schemes devised in previous sections can be extended to more space dimensions and in this section we show how to construct schemes in two space dimensions. The generalization to three space dimensions should be clear given the subsequent discussion. The approach in two-dimensions 
will closely follow the developments of Section 3. The two-dimensional wave equation in second-order form will be integrated in time and put into the form of a conservation equation for the displacement, $u$, and velocity, $v$. Upwind flux functions in the $x$ - and $y$-directions will be defined to be of the same form as the one-dimensional upwind flux function (31). Space-time schemes will then be defined using the CauchyKowalewski procedure. Two-dimensional schemes with orders of accuracy up to four will be constructed and analyzed.

Consider the initial-value problem for the following second order wave equation in two space dimensions

$$
\begin{aligned}
\frac{\partial^{2} u}{\partial t^{2}} & =L u, \quad \mathbf{x} \in \Omega, \\
L & \equiv c_{x}^{2} \frac{\partial^{2}}{\partial x^{2}}+c_{y}^{2} \frac{\partial^{2}}{\partial y^{2}}, \\
u(\mathbf{x}, 0) & =u_{0}(\mathbf{x}), \quad u_{t}(\mathbf{x}, 0)=v_{0}(\mathbf{x}),
\end{aligned}
$$

where $\mathbf{x}=(x, y)$, and $u=u(x, y, t)=u(\mathbf{x}, t)$ is the displacement. Let $\Omega=[0,1]^{2}$ be the unit square and take $u$ to be periodic in both $x$ and $y$ directions. Furthermore, assume that $c_{x}$ and $c_{y}$ are constant, with $c_{x}>0$ and $c_{y}>0$. As for the one-dimensional case, we introduce the velocity $v(\mathbf{x}, t)=u_{t}(\mathbf{x}, t)$ and rewrite (81) as the equivalent system

$$
\left[\begin{array}{l}
u \\
v
\end{array}\right]_{t}=\left[\begin{array}{c}
0 \\
c_{x}^{2} u_{x}
\end{array}\right]_{x}+\left[\begin{array}{c}
0 \\
c_{y}^{2} u_{y}
\end{array}\right]_{y}+\left[\begin{array}{l}
v \\
0
\end{array}\right]
$$

\subsection{Discretization in two space dimensions}

Discrete approximations to (81) will be defined based on conservative finite differences. Introduce a uniform Cartesian grid on $\Omega$ with $\left(N_{x}+1\right) \times\left(N_{y}+1\right)$ grid points. Let $h_{x}=1 / N_{x}$ and $h_{y}=1 / N_{y}$ denote the grid spacings. Denote the grid cell centers by $\mathbf{x}_{i, j}=\left(x_{i}, y_{j}\right)=\left(\left(i+\frac{1}{2}\right) h_{x},\left(j+\frac{1}{2}\right) h_{y}\right), i=0,1, \ldots, N_{x}-1$, $j=0,1, \ldots, N_{y}-1$ and the grid vertices by $\mathbf{x}_{i-\frac{1}{2}, j-\frac{1}{2}}=\left(i h_{x}, j h_{y}\right), i=0,1, \ldots, N_{x}, j=0,1, \ldots, N_{y}$. For compactness we also write $u_{\mathbf{i}}=u_{i, j}$ and $\mathbf{x}_{\mathbf{i}}=\mathbf{x}_{i, j}$ where $\mathbf{i}=(i, j)$ is a multi-index.

Let $u_{\mathbf{i}}^{n} \approx u\left(\mathbf{x}_{\mathbf{i}}, t^{n}\right)$ and $v_{\mathbf{i}}^{n} \approx v\left(\mathbf{x}_{\mathbf{i}}, t^{n}\right)$ denote discrete approximations to the displacement and velocity. Let $D_{+x}, D_{-x}, D_{+y}, D_{-y}$, denote the forward, and backward divided difference operators in the x and y directions, and $\Delta_{+x}, \Delta_{-x}, \Delta_{+y}$, and $\Delta_{-y}$ the related undivided difference operators. Let $\mathcal{A}_{+x}$ and $\mathcal{A}_{+y}$ denote the forward averaging operators in the $x$ - and $y$-directions. For example, $D_{+x} w_{\mathbf{i}}=\left(w_{i+1, j}-w_{i, j}\right) / h_{x}$, $D_{-y} w_{\mathbf{i}}=\left(w_{i, j}-w_{i, j-1}\right) / h_{y}, \Delta_{+y} w_{\mathbf{i}}=\left(w_{i, j+1}-w_{i, j}\right)$ and $\mathcal{A}_{+y} w_{\mathbf{i}}=\left(w_{i, j+1}+w_{i, j}\right) / 2$.

Following the procedure developed in Section 3, equation (83) is integrated in time over a time step $\Delta t$ to obtain equations for $v\left(\mathbf{x}, t^{n+1}\right)$ and $u\left(\mathbf{x}, t^{n+1}\right)$ in terms of the solution at time $t^{n}$. The continuous spatial derivatives are transformed into the form of a discrete conservation law. This leads to the following formally exact differential-difference equations for the solution,

$$
\begin{aligned}
& v\left(\mathbf{x}, t^{n+1}\right)=v\left(\mathbf{x}, t^{n}\right)+c_{x}^{2} \Delta t D_{+x} \mathcal{F}_{x}^{v}\left(x-\frac{h_{x}}{2}, y, t^{n}\right)+c_{y}^{2} \Delta t D_{+y} \mathcal{F}_{y}^{v}\left(x, y-\frac{h_{y}}{2}, t^{n}\right), \\
& u\left(\mathbf{x}, t^{n+1}\right)=u\left(\mathbf{x}, t^{n}\right)+\Delta t v\left(\mathbf{x}, t^{n}\right)+c_{x}^{2} \Delta t^{2} D_{+x} \mathcal{F}_{x}^{u}\left(x-\frac{h_{x}}{2}, y, t^{n}\right)+c_{y}^{2} \Delta t^{2} D_{+y} \mathcal{F}_{y}^{u}\left(x, y-\frac{h_{y}}{2}, t^{n}\right),
\end{aligned}
$$

where

$$
\begin{aligned}
& \mathcal{F}_{x}^{v}\left(\mathbf{x}, t^{n}\right)=\frac{1}{\Delta t} \int_{0}^{\Delta t} \check{f}_{x}\left(\mathbf{x}, t^{n}+\tau\right) d \tau, \quad \mathcal{F}_{y}^{v}\left(\mathbf{x}, t^{n}\right)=\frac{1}{\Delta t} \int_{0}^{\Delta t} \check{f}_{y}\left(\mathbf{x}, t^{n}+\tau\right) d \tau \\
& \mathcal{F}_{x}^{u}\left(\mathbf{x}, t^{n}\right)=\frac{1}{\Delta t^{2}} \int_{0}^{\Delta t} \int_{0}^{\tau} \check{f}_{x}\left(\mathbf{x}, t^{n}+\tau^{\prime}\right) d \tau^{\prime} d \tau, \quad \mathcal{F}_{y}^{u}\left(\mathbf{x}, t^{n}\right)=\frac{1}{\Delta t^{2}} \int_{0}^{\Delta t} \int_{0}^{\tau} \check{f}_{y}\left(x, t^{n}+\tau^{\prime}\right) d \tau^{\prime} d \tau,
\end{aligned}
$$

and where the upwind flux functions are given by

$$
\begin{aligned}
\check{f}_{x}\left(x+\frac{h_{x}}{2}, y, t^{n}+\tau\right) \equiv & \mathcal{D}_{x} \frac{\partial u}{\partial x}\left(x+\frac{h_{x}}{2}, y, t^{n}+\tau\right) \\
& +\frac{1}{2 c_{x}}\left[\mathcal{D}_{x} v^{+x}\left(x+\frac{h_{x}}{2}, y, t^{n}+\tau\right)-\mathcal{D}_{x} v^{-x}\left(x+\frac{h_{x}}{2}, y, t^{n}+\tau\right)\right], \\
\check{f}_{y}\left(x, y+\frac{h_{y}}{2}, t^{n}+\tau\right) \equiv & \mathcal{D}_{y} \frac{\partial u}{\partial y}\left(x, y+\frac{h_{y}}{2}, t^{n}+\tau\right) \\
& +\frac{1}{2 c_{y}}\left[\mathcal{D}_{y} v^{+y}\left(x, y+\frac{h_{y}}{2}, t^{n}+\tau\right)-\mathcal{D}_{y} v^{-y}\left(x, y+\frac{h_{y}}{2}, t^{n}+\tau\right)\right] .
\end{aligned}
$$


Here the differential-difference operator $\mathcal{D}_{y}$ is defined in a similar way to $\mathcal{D}_{x}$ but for differences and derivatives in the $y$-direction instead of the $x$-direction. The left and right biased velocity states are defined by

$$
\begin{aligned}
& v^{+x}\left(x_{i}+\frac{h_{x}}{2}, y, t^{n}+\tau\right)=\mathcal{D}_{x} v\left(x_{i+1}-\frac{h_{x}}{2}, y, t^{n}+\tau\right), \\
& v^{-x}\left(x_{i}+\frac{h_{x}}{2}, y, t^{n}+\tau\right)=\mathcal{D}_{x} v\left(x_{i}+\frac{h_{x}}{2}, y, t^{n}+\tau\right), \\
& v^{+y}\left(x, y_{j}+\frac{h_{y}}{2}, t^{n}+\tau\right)=\mathcal{D}_{y} v\left(x, y_{j+1}-\frac{h_{y}}{2}, t^{n}+\tau\right), \\
& v^{-y}\left(x, y_{j}+\frac{h_{y}}{2}, t^{n}+\tau\right)=\mathcal{D}_{y} v\left(x, y_{j}+\frac{h_{y}}{2}, t^{n}+\tau\right) .
\end{aligned}
$$

Following the developments in Section 3.1 we are led to

Proposition 4.1. Upwind approximations to the time averaged flux functions (86)-(87) are given by

$$
\begin{aligned}
& \mathcal{F}_{x}^{v}\left(x_{i+\frac{1}{2}}, y_{j}, t^{n}\right) \approx \mathcal{F}_{i+\frac{1}{2}, j}^{v}\left(t^{n}, \Delta t\right)=F^{x}(\Delta t,\{1 /(p+1)\}), \\
& \mathcal{F}_{y}^{v}\left(x_{i}, y_{j+\frac{1}{2}}, t^{n}\right) \approx \mathcal{F}_{i, j+\frac{1}{2}}^{v}\left(t^{n}, \Delta t\right)=F^{y}(\Delta t,\{1 /(p+1)\}), \\
& \mathcal{F}_{x}^{u}\left(x_{i+\frac{1}{2}}, y_{j}, t^{n}\right) \approx \mathcal{F}_{i+\frac{1}{2}, j}^{v}\left(t^{n}, \Delta t\right)=F^{x}(\Delta t,\{1 /((p+1)(p+2))\}), \\
& \mathcal{F}_{y}^{u}\left(x_{i}, y_{j+\frac{1}{2}}, t^{n}\right) \approx \mathcal{F}_{i, j+\frac{1}{2}}^{u}\left(t^{n}, \Delta t\right)=F^{y}(\Delta t,\{1 /((p+1)(p+2))\}),
\end{aligned}
$$

where

$$
\begin{aligned}
& F^{x}\left(\tau,\left\{k_{p}\right\}\right)=\sum_{j=0}^{\infty} \sum_{m=0}^{\infty} \alpha_{j} h_{x}^{2 j}\left\{\frac{k_{m} \tau^{m}}{m !} \partial_{x}^{2 j+1} \mathcal{L}_{m}(u, v)\left(x_{i+\frac{1}{2}}, y_{j}, t^{n}\right)\right. \\
& \left.+\frac{1}{2 c_{x}} \sum_{n=0}^{m}\left(\begin{array}{c}
m \\
n
\end{array}\right) \frac{k_{m-n} \tau^{m-n}}{m !}\left(\frac{h_{x}}{2}\right)^{n} \partial_{x}^{n+2 j}\left[(-1)^{n} \mathcal{L}_{m-n+1}(v, u)\left(x_{i+1}, y_{j}, t^{n}\right)-\mathcal{L}_{m-n+1}(v, u)\left(\mathbf{x}_{\mathbf{i}}, t^{n}\right)\right]\right\}, \\
& F^{y}\left(\tau,\left\{k_{p}\right\}\right)=\sum_{j=0}^{\infty} \sum_{m=0}^{\infty} \alpha_{j} h_{y}^{2 j}\left\{\frac{k_{m} \tau^{m}}{m !} \partial_{y}^{2 j+1} \mathcal{L}_{m}(u, v)\left(x_{i}, y_{j+\frac{1}{2}}, t^{n}\right)\right. \\
& \left.+\frac{1}{2 c_{y}} \sum_{n=0}^{m}\left(\begin{array}{c}
m \\
n
\end{array}\right) \frac{k_{m-n} \tau^{m-n}}{m !}\left(\frac{h_{y}}{2}\right)^{n} \partial_{y}^{n+2 j}\left[(-1)^{n} \mathcal{L}_{m-n+1}(v, u)\left(x_{i}, y_{j+1}, t^{n}\right)-\mathcal{L}_{m-n+1}(v, u)\left(\mathbf{x}_{\mathbf{i}}, t^{n}\right)\right]\right\} .
\end{aligned}
$$

Note that the operator $\mathcal{L}_{m}(u, v)$ in (92)-(93) is defined by (36), with $L$ given by (82). The first few terms in $F^{x}\left(\tau,\left\{k_{p}\right\}\right)$ and $F^{y}\left(\tau,\left\{k_{p}\right\}\right)$ are given by

$$
\begin{aligned}
F^{x}\left(\tau,\left\{k_{p}\right\}\right) & =k_{0} \frac{\partial u}{\partial x}\left(x_{i+\frac{1}{2}}, y_{j}, t^{n}\right)+\frac{k_{0}}{2 c_{x}} \Delta_{+x} v\left(\mathbf{x}_{\mathbf{i}}, t^{n}\right)+k_{1} \tau \frac{\partial v}{\partial x}\left(x_{i+\frac{1}{2}}, y_{j}, t^{n}\right) \\
& +\frac{k_{1} \tau}{2 c_{x}} \Delta_{+x} L u\left(\mathbf{x}_{\mathbf{i}}, t^{n}\right)-\frac{k_{0} h_{x}}{2 c_{x}} \mathcal{A}_{+x} \frac{\partial v}{\partial x}\left(\mathbf{x}_{\mathbf{i}}, t^{n}\right)+\mathcal{O}\left(\left(h_{x}+\Delta t\right)^{2}\right), \\
F^{y}\left(\tau,\left\{k_{p}\right\}\right) & =k_{0} \frac{\partial u}{\partial y}\left(x_{i}, y_{j+\frac{1}{2}}, t^{n}\right)+\frac{k_{0}}{2 c_{y}} \Delta_{+y} v\left(\mathbf{x}_{\mathbf{i}}, t^{n}\right)+k_{1} \tau \frac{\partial v}{\partial y}\left(x_{i}, y_{j+\frac{1}{2}}, t^{n}\right) \\
& +\frac{k_{1} \tau}{2 c_{y}} \Delta_{+y} L u\left(\mathbf{x}_{\mathbf{i}}, t^{n}\right)-\frac{k_{0} h_{y}}{2 c_{y}} \mathcal{A}_{+y} \frac{\partial v}{\partial y}\left(\mathbf{x}_{\mathbf{i}}, t^{n}\right)+\mathcal{O}\left(\left(h_{y}+\Delta t\right)^{2}\right) .
\end{aligned}
$$

As in one-dimension, Gaussian quadrature (44) can be used instead of exact integration in time which results in the approximations

$$
\begin{aligned}
& \mathcal{F}_{x}^{v}\left(x_{i+\frac{1}{2}}, y_{j}, t^{n}\right) \approx \mathcal{F}_{i+\frac{1}{2}, j}^{v}\left(t^{n}, \Delta t\right)=\sum_{j=1}^{M} b_{j} F^{x}\left(\xi_{j} \Delta t,\{1\}\right), \\
& \mathcal{F}_{y}^{u}\left(x_{i}, y_{j+\frac{1}{2}}, t^{n}\right) \approx \mathcal{F}_{i, j+\frac{1}{2}}^{u}\left(t^{n}, \Delta t\right)=\sum_{j=1}^{M} d_{j} F^{y}\left(\xi_{j} \Delta t,\{1\}\right),
\end{aligned}
$$

with similar expressions for $\mathcal{F}_{y}^{v}\left(x_{i}, y_{j+\frac{1}{2}}, t^{n}\right)$ and $\mathcal{F}_{x}^{u}\left(x_{i+\frac{1}{2}}, y_{j}, t^{n}\right)$. The fully discrete scheme is then given by

$$
\begin{aligned}
& v_{\mathbf{i}}^{n+1}=v_{\mathbf{i}}^{n}+c_{x}^{2} \Delta t D_{+x}\left[\mathcal{F}_{i-\frac{1}{2}, j}^{v}\left(t^{n}, \Delta t\right)\right]+c_{y}^{2} \Delta t D_{+y}\left[\mathcal{F}_{i, j-\frac{1}{2}}^{v}\left(t^{n}, \Delta t\right)\right], \\
& u_{\mathbf{i}}^{n+1}=u_{\mathbf{i}}^{n}+\Delta t v_{\mathbf{i}}^{n}+c_{x}^{2} \Delta t^{2} D_{+x}\left[\mathcal{F}_{i-\frac{1}{2}, j}^{u}\left(t^{n}, \Delta t\right)\right]+c_{y}^{2} \Delta t^{2} D_{+y}\left[\mathcal{F}_{i, j-\frac{1}{2}}^{u}\left(t^{n}, \Delta t\right)\right] .
\end{aligned}
$$




\subsection{A first-order accurate scheme in two dimensions}

A first-order accurate upwind scheme is defined using

$$
\begin{aligned}
& F^{x}\left(\Delta t,\left\{k_{p}\right\}\right) \approx k_{0} \frac{\partial u}{\partial x}\left(x_{i+\frac{1}{2}}, y_{j}, t^{n}\right)+\frac{k_{0}}{2 c_{x}} \Delta_{+x} v\left(\mathbf{x}_{\mathbf{i}}, t^{n}\right), \\
& F^{y}\left(\Delta t,\left\{k_{p}\right\}\right) \approx k_{0} \frac{\partial u}{\partial y}\left(x_{i}, y_{j+\frac{1}{2}}, t^{n}\right)+\frac{k_{0}}{2 c_{y}} \Delta_{+y} v\left(\mathbf{x}_{\mathbf{i}}, t^{n}\right),
\end{aligned}
$$

with the approximations

$$
\begin{aligned}
& F_{i+1 / 2, j}^{x}\left(\Delta t, k_{0}\right) \equiv k_{0} D_{+x} u_{\mathbf{i}}^{n}+\frac{k_{0}}{2 c_{x}} \Delta_{+x} v_{\mathbf{i}}^{n}, \\
& F_{i, j+1 / 2}^{y}\left(\Delta t, k_{0}\right) \equiv k_{0} D_{+y} u_{\mathbf{i}}^{n}+\frac{k_{0}}{2 c_{y}} \Delta_{+y} v_{\mathbf{i}}^{n} .
\end{aligned}
$$

The scheme can be efficiently evaluated using Gaussian quadrature in time with $M=1$ and quadrature node $\xi_{1}=\frac{1}{2}$ and $b_{1}=1, d_{1}=\frac{1}{2}$, to give

$$
\begin{aligned}
F_{i-\frac{1}{2}, j}^{x, 1} & =F_{i-\frac{1}{2}, j}^{x}\left(\xi_{1} \Delta t, k_{0}=1\right), \quad F_{i, j-\frac{1}{2}}^{y, 1}=F_{i, j-\frac{1}{2}}^{y}\left(\xi_{1} \Delta t, k_{0}=1\right), \\
g_{\mathbf{i}} & =\Delta t\left(c_{x}^{2} D_{+x} F_{i-\frac{1}{2}, j}^{x, 1}+c_{y}^{2} D_{+y} F_{i, j-\frac{1}{2}}^{y, 1}\right), \\
v_{\mathbf{i}}^{n+1} & =v_{\mathbf{i}}^{n}+g_{\mathbf{i}}, \\
u_{\mathbf{i}}^{n+1} & =u_{\mathbf{i}}^{n}+\Delta t v_{\mathbf{i}}^{n}+\frac{\Delta t}{2} g_{\mathbf{i}} .
\end{aligned}
$$

Written out in detail, the scheme is (denoted by UW1-2D)

$$
\begin{aligned}
v_{\mathbf{i}}^{n+1} & =v_{\mathbf{i}}^{n}+\Delta t L_{2 h} u_{\mathbf{i}}^{n}+\frac{\Delta t}{2}\left(c_{x} h_{x} D_{+x} D_{-x}+c_{y} h_{y} D_{+y} D_{-y}\right) v_{\mathbf{i}}^{n}, \\
u_{\mathbf{i}}^{n+1} & =u_{\mathbf{i}}^{n}+\Delta t v_{\mathbf{i}}^{n}+\frac{\Delta t^{2}}{2} L_{2 h} u_{\mathbf{i}}^{n}+\frac{\Delta t^{2}}{4}\left(c_{x} h_{x} D_{+x} D_{-x}+c_{y} h_{y} D_{+y} D_{-y}\right) v_{\mathbf{i}}^{n}, \\
L_{2 h} & \equiv c_{x}^{2} D_{+x} D_{-x}+c_{y}^{2} D_{+y} D_{-y},
\end{aligned}
$$

where $L_{2 h}$ is a second order accurate approximation to the operator $L$.

Lemma 4.2. The scheme UW1-2D (98)-(99) is first-order accurate and stable provided

$$
\Delta t \leq\left[\frac{c_{x}}{h_{x}}+\frac{c_{y}}{h_{y}}\right]^{-1}
$$

Proof A normal mode stability analysis of the scheme is carried out by seeking solutions of the form

$$
\left[\begin{array}{l}
u_{\mu, \nu}^{n} \\
v_{\mu, \nu}^{n}
\end{array}\right]=z^{n}\left[\begin{array}{l}
\hat{u} \\
\hat{v}
\end{array}\right] e^{2 \pi i\left(k_{x} x_{\mu}+k_{y} y_{\nu}\right)},
$$

This ansatz leads to the eigenvalue problem

$$
\left[\begin{array}{cc}
1-z-\frac{\lambda_{x}^{2}}{2} \hat{\xi}_{x}^{2}-\frac{\lambda_{y}^{2}}{2} \hat{\xi}_{y}^{2} & \Delta t\left(1-\frac{\lambda_{x}}{4} \hat{\xi}_{x}^{2}-\frac{\lambda_{y}}{4} \hat{\xi}_{y}^{2}\right) \\
-\frac{1}{\Delta t}\left(\lambda_{x}^{2} \hat{\xi}_{x}^{2}+\lambda_{y}^{2} \hat{\xi}_{y}^{2}\right) & 1-z-\frac{\lambda_{x}}{2} \hat{\xi}_{x}^{2}-\frac{\lambda_{y}}{2} \hat{\xi}_{y}^{2}
\end{array}\right]\left[\begin{array}{c}
\hat{u} \\
\hat{v}
\end{array}\right]=\left[\begin{array}{l}
0 \\
0
\end{array}\right]
$$

Here $\xi_{x}=2 \pi k_{x} / N_{x}$ with $\xi_{x} \in[-\pi, \pi], \xi_{y}=2 \pi k_{y} / N_{y}$ with $\xi_{y} \in[-\pi, \pi], \hat{\xi}_{x}^{2}=4 \sin ^{2}\left(\xi_{x} / 2\right)$ and $\hat{\xi}_{y}^{2}=$ $4 \sin ^{2}\left(\xi_{y} / 2\right)$. The two eigenvalues are

$$
\begin{aligned}
z_{ \pm} & =1-b \pm \sqrt{b^{2}-a}, \\
a & =\lambda_{x}^{2} \hat{\xi}_{x}^{2}+\lambda_{y}^{2} \hat{\xi}_{y}^{2}, \quad b=\frac{1}{4}\left(a+\lambda_{x} \hat{\xi}_{x}^{2}+\lambda_{y} \hat{\xi}_{y}^{2}\right) .
\end{aligned}
$$


Note that $z_{ \pm}$should approximate the exact solution $e^{ \pm i \omega \Delta t}$ where $\omega \Delta t=\sqrt{\lambda_{x}^{2} \xi_{x}^{2}+\lambda_{y}^{2} \xi_{y}^{2}}$. Expanding $z_{ \pm}$for small $\left|\xi_{x}\right|$ and $\left|\xi_{y}\right|$ gives

$$
\begin{aligned}
z_{ \pm} & =1 \pm i \sqrt{\lambda_{x}^{2} \xi_{x}^{2}+\lambda_{y}^{2} \xi_{y}^{2}}-\frac{\lambda_{x}}{4}\left(\lambda_{x}+1\right) \xi_{x}^{2}-\frac{\lambda_{y}}{4}\left(\lambda_{y}+1\right) \xi_{y}^{2}+\mathcal{O}\left(\left(\xi_{x}+\xi_{y}\right)^{3}\right) \\
& =e^{ \pm i \omega \Delta t}+\mathcal{O}\left(\left(\xi_{x}+\xi_{y}\right)^{2}\right)
\end{aligned}
$$

This shows that the scheme is first-order accurate. To determine the stability bounds consider the two cases. Case 1: when $b^{2}<a,\left|z_{ \pm}\right|^{2}=1+a-2 b$. Stability requires $\left|z_{ \pm}\right| \leq 1$, or

$$
\lambda_{x}\left(1-\lambda_{x}\right) \hat{\xi}_{x}^{2}+\lambda_{y}\left(1-\lambda_{y}\right) \hat{\xi}_{y}^{2} \geq 0
$$

and this is satisfied provided $\lambda_{x} \leq 1$ and $\lambda_{y} \leq 1$. Case 2: when $b^{2} \geq a,\left|z_{ \pm}\right| \leq 1$ implies

$$
-1 \leq 1-b \pm \sqrt{b^{2}-a} \leq 1
$$

which leads to the condition $4-4 b+a \geq 0$ or

$$
\lambda_{x} \hat{\xi}_{x}^{2}+\lambda_{y} \hat{\xi}_{y}^{2} \leq 4 .
$$

Since $\hat{\xi}_{x}^{2} \leq 4$ and $\hat{\xi}_{y}^{2} \leq 4$ it follows that for stability

$$
\lambda_{x}+\lambda_{y} \leq 1,
$$

which leads to the time step restriction (101). This condition is more restrictive than that for Case 1 and thus is the required stability condition. Note that as in one-dimension, the most unstable modes are the highest frequency modes, $\xi_{x}= \pm \pi$ and $\xi_{y}= \pm \pi$.

The modified equation for the two-dimensional first-order scheme UW1-2D is found to be

$$
\begin{aligned}
\frac{\partial^{2} U}{\partial t^{2}} & =c_{x}^{2} \frac{\partial^{2} U}{\partial x^{2}}+c_{y}^{2} \frac{\partial^{2} U}{\partial y^{2}}+\frac{c_{x} h_{x}}{2}\left(1-\lambda_{x}\right) \frac{\partial^{3} U}{\partial t \partial x^{2}}+\frac{c_{y} h_{y}}{2}\left(1-\lambda_{y}\right) \frac{\partial^{3} U}{\partial t \partial y^{2}}+\frac{c_{x}^{2} h_{x}^{2}}{12}\left(1-3 \lambda_{x}+2 \lambda_{x}^{2}\right) \frac{\partial^{4} U}{\partial x^{4}} \\
& +\frac{c_{y}^{2} h_{y}^{2}}{12}\left(1-3 \lambda_{y}+2 \lambda_{y}^{2}\right) \frac{\partial^{4} U}{\partial y^{4}}+\frac{h_{x} h_{y} c_{x} c_{y}}{12}\left(4 \lambda_{x} \lambda_{y}-3 \lambda_{x}-3 \lambda_{y}\right) \frac{\partial^{4} U}{\partial x^{2} \partial y^{2}}+\mathcal{O}\left(\left(\Delta t+h_{x}+h_{y}\right)^{3}\right),
\end{aligned}
$$

with $V$ satisfying the same equation. The first set of error terms in (102) (those with coefficients proportional to $h_{x}$ or $h_{y}$ ) are the dissipation terms (provided $\lambda_{x} \leq 1$ and $\lambda_{y} \leq 1$ ). The subsequent terms (with coefficients proportional to $h_{x}^{2}, h_{x} h_{y}$ or $h_{y}^{2}$ ) are dispersion terms.

\subsection{A second-order accurate scheme and a high-resolution scheme in two dimensions}

Second-order accurate and high-resolution schemes in two dimensions can be defined by keeping the first five terms in (94) and (95),

$$
\begin{aligned}
F^{x}\left(\Delta t,\left\{k_{p}\right\}\right) & \approx k_{0} \frac{\partial u}{\partial x}\left(x_{i+\frac{1}{2}}, y_{j}, t^{n}\right)+\frac{k_{0}}{2 c_{x}} \Delta_{+x} v\left(\mathbf{x}_{\mathbf{i}}, t^{n}\right) \\
& +k_{1} \Delta t \frac{\partial v}{\partial x}\left(x_{i+\frac{1}{2}}, y_{j}, t^{n}\right)+\frac{k_{1} \Delta t}{2 c_{x}} \Delta_{+x} L u\left(\mathbf{x}_{\mathbf{i}}, t^{n}\right)-\frac{k_{0} h_{x}}{2 c_{x}} \mathcal{A}_{+x} \frac{\partial v}{\partial x}\left(\mathbf{x}_{\mathbf{i}}, t^{n}\right), \\
F^{y}\left(\Delta t,\left\{k_{p}\right\}\right) & \approx k_{0} \frac{\partial u}{\partial y}\left(x_{i}, y_{j+\frac{1}{2}}, t^{n}\right)+\frac{k_{0}}{2 c_{y}} \Delta_{+y} v\left(\mathbf{x}_{\mathbf{i}}, t^{n}\right) \\
& +k_{1} \Delta t \frac{\partial v}{\partial y}\left(x_{i}, y_{j+\frac{1}{2}}, t^{n}\right)+\frac{k_{1} \Delta t}{2 c_{y}} \Delta_{+y} L u\left(\mathbf{x}_{\mathbf{i}}, t^{n}\right)-\frac{k_{0} h_{y}}{2 c_{y}} \mathcal{A}_{+y} \frac{\partial v}{\partial y}\left(\mathbf{x}_{\mathbf{i}}, t^{n}\right),
\end{aligned}
$$

and approximating these with

$$
\begin{aligned}
F_{i+1 / 2, j}^{x}\left(\Delta t, k_{0}, k_{1}\right) & \equiv k_{0} D_{+x} u_{\mathbf{i}}^{n}+\frac{k_{0}}{2 c_{x}} \Delta_{+x} v_{\mathbf{i}}^{n} \\
& +k_{1} \Delta t D_{+x} v_{\mathbf{i}}^{n}+\frac{k_{1} \Delta t}{2 c_{x}} \Delta_{+x} L_{2 h} u_{\mathbf{i}}^{n}-\frac{k_{0} h_{x}}{2 c_{x}} \mathcal{A}_{+x} S_{L}\left(D_{-x} v_{\mathbf{i}}^{n}, D_{+x} v_{\mathbf{i}}^{n}\right), \\
F_{i, j+1 / 2}^{y}\left(\Delta t, k_{0}, k_{1}\right) & \equiv k_{0} D_{+y} u_{\mathbf{i}}^{n}+\frac{k_{0}}{2 c_{y}} \Delta_{+y} v_{\mathbf{i}}^{n} \\
& +k_{1} \Delta t D_{+y} v_{\mathbf{i}}^{n}+\frac{k_{1} \Delta t}{2 c_{y}} \Delta_{+y} L_{2 h} u_{\mathbf{i}}^{n}-\frac{k_{0} h_{y}}{2 c_{y}} \mathcal{A}_{+y} S_{L}\left(D_{-y} v_{\mathbf{i}}^{n}, D_{+y} v_{\mathbf{i}}^{n}\right) .
\end{aligned}
$$


The unlimited second-order accurate scheme (denoted by UW2-2D) uses $S_{L}(a, b)=(a+b) / 2$ while the highresolution scheme (denoted by HR2-2D) uses $S_{L}(a, b)=\operatorname{MinMod}(a, b)$ (equation 59). The approximation can be efficiently evaluated using Gaussian quadrature in time with $M=1$ and quadrature node $\xi_{1}=\frac{1}{2}$ $\left(b_{1}=1, d_{1}=\frac{1}{2}\right)$ using

$$
\begin{aligned}
F_{i-\frac{1}{2}, j}^{x, 1} & =F_{i-\frac{1}{2}, j}^{x}\left(\xi_{1} \Delta t,\{1\}\right), \quad F_{i, j-\frac{1}{2}}^{y, 1}=F_{i, j-\frac{1}{2}}^{y}\left(\xi_{1} \Delta t,\{1\}\right), \\
g_{\mathbf{i}} & =\Delta t\left(c_{x}^{2} D_{+x} F_{i-\frac{1}{2}, j}^{x, 1}+c_{y}^{2} D_{+y} F_{i, j-\frac{1}{2}}^{y, 1}\right), \\
v_{\mathbf{i}}^{n+1} & =v_{\mathbf{i}}^{n}+g_{\mathbf{i}}, \\
u_{\mathbf{i}}^{n+1} & =u_{\mathbf{i}}^{n}+\Delta t v_{\mathbf{i}}^{n}+\frac{\Delta t}{2} g_{\mathbf{i}} .
\end{aligned}
$$

Lemma 4.3. The unlimited scheme UW2-2D (103)-(106) (with $\left.S_{L}(a, b)=(a+b) / 2\right)$ is second-order accurate and stable provided

$$
\Delta t \leq \frac{\frac{1}{2}\left(\tilde{c}_{x}+\tilde{c}_{y}\right)\left[\sqrt{5+\frac{2 \tilde{c}_{x} \tilde{c}_{y}}{\left(\tilde{c}_{x}+\tilde{c}_{y}\right)^{2}}}-1\right]}{\tilde{c}_{x}^{2}+\tilde{c}_{y}^{2}},
$$

where $\tilde{c}_{x}=c_{x} / h_{x}$ and $\tilde{c}_{y}=c_{y} / h_{y}$.

Proof For the second-order accurate unlimited scheme, normal mode analysis leads to the eigenvalue problem,

where

$$
\left[\begin{array}{cc}
1-z-\frac{1}{2} \gamma+\frac{1}{8} \eta & \Delta t\left(1-\frac{1}{4} \gamma-\frac{1}{16} \zeta\right) \\
-\frac{1}{\Delta t}\left(\gamma-\frac{1}{4} \eta\right) & 1-z-\frac{1}{2} \gamma-\frac{1}{8} \zeta
\end{array}\right]\left[\begin{array}{l}
\hat{u} \\
\hat{v}
\end{array}\right]=\left[\begin{array}{l}
0 \\
0
\end{array}\right]
$$

$$
\gamma \equiv \lambda_{x}^{2} \hat{\xi}_{x}^{2}+\lambda_{y}^{2} \hat{\xi}_{y}^{2}, \quad \delta \equiv \lambda_{x} \hat{\xi}_{x}^{2}+\lambda_{y} \hat{\xi}_{y}^{2}, \quad \eta \equiv \delta \gamma, \quad \zeta \equiv \lambda_{x} \hat{\xi}_{x}^{4}+\lambda_{y} \hat{\xi}_{y}^{4}
$$

The two eigenvalues $z=z_{ \pm}$are given by

$$
\begin{gathered}
z_{ \pm}=1-b \pm \sqrt{b^{2}-a} \\
a=\gamma\left(1-\frac{1}{4} \delta\right), \quad b=\frac{1}{2}\left(\gamma-\frac{1}{8} \eta+\frac{1}{8} \zeta\right) .
\end{gathered}
$$

Expanding $z_{ \pm}$for small $\left|\xi_{x}\right|$ and $\left|\xi_{y}\right|$ we obtain

$$
\begin{aligned}
z_{ \pm} & =1 \pm i W+\frac{1}{2}(i W)^{2}+\mathcal{O}\left(\left(\xi_{x}+\xi_{y}\right)^{3}\right), \\
& =e^{ \pm i \omega \Delta t}+\mathcal{O}\left(\left(\xi_{x}+\xi_{y}\right)^{3}\right),
\end{aligned}
$$

where $W=\sqrt{\lambda_{x}^{2} \xi_{x}^{2}+\lambda_{y}^{2} \xi_{y}^{2}}$ and $\omega \Delta t=W$. This shows that the scheme is second-order accurate. To analyze the stability of the scheme consider the two cases. Case 1: if $b^{2}<a$ then

$$
\begin{aligned}
\left|z_{ \pm}\right|^{2} & =1+a-2 b \\
& =1-\frac{1}{8}(\eta+\zeta) \leq 1,
\end{aligned}
$$

and the scheme is always stable since $\eta \geq 0$ and $\zeta \geq 0$. Case 2 : if $b^{2} \geq a$ then

$$
-1 \leq 1-b \pm \sqrt{b^{2}-a} \leq 1
$$

which leads to the condition $4-4 b+a \geq 0$ or

$$
\gamma+\frac{1}{4} \zeta \leq 4 .
$$

The left hand side of this last expression is maximized when $\xi_{x}= \pm \pi$ and $\xi_{y}= \pm \pi$ and this leads to the stability condition

$$
\lambda_{x}^{2}+\lambda_{y}^{2}+\lambda_{x}+\lambda_{y} \leq 1 .
$$

In terms of a condition on $\Delta t$ this implies (107). 
The modified equation for $U$ for the scheme UW2-2D is determined to be (the equation for $V$ is the same as that for $U$ ),

$$
\begin{aligned}
\frac{\partial^{2} U}{\partial t^{2}} & =c_{x}^{2} \frac{\partial^{2} U}{\partial x^{2}}+c_{y}^{2} \frac{\partial^{2} U}{\partial y^{2}} \\
& +c_{x}^{2} h_{x}^{2} M_{2}\left(\lambda_{x}\right) \frac{\partial^{4} U}{\partial x^{4}}+c_{y}^{2} h_{y}^{2} M_{2}\left(\lambda_{y}\right) \frac{\partial^{4} U}{\partial y^{4}}+\frac{c_{x} c_{y} h_{x} h_{y}}{12}\left(3 \lambda_{x}+3 \lambda_{y}-2 \lambda_{x} \lambda_{y}\right) \frac{\partial^{4} U}{\partial x^{2} \partial y^{2}} \\
& +c_{x} h_{x}^{3} C_{2}\left(\lambda_{x}^{2}\right) \frac{\partial^{5} U}{\partial t \partial x^{4}}+c_{y} h_{y}^{3} C_{2}\left(\lambda_{y}\right) \frac{\partial^{5} U}{\partial t \partial y^{4}}-\frac{\lambda_{x} \lambda_{y} h_{x} h_{y}}{8}\left(c_{y} h_{x}+c_{x} h_{y}\right) \frac{\partial^{5} U}{\partial t \partial x^{2} \partial y^{2}} \\
& +\mathcal{O}\left(\left(\Delta t+h_{x}+h_{y}\right)^{4}\right),
\end{aligned}
$$

where $M_{2}$ and $C_{2}$ are given by (62). The first set of error terms (108) represent dispersive terms while the second set (109) are the ones that add dissipation.

\subsection{A fourth-order accurate scheme in two dimensions}

A fourth-order accurate upwind scheme is derived following the process developed in previous sections. These are a straightforward extension of the fourth-order accurate scheme in one-dimension defined in Section 3.4. Written out in detail, the fourth-order accurate upwind scheme (denoted by UW4-2D) that uses Gaussian quadrature in time with $M=2$, is given by

$$
\begin{aligned}
v_{\mathbf{i}}^{n+1} & =v_{\mathbf{i}}^{n}+\Delta t G_{\mathbf{i}}\left(k_{0}=1, k_{1}=\frac{1}{2}, k_{2}=\frac{1}{3}, k_{3}=\frac{1}{4}\right), \\
u_{\mathbf{i}}^{n+1} & =u_{\mathbf{i}}^{n}+\Delta t v_{\mathbf{i}}^{n}+\Delta t^{2} G_{\mathbf{i}}\left(k_{0}=\frac{1}{2}, k_{1}=\frac{1}{6}, k_{2}=\frac{1}{12}, k_{3}=\frac{1}{18}\right), \\
G_{\mathbf{i}}\left(k_{p}\right) & =k_{0} L_{4 h} u_{\mathbf{i}}^{n}+k_{1} \Delta t L_{4 h} v_{\mathbf{i}}^{n}+\frac{k_{2}}{2} \Delta t^{2} L_{2 h}^{2} u_{\mathbf{i}}+\frac{k_{3}}{6} \Delta t^{3} L_{2 h}^{2} v_{\mathbf{i}} \\
& +\frac{5 k_{0}}{288}\left(c_{x} h_{x}^{5}\left(D_{+x} D_{-x}\right)^{3}+c_{y} h_{y}^{5}\left(D_{+y} D_{-y}\right)^{3}\right) v_{\mathbf{i}}-\frac{k_{1}}{8} \Delta t \mathcal{M}_{h} u_{\mathbf{i}}+\frac{k_{1}}{24} \Delta t \mathcal{N}_{h} u_{\mathbf{i}} \\
& -\frac{k_{2}}{12} \Delta t^{2} \mathcal{M}_{h} v_{\mathbf{i}}+\frac{k_{2}}{48} \Delta t^{2} \mathcal{N}_{h} v_{\mathbf{i}}+\frac{k_{3}}{12} \Delta t^{3}\left(c_{x} h_{x} D_{+x} D_{-x}+c_{y} h_{y} D_{+y} D_{-y}\right) L_{2 h}^{2} u_{\mathbf{i}} .
\end{aligned}
$$

where

$$
\begin{aligned}
L_{4 h} & \equiv c_{x}^{2} D_{+x} D_{-x}\left(1-\frac{h_{x}^{2}}{12} D_{+x} D_{-x}\right)+c_{y}^{2} D_{+y} D_{-y}\left(1-\frac{h_{y}^{2}}{12} D_{+y} D_{-y}\right), \\
\mathcal{M}_{h} & \equiv\left(c_{x} h_{x}^{3}\left(D_{+x} D_{-x}\right)^{2}+c_{y} h_{y}^{3}\left(D_{+y} D_{-y}\right)^{2}\right) L_{2 h}, \\
\mathcal{N}_{h} & \equiv c_{x} c_{y}\left(c_{y} h_{x}-c_{x} h_{y}\right)\left(D_{+x} D_{-x}\right)\left(D_{+y} D_{-y}\right)\left(h_{x}^{2} D_{+x} D_{-x}-h_{y}^{2} D_{+y} D_{-y}\right) .
\end{aligned}
$$

Note that $L_{4 h}$ is a fourth-order accurate discretization of the operator $L$.

Lemma 4.4. The upwind scheme UW4-2D (110)-(112) is fourth-order accurate and stable provided

$$
\Delta t \leq \frac{\Lambda_{4}^{(2 d)}}{\left[\left(\frac{c_{x}}{h_{x}}\right)^{\sigma}+\left(\frac{c_{y}}{h_{y}}\right)^{\sigma}\right]^{1 / \sigma}},
$$

where $\Lambda_{4}^{(2 d)}=1.075$ and $\sigma=2.175$. Note that this bound is not strict.

Proof The proof of accuracy and stability follows as for the second-order accurate scheme and we are led to the analysis of the two roots $z_{ \pm}=z_{ \pm}\left(\lambda_{x}, \lambda_{y}, \xi_{x}, \xi_{y}\right)$. Expanding $z_{ \pm}$for small $\left|\xi_{x}\right|$ and $\left|\xi_{y}\right|$ yields

$$
\begin{aligned}
z_{ \pm} & =1 \pm i W+\frac{1}{2}(i W)^{2} \pm \frac{1}{3 !}(i W)^{3}+\frac{1}{4 !}(i W)^{4}+\mathcal{O}\left(\left(\xi_{x}+\xi_{y}\right)^{5}\right) \\
& =e^{i \omega \Delta t}+\mathcal{O}\left(\left(\xi_{x}+\xi_{y}\right)^{5}\right)
\end{aligned}
$$




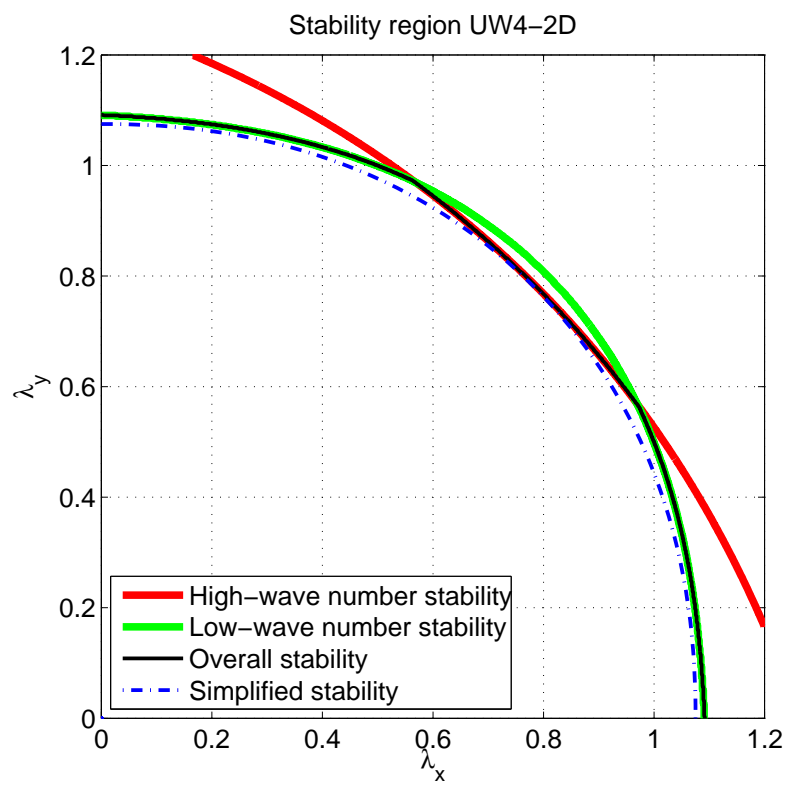

Figure 11: Stability region for the two-dimensional fourth-order accurate scheme UW4-2D. The black curve denotes the boundary of the stability region. The red and blue curves denote the stability regions for high- and low-wave numbers, respectively. The dashed blue curve denotes the simplified curve which is used in practice to determine the time-step $\Delta t$.

where $W=\sqrt{\lambda_{x}^{2} \xi_{x}^{2}+\lambda_{y}^{2} \xi_{y}^{2}}$. This shows that the solution is fourth-order accurate. For stability, we analyze the magnitudes of the roots $z_{ \pm}$. The results are summarized in Figure 11. There are four curves shown in this figure. The black curve, labeled Overall stability, is the level 1 contour of the function

$$
Z\left(\lambda_{x}, \lambda_{y}\right)=\max _{\left|\xi_{x}\right| \leq \pi,\left|\xi_{y}\right| \leq \pi}\left|z_{ \pm}\left(\lambda_{x}, \lambda_{y}, \xi_{x}, \xi_{y}\right)\right| .
$$

To determine this contour, the function $Z$ was evaluated numerically by choosing a fine discretization in $\xi_{x}, \xi_{y}, \lambda_{x}$ and $\lambda_{y}$. The scheme is stable where $Z\left(\lambda_{x}, \lambda_{y}\right) \leq 1$. The green curve, labeled Low-wave number stability, is the stability curve for $\left|\xi_{x}\right| \ll 1$ and $\left|\xi_{y}\right| \ll 1$ (an analytic expression for this curve can be determined from an analysis of the function $Z$ for small $\xi_{x}$ and $\xi_{y}$ ). Recall that in one-dimension, the stability of the scheme is determined for small values of $\xi$. In two-dimensions these low modes determine the stability when $\lambda_{x} \leq \theta \lambda_{y}$ or $\lambda_{y} \leq \theta \lambda_{x}$ where $\theta \approx 0.59$. The red curve, labeled High-wave number stability, is the stability curve for the high-frequency modes $\xi_{x}= \pm \pi$ and $\xi_{y}= \pm \pi$ (the analytic expression for this curve can also be found). The red curve determines the stability bound when $\lambda_{x}>\theta \lambda_{y}$ and $\lambda_{y}>\theta \lambda_{x}$. The blue curve, labeled Simplified stability, was determined by assuming the form

$$
\lambda_{x}^{\sigma}+\lambda_{y}^{\sigma}=b^{\sigma} .
$$

and looking for appropriate values for $b$ and $\sigma$. The choice $b=1.075$ and $\sigma=2.175$ was found to give a curve that is a good approximation to (but falls within) the overall stability curve. These choices of $b$ and $\sigma$ lead to the condition (113).

\section{Accuracy, stability, points per wavelength and remarks}

This section summarizes some of the properties of the upwind schemes which have been developed. The stability bounds, normal mode error formulae and the form of the modified equations for the different schemes are presented. In addition, the accuracy requirements of the schemes in terms of points per wavelength are discussed. The section ends with a few more general remarks.

Figure 12 lists the time-step restriction in terms of the stability bounds, $c \Delta t / h_{x} \leq \Lambda_{p}$, for the different schemes in one-dimension. The figure also shows the stability regions for the two-dimensional schemes with orders of accuracy one, two and four. Note that the fourth-order scheme has a maximum stable time step that is approximately twice that of the second-order scheme (along the axis $\lambda_{x}=\lambda_{y}$ in two-dimensions the stability bounds for the second- and fourth-order schemes are .52 and 1.1 respectively). As a result, we find 


\begin{tabular}{|c|c|c|}
\hline \multicolumn{3}{|c|}{ Stability bounds } \\
\hline $\mathrm{p}$ & scheme & $\Lambda_{p}$ \\
\hline $1 \mathrm{a}$ & $\mathrm{UW} 1 \mathrm{a}$ & .809 \\
1 & $\mathrm{UW} 1$ & 1 \\
2 & $\mathrm{UW} 2$ & .618 \\
4 & $\mathrm{UW} 4$ & 1.09 \\
6 & $\mathrm{UW} 6$ & .95 \\
\hline
\end{tabular}

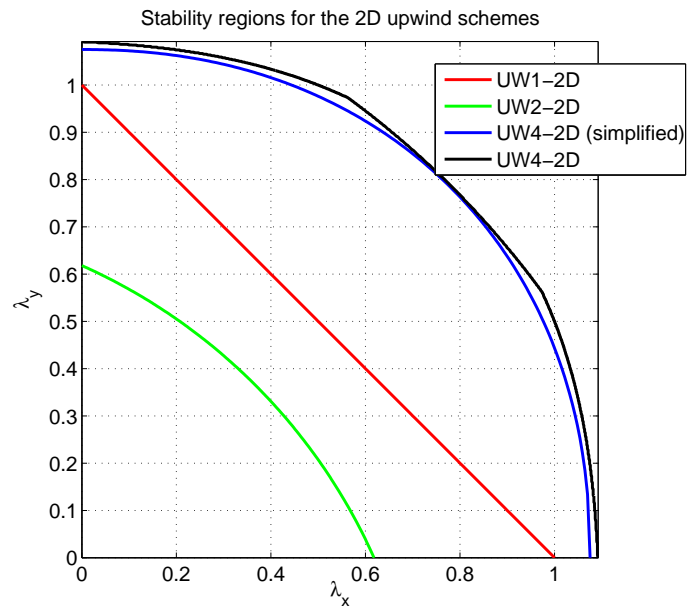

Figure 12: Left: stability bounds, $c \Delta t / h_{x} \leq \Lambda_{p}$, for the one-dimensional upwind schemes with orders of accuracy one to sixth. Right: stability regions for the two-dimensional upwind schemes with orders of accuracy one to four.

that the increased work associated with the fourth-order scheme is nearly offset by the larger allowable time step.

The accuracy of a numerical scheme for wave propagation problems can be described in terms of the number of points per wavelength, $\mathcal{N}_{\lambda}$, that are required to propagate a plane wave solution for a time of $T$ periods and to a relative error of $\epsilon$. This measure of accuracy was originally proposed by Kreiss and Oliger [17] and has subsequently found wide-spread use. The general form of $\mathcal{N}_{\lambda}$ for a $p^{\text {th }}$-order accurate scheme to the first-order wave equation is [18]

$$
\mathcal{N}_{\lambda} \approx K_{p}(T / \epsilon)^{1 / p}
$$

where the parameter $K_{p}$ depends on the details of the scheme. Let $\mathcal{N}_{\lambda}^{(p)}$ denote the number of points per wavelength required by the $p^{\text {th }}$-order accurate upwind schemes for the second-order wave equation. The form (114) also applies to the second-order wave equation (as shown below) and $K_{p}$ can be determined for our upwind schemes. The results are summarized in Figure 13 which not only shows $K_{p}$ for the different schemes but also presents the relationships between the coefficients in the modified equation, the accuracy of the normal-mode analysis eigenvalues $z_{ \pm}$and the number of points-per-wavelength, $\mathcal{N}_{\lambda}^{(p)}$. The results show that the parameter $M_{p}(\lambda)$ that appears in the leading error term in the modified equation also appears in the error of the eigenvalues $z_{ \pm}$and the equations for $\mathcal{N}_{\lambda}^{(p)}$. To understand the reason for these relationships we can look for solutions of the form $e^{i\left(k x-\omega_{p} t\right)}$ to the modified equation (120) (valid for $p=2,4,6$ ), which gives the dispersion relation for the modified equation,

$$
\omega_{p}^{2} \approx c^{2} k^{2}-c^{2} M_{p} h_{x}^{p}(i k)^{p+2}+\mathcal{O}\left(h_{x}^{p+1}\right),
$$

whence,

$$
\omega_{p} \approx \pm c k\left(1+\frac{1}{2} M_{p} h_{x}^{p}(i k)^{p}\right)+\mathcal{O}\left(h_{x}^{p+1}\right) .
$$

Thus, denoting the exact solution as $\omega= \pm c k$, and noting that $\xi=k h_{x}$ and $\lambda=c \Delta t / h_{x}$, it follows that

$$
\begin{aligned}
e^{i \omega_{p} \Delta t}-e^{i \omega \Delta t} & = \pm i c k \Delta t \frac{1}{2} M_{p} h_{x}^{p}(i k)^{p}+\mathcal{O}\left(\Delta t^{2} h_{x}^{p}\right) \\
& = \pm i^{p+1} \frac{\lambda}{2} M_{p} \xi^{p+1}+\mathcal{O}\left(\Delta t^{2} h_{x}^{p}\right) .
\end{aligned}
$$

Solutions to the modified equation should accurately approximate solutions to the difference equations for $\xi \ll 1$ and thus we expect that $z=e^{i \omega_{p} \Delta t}+\mathcal{O}\left(\Delta t h_{x}^{p+1}\right)$. Since $\omega \Delta t=\lambda \xi$, equation (117) thus agrees with (121) (for $p=2,4,6)$. Note that (121) was independently determined from the discrete approximations and normal mode theory. 
The number of points per wavelength is defined from the error in computing the plane wave solution $e^{i k(x \pm c t)}$ over $T$ periods. If $\epsilon$ is the relative error at time $t=n \Delta t$, then from (121),

$$
\epsilon \approx \frac{n \lambda}{2}\left|M_{p} \xi^{p}\right| .
$$

The number of points per wavelength $N_{\lambda}^{(p)}$ is related to $\xi$ by $\xi=2 \pi / N_{\lambda}^{(p)}$. Since the period of the wave is $P=2 \pi /(c k)$ and the number of periods $T$ at time $t$ is $T=t / P$ it follows that

$$
\epsilon \approx \pi\left|M_{p}\right|(2 \pi)^{p} \frac{T}{N_{\lambda}^{(p)}},
$$

which gives (122).

In Figure 14 we plot the parameters $K_{p}(\lambda)$ versus $\lambda$ for $\lambda \in[0,1]$. These results show that the accuracy of the UW1 scheme is poor for small $\lambda$ but very good as $\lambda$ approaches 1 . The accuracy of UW2 improves somewhat as $\lambda$ becomes smaller, while the accuracy of the UW4 and UW6 schemes do not vary much with $\lambda$. Figure 14 also shows plots of $\mathcal{N}_{\lambda}^{(p)}$ versus $\epsilon / T$ for $\lambda=0.5$. The results show the dramatic decrease in $\mathcal{N}_{\lambda}^{(p)}$ for the high-order accurate schemes, especially when $\epsilon / T$ is small (i.e. small error tolerances or long time integrations). For example, with $\epsilon / T=10^{-4}$ the scheme UW1 would require approximately 50,000 points per wavelength to achieve the same accuracy as the scheme UW6 using approximately 15 points per wavelength.

$$
\begin{array}{ll}
\frac{\partial^{2} U}{\partial t^{2}}=c^{2} \frac{\partial^{2} U}{\partial x^{2}}+c^{2} M_{p}(\lambda) h_{x}^{p} \frac{\partial^{p+2} U}{\partial x^{p+2}}+c C_{p}(\lambda) h_{x}^{p+1} \frac{\partial^{p+3} U}{\partial t \partial x^{p+2}}+\mathcal{O}\left(h_{x}^{p+2}\right), & p=2,4,6, \\
z_{ \pm}-e^{ \pm i \lambda \xi}=( \pm i)^{p+1} \frac{\lambda}{2} M_{p}(\lambda) \xi^{p+1}+\mathcal{O}\left(\xi^{p+2}\right), & p=1,2,4,6, \\
\mathcal{N}_{\lambda}^{(p)} \approx K_{p}(T / \epsilon)^{1 / p}, \quad K_{p}=2 \pi\left(\pi\left|M_{p}(\lambda)\right|\right)^{1 / p}, & p=1,2,4,6 .
\end{array}
$$

\begin{tabular}{|c|c|c|}
\hline $\mathrm{p}$ & scheme & $M_{p}(\lambda)$ \\
\hline 1 & $\mathrm{UW} 1$ & $\frac{1}{2}(1-\lambda)$ \\
2 & $\mathrm{UW} 2$ & $\frac{1}{12}\left(1+3 \lambda-\lambda^{2}\right)$ \\
4 & $\mathrm{UW} 4$ & $\frac{1}{2600}\left(-24-135 \lambda+60 \lambda^{2}+45 \lambda^{3}-16 \lambda^{4}\right)$ \\
6 & $\mathrm{UW6}$ & $\frac{1}{1008000}\left(1800+14700 \lambda-4900 \lambda^{2}-7000 \lambda^{3}+2100 \lambda^{4}-197 \lambda^{5}\right)$ \\
\hline
\end{tabular}

Figure 13: The relationship between the coefficients $M_{p}(\lambda)$, the general form of the modified equations (120), the mode analysis solutions $z_{ \pm}$and the number of points per wavelength $\mathcal{N}_{\lambda}^{(p)}$ for the upwind schemes of different orders of accuracy. Note that the modified equation for $p=1$ is the only one not of the form (120).

We conclude this section with some short remarks.

Remark 1: Cache efficiency: The space-time schemes are one-step schemes and can be made very efficient in terms of access to the computer memory and cache. The entire solution can be updated in a single loop, thus passing through the data a single time. We have used automatic code generation to produce optimized routines for the schemes from symbolic representations of the approximations (using the Maple symbolic algebra package).

Remark 2: Storage requirements: The storage required to implement the upwind schemes developed herein is essentially equivalent to that required for the standard C2 scheme (17), even though the additional variable $v$ has been introduced. An efficient implement of the $\mathrm{C} 2$ scheme requires just two time levels to be stored, $u_{i}^{n}$ and $u_{i}^{n-1}$. However, an efficient implementation of the upwind schemes (even in multiple space 

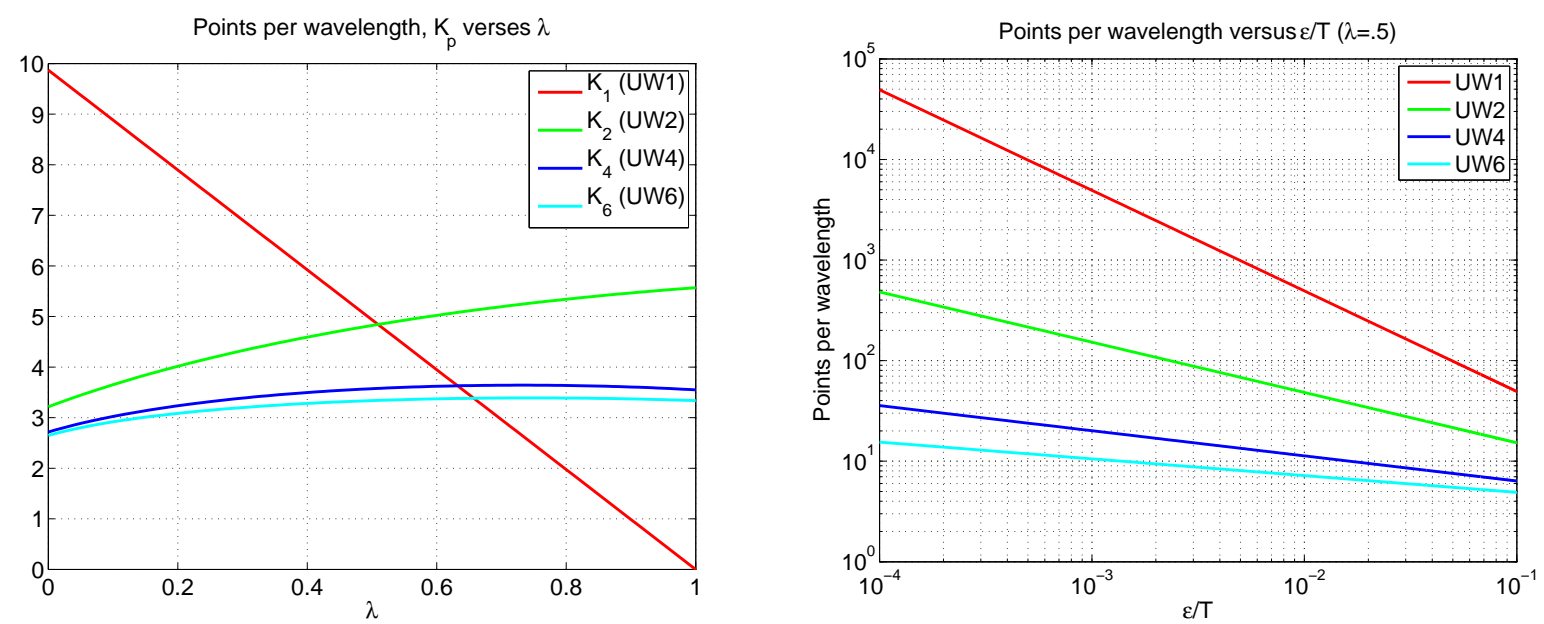

Figure 14: Points per wavelength. Left: the points per wavelength parameters $K_{p}$ as a function of the CFL parameter $\lambda$. Right: the points per wavelength requirements of the different schemes as a function of $\epsilon / T$ for $\lambda=0.5$.

dimensions) only requires storage for $u_{i}^{n}$ and $v_{i}^{n}$, plus some small additional lower-dimensional work space. Therefore the standard and upwind schemes have asymptotically the same storage requirements.

Remark 3: Method of lines: Method-of-lines upwind schemes using Runge-Kutta or multi-step methods can be defined in a straight-forward manner from the developments presented here.

Remark 4: Confirmation of the stability bounds: The analytically derived stability bounds of the oneand two-dimensional schemes were confirmed numerically to within one percent.

Remark 5: Use of the upwind flux: Interestingly, the use of upwind flux is found to be critical to the stability of all but the second-order accurate scheme. In particular, if the upwind flux, formula (31) is replaced by a flux that does not include the second term involving the jump in $v$, then the resulting algorithms, with the exception of the second-order algorithm, will be unconditionally unstable for any time step. The resulting second-order accurate algorithm is neutrally stable with an amplification factor whose magnitude is uniformly one.

\section{Numerical examples}

We now present some numerical results to demonstrate the accuracy and behaviur of the upwind schemes for the second-order wave equation. We begin in Section 6.1 by examining the errors and convergence rates of the schemes in one dimension for a traveling sine wave exact solution. The convergence rates for the more difficult top-hat problem, introduced previously, are presented in Section 6.2. The behavior of the schemes for a smooth two-dimensional surface wave problem with known solution is studied in Section 6.3. Finally in Section 6.4 we show results for a difficult two-dimensional version of the top-hat problem.

Convergence of the approximations will be measured using the $L_{\infty}$-norm (max-norm) and the discrete $L_{1}$-norm. In general, the discrete $L_{p}$-norm of a grid function $u_{\mathbf{i}}$ is defined as

$$
\left\|u_{\mathbf{i}}\right\|_{p}=\left[\frac{1}{N} \sum_{\mathbf{i}}\left|u_{\mathbf{i}}\right|^{p}\right]^{1 / p},
$$

where the sum is taken over all grid points and $N$ is the total number of grid points. In subsequent discussions the terms $L_{\infty}$-norm and $L_{1}$-norm will be used to indicate the corresponding discrete norms.

\subsection{One-dimensional traveling sine wave}

The exact solution of a traveling sine wave, $u(x, t)=\sin (\pi(x-c t))$, can be used to demonstrate the accuracy of the upwind schemes in one dimension. The problem is solved on the periodic interval $[-1,1]$ with $c=1$ and integrated to a final time $t=2$ (one period). For each scheme, the time-step is chosen to be 0.9 times the maximum allowable time-step for that scheme (i.e. a CFL number of 0.9). Figure 15 shows the results of a convergence study for the first-order (UW1), second-order (UW2), fourth-order (UW4), and 
sixth-order (UW6) accurate schemes for this problem for a sequence of meshes with increasing resolution. Results are shown for the $L_{\infty}$-norm (max-norm) of the error. The four scheme are seen to converge as expected. Given that the additional cost per time-step to evaluate the high-order schemes is not much more than the lower order accurate schemes, these results show the clear benefit of using high-order accurate schemes in terms of the effort required to reach a required level of accuracy.
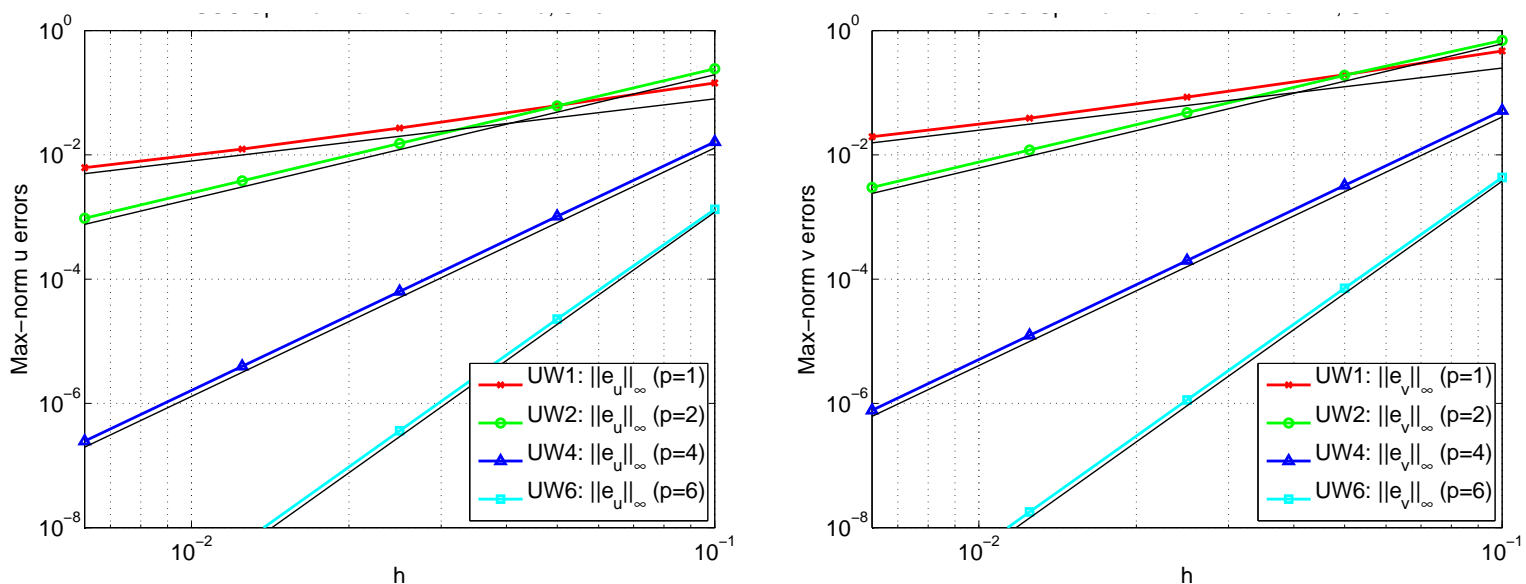

Figure 15: Traveling sine wave: convergence results for the $L_{\infty}$-norm errors at $t=2.0$. Results are presented for the first-, second-, fourth-, and sixth-order accurate schemes. Reference lines of the corresponding order are displayed in black. At left are the results for $u$ and at right the results for $v$.
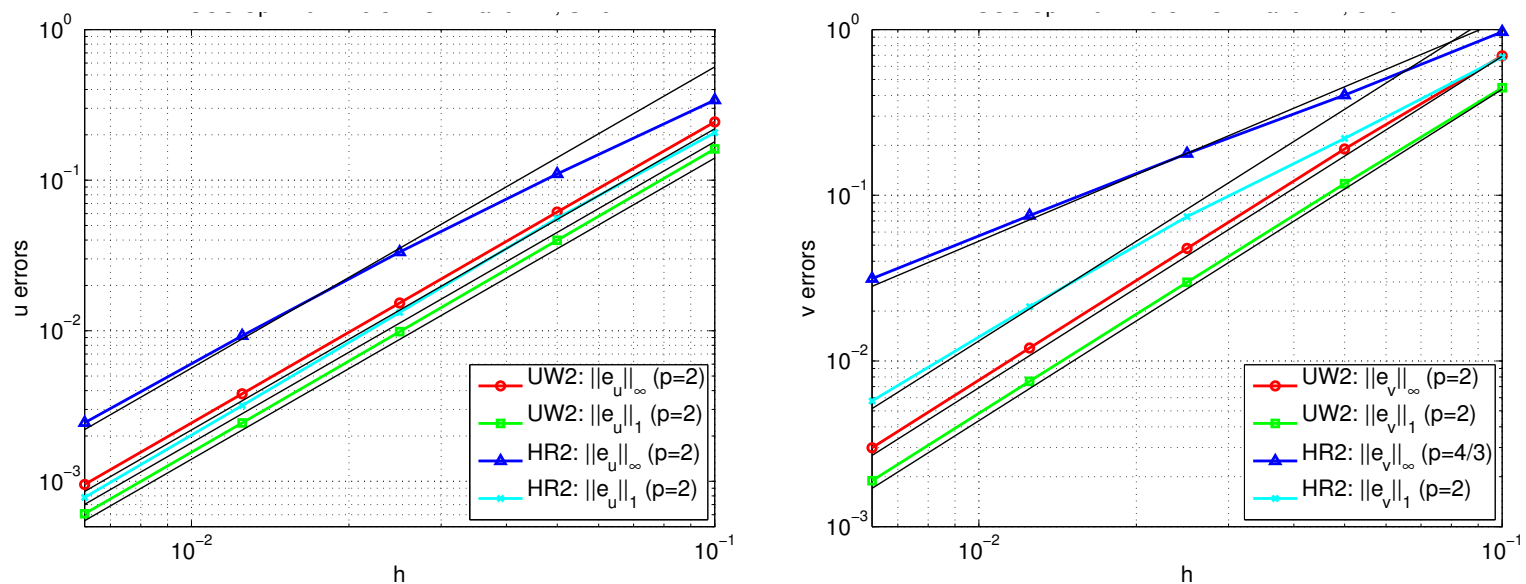

Figure 16: Traveling sine wave: convergence results for the $L_{\infty}$-norm and $L_{1}$-norm errors for the UW2 and HR2 schemes showing the degradation in convergence for the velocity in the HR2 scheme due to the limiter. Left: results for $u$. Right: results for $v$. The $L_{\infty}$-norm errors for $v$ are seen to converge at a rate $p=4 / 3$. All other cases converge at $p=2$.

The behavior of the high-resolution scheme HR2 compared to the second-order accurate scheme UW2 for this problem is presented in Figure 16 where both the max- and $L_{1}$-norms of the error are shown. From the figure it is clear that the second-order scheme converges as expected, but the convergence character of the high-resolution scheme is somewhat more complex. For the high-resolution scheme, $u$ converges at second order for both $L_{1^{-}}$and $L_{\infty}$-norms. However, the velocity $v$ converges as $O\left(h_{x}^{2}\right)$ in the $L_{1}$-norm but the degraded rate of $O\left(h_{x}^{4 / 3}\right)$ for the $L_{\infty}$-norm. This somewhat surprising behavior is the result of the sharp switch introduced by the MinMod limiter and is similar to behavior exhibited by high-resolution schemes for the first-order system [19].

\subsection{One-dimensional top-hat problem}

The top-hat problem was introduced earlier in Section 2. The initial conditions are (13)-(14) and the exact solution is given by (15)-(16). As noted previously, this is a very difficult problem since the solution 
for $u$ does not have a continuous first derivative, while $v$ is defined in terms of Dirac delta functions. The behavior of the discrete solution using all five schemes is shown in Figure 17. This is a rather weak solution
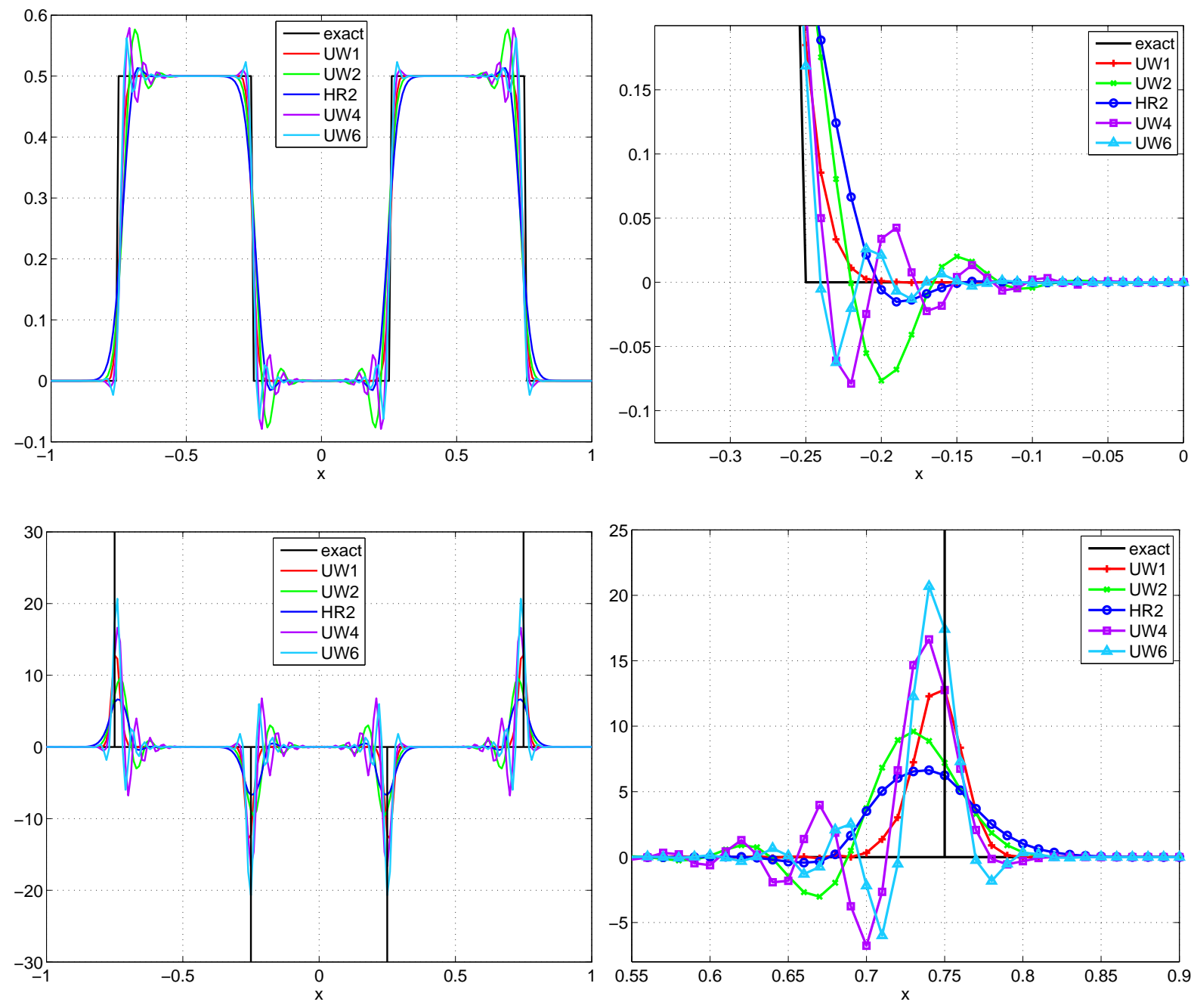

Figure 17: Top-hat: numerical solutions using the first-order (UW1), second-order (UW2), second-order highresolution (HR2), fourth-order (UW4), sixth-order (UW6) schemes. The solutions for $u$ (top) and $v$ (bottom) are shown at $t=0.5$, computed using $N=201$ grid points.

and it is somewhat surprising that a numerical method can provide reasonable approximations in this regime. Nonetheless we see that all methods provide quite good results. The trend from low to high order is clear from the plots. Also clear is the effect of using the limiter in the high-resolution scheme where the oscillations near the discontinuity are significantly suppressed in comparison to the unlimited second order scheme. We would like to draw particular attention however, to the high quality of the results even for the high order schemes. The sixth-order scheme for instance has surprisingly mild oscillations near the discontinuity while capturing the jump with only a few points. Also note that the size of the discrete spike for the Dirac delta function in $v$ is a rather good indicator of the resolving power of the various schemes.

Although the $L_{1}$-norm errors are a good measure of the convergence of $u$, it is not a good measure for the convergence of the delta functions in $v$. Instead we measure the $L_{1}$-norm error in the integral of $v$,

$$
\hat{v}(x, t)=\int_{-\infty}^{x} v(\xi, t) d \xi
$$

which is in some sense a measure of the size and location of the delta functions in $v$. Figure 18 shows the convergence character of the five schemes for $u$ and $\hat{v}$. All the schemes under consideration converge at the rate $O\left(h_{x}^{p / p+1}\right)$ where $p$ is the nominal convergence rate for the scheme for smooth problems. These 

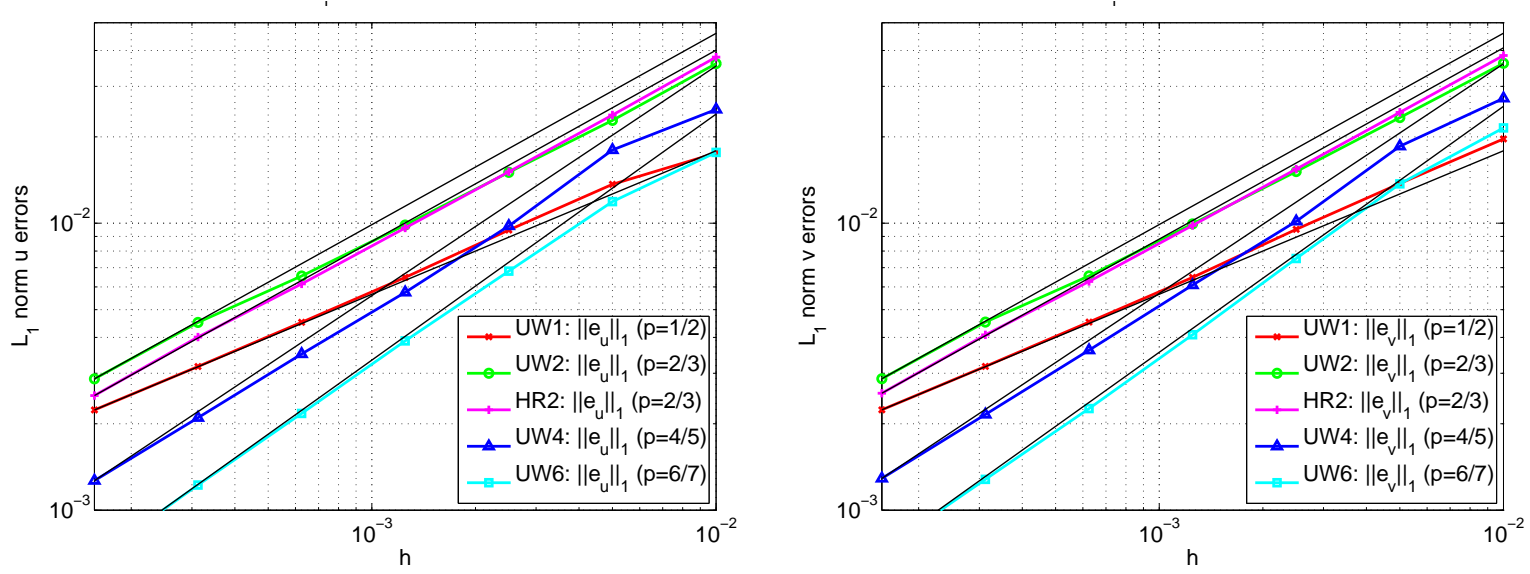

Figure 18: Top-hat: $L_{1}$ convergence of $u$ and $V=\int_{-\infty}^{x} v(\xi, t) d \xi$ at $t=0.5$. The solid black lines are drawn for reference and have slopes $\frac{p}{p+1}$ for $p=1,2,4,6$.

are the expected convergence rates for numerical approximations to the first-order system with jump initial data [19].

\subsection{A two-dimensional surface wave problemn}

Surface waves are an interesting phenomena associated with wave equations and we consider the numerical simulation of such a wave as a verification of our upwind schemes in two-dimensions. Consider the initial boundary value problem for the second-order wave equation in two-dimensions,

$$
\begin{aligned}
\frac{\partial^{2} u}{\partial t^{2}} & =c_{x}^{2} \frac{\partial^{2} u}{\partial x^{2}}+c_{y}^{2} \frac{\partial^{2} u}{\partial y^{2}}, \quad \mathbf{x} \in(-\pi, \pi) \times(-\infty, 0), \\
u(x, y, 0) & =u_{0}(x, y), \quad \frac{\partial u}{\partial t}(x, y, 0)=v_{0}(x, y), \\
\frac{\partial u}{\partial y}(x, 0, t) & =\alpha u(x, 0, t), \quad u(x+2 \pi, y, t)=u(x, y, t),
\end{aligned}
$$

where $\alpha \in \mathbb{R}$. We look for traveling surface wave solutions to these equations of the form $u=e^{\beta y} e^{i(k x-\omega t)}$ which are periodic in $x$ and decay to zero as $y \rightarrow-\infty$. Solutions of this type do exist and are given by

$$
u(x, y, t)=A_{k} e^{\alpha y} \cos \left(k x \pm \omega t+\phi_{k}\right), \quad k=0, \pm 1, \pm 2, \ldots,
$$

where $A_{k}$ and $\phi_{k}$ are constants and where $\omega$ satisfies the dispersion relation $\omega=\sqrt{c_{x}^{2} k^{2}-c_{y}^{2} \alpha^{2}}$. Solutions that remain bounded in time and decay to zero as $y \rightarrow-\infty$ require $0<\alpha \leq\left(c_{x} / c_{y}\right) k$ and $A_{0}=0$ (since the $k=0$ mode with grow exponentially in time if $\alpha \neq 0$ ). The phase velocity of the surface waves (126) is given by

$$
c_{s} \equiv \frac{\omega}{k}=\sqrt{c_{x}^{2}-\frac{c_{y}^{2} \alpha^{2}}{k^{2}}} .
$$

The waves are dispersive with long wave lengths ( $k$ small) propagating more slowly than short wavelengths $(k$ large). Note that mode $k$ becomes stationary if $\alpha=\frac{c_{x}}{c_{y}} k$, while the speed of propagation of short wavelengths approaches $c_{x}$ as $k \rightarrow \infty$.

Equations (123)-(125) are solved numerically for the displacement, $u_{\mathbf{i}}^{n}$, and velocity, $v_{\mathbf{i}}^{n}$, with initial conditions for $u_{\mathbf{i}}^{0}$ and $v_{\mathbf{i}}^{0}$ taken from the exact solution at $t=0$. At $y=0$, boundary conditions are applied to both displacement and velocity. The boundary condition $u_{y}(x, 0, t)=\alpha u(x, 0, t)$ is differentiated with respect to time to yield $v_{y}(x, 0, t)=\alpha v(x, 0, t)$. For the first- and second-order accurate schemes, UW1, UW2, and HR2, the boundary conditions are imposed using a second- and fourth-order accurate approximation to $u_{y}(x, 0, t)=\alpha u(x, 0, t)$,

$$
\begin{aligned}
& \frac{1}{2 h_{y}}\left(u_{i, j+1}^{n}-u_{i, j-1}^{n}\right)=\alpha u_{i, j}^{n}, \\
& \frac{1}{12 h_{x}}\left(u_{i, j-2}^{n}-8 u_{i, j-1}^{n}+8 u_{i, j+1}^{n}-u_{i, j+2}^{n}\right)=\alpha u_{i, j}^{n},
\end{aligned}
$$


where $j$ is the index corresponding to the cell with center on the boundary $y=0$ and $j+1$ and $j+2$ denote the indicies of the first and second ghost cells. Similar equations are used for $v_{i j}^{n}$. Equations (128)-(129) are used to determine the values of $u_{i}^{n}$ on two lines of ghost cells, $u_{i, j+1}$ and $u_{i, j+2}$. Note that the interior equation is solved on the boundary cells $(i, j)$.

For the fourth-order accurate scheme, high-order compatibility conditions are derived by repeated differentiation of the boundary condition with respect to time which yields the conditions $u_{y y y}(x, 0, t)=$ $\alpha u_{y y}(x, 0, t)$ and $v_{y y y}(x, 0, t)=\alpha v_{y y}(x, 0, t)$. The values on three ghost lines for the fourth-order accurate scheme UW4 are determined from a fourth- and sixth-order accurate approximation to $u_{y}(x, 0, t)=\alpha u(x, 0, t)$ together with a second-order accurate approximation to $u_{y y y}(x, 0, t)=\alpha u_{y y}(x, 0, t)$,

$$
\begin{aligned}
& \frac{1}{12 h_{y}}\left(u_{i, j-2}^{n}-8 u_{i, j-1}^{n}+8 u_{i, j+1}^{n}-u_{i, j+2}^{n}\right)=\alpha u_{i, j}^{n}, \\
& \frac{1}{2 h_{y}^{3}}\left(-u_{i, j-2}^{n}+2 u_{i, j-1}^{n}-2 u_{i, j+1}^{n}+u_{i, j+2}^{n}\right)=\frac{\alpha}{12 h_{y}^{2}}\left(-u_{i, j-2}^{n}+16 u_{i, j-1}^{n}-30 u_{i, j}^{n}+16 u_{i, j+1}^{n}-u_{i, j+2}^{n}\right), \\
& \frac{1}{60 h_{y}}\left(-u_{i, j-3}^{n}+9 u_{i, j-2}^{n}-45 u_{i, j-1}^{n}+45 u_{i, j+1}^{n}-9 u_{i, j+2}^{n}+u_{i, j+3}^{n}\right)=\alpha u_{i, j}^{n} .
\end{aligned}
$$

Similar equations are used to obtain the values of $v_{\mathbf{i}}^{n}$ at the ghost points. For practical reasons, the domain is truncated in the $y$-direction to the interval $y \in[-2 \pi, 0]$. The exact solution is imposed at $y=-2 \pi$ so that any possible errors arising from the artificial truncation of the domain are eliminated. To prevent any exponential growth from the $k=0$ mode the approximate solution is projected on the boundary $y=0$ to satisfy a discrete approximation to $\int_{-\pi}^{\pi} u(x, 0, t) d x=0$.

This problem is solved numerically using the parameters $k=1, c_{x}=1 / 2, c_{y}=1, \alpha=0.4$ and $A_{1}=1$. For these parameters, the speed of the surface wave is $c_{s}=0.3$. Numerical approximations are generated using schemes UW1-2D, UW2-2D, HR2-2D and UW4-2D. In each case a CFL number equal to 0.9 is used, (i.e. at $90 \%$ of the maximum stable limit as given in equation (101) for the first-order scheme, equation (107) for the second-order scheme, and equation (113) for the fourth-order scheme). Figure 19 shows contour plots of the solution at $t=5$ for both $u$ and $v$.
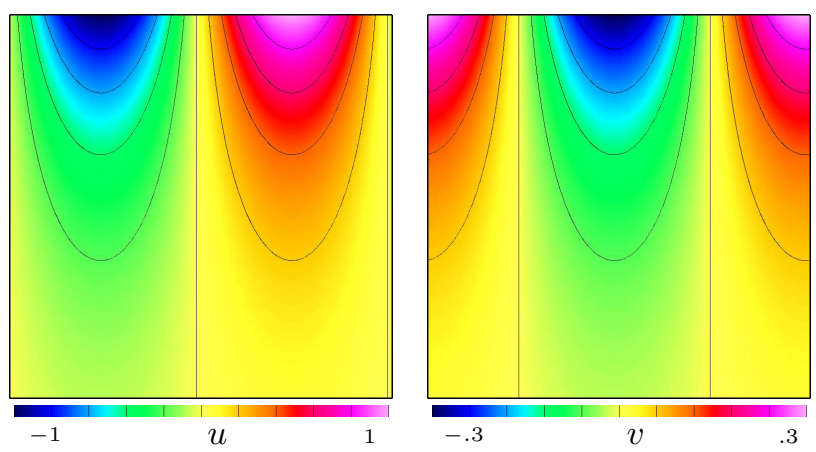

Figure 19: Surface wave solutions of the form (126) for $k=1$ at $t=5$. At left is displacement $u$ and at right the velocity $v$.

Figure 20 shows contours of the error in the computed results for the three methods at $t=5$ using 800 grid cells in the $x$ and $y$ directions. A convergence study of the $L_{\infty}$-norm errors at $t=5$ for the displacement and velocity is shown in Figure 21. The errors in displacement and velocity all converge at the expected rates of $p$ for the $p^{\text {th }}$-order accurate schemes.

Note that in contrast to the case of the traveling sine wave in Section 6.1 when the velocity for the HR2 scheme converged at a degraded rate of $4 / 3$ in the max-norm, in this case the velocity converges at a rate of 2 in the max-norm. The reason for this curious result is traced to the fact that the surface wave is traveling at a different speed, $c_{s}$, than the characteristic wave speed in the $x$-direction, $c_{x}$. Indeed, the $4 / 3$ rate is reproduced for $\alpha=0$ when $c_{s}=c_{x}$. For the case $c_{s}=c_{x}$, the errors generated by the limiter (which are localized near maximuma and minima in the solution) move with the solution. The errors near the maximum or minimum thus accumulate over time, resulting in a degradation to the maximum norm convergence rates. On the other hand when the speeds $c_{s}$ and $c_{x}$ are different, the errors introduced by the limiter do not coincide with previous errors and thus the errors are more spread out; the maximum norm errors are not degraded in this case. 

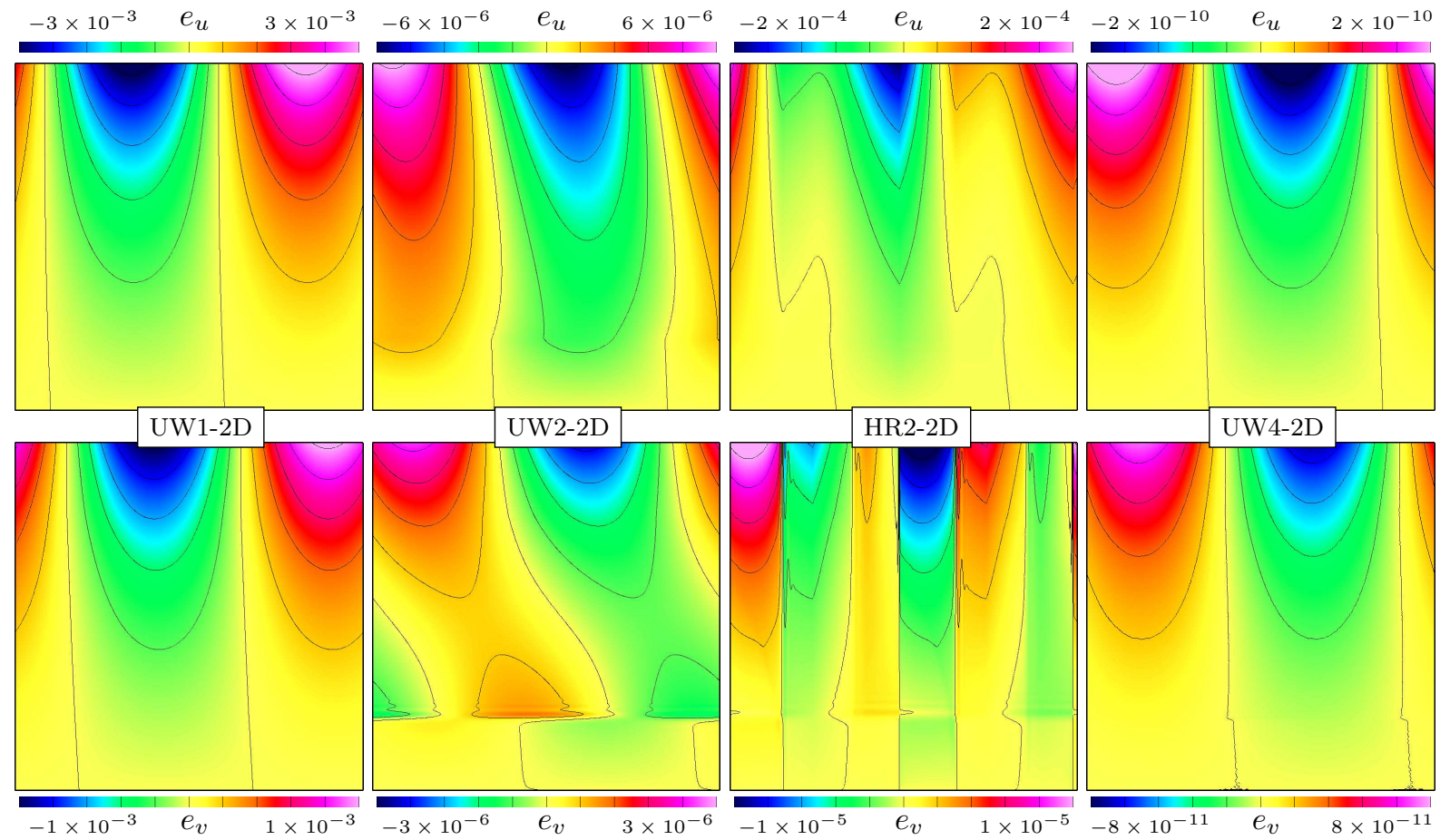

Figure 20: Errors in $u$ and $v$ at $t=5$ using 800 grid cells in the $x$ and $y$ directions. On top are errors in displacement while on bottom are those for velocity. From left to right the results were computed using the first-order scheme, UW1-2D, the second-order scheme, UW2-2D, the high-resolution scheme, HR2-2D, and the fourth-order scheme, UW4-2D. Note the changing scales of the color tables for each contour plot.
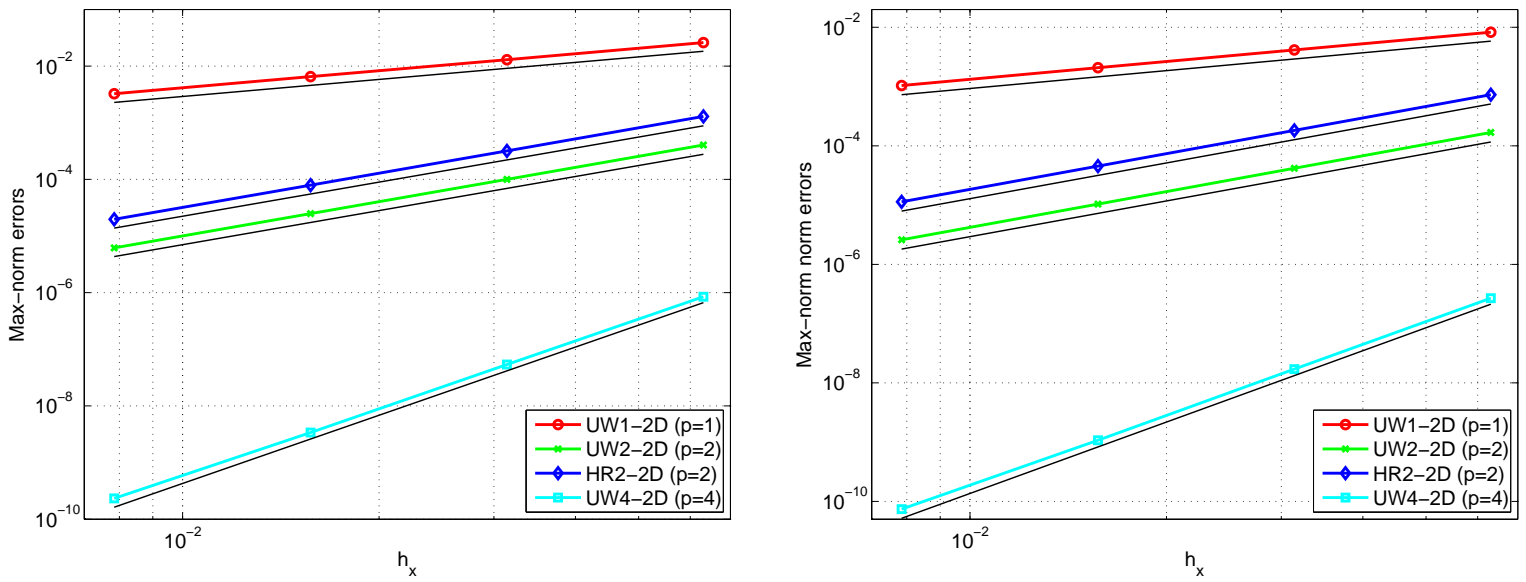

Figure 21: Surface wave: convergence results for the $L_{\infty}$-norm errors in $u$ (left) and $v$ (right) at $t=5$.

\subsection{A top-hat problem in two space dimensions}

As a final example consider the solution to a two-dimensional version of the top-hat problem. We solve the two-dimensional second-order wave equation (81) on the domain $[-\pi, \pi] \times[-2 \pi, 0]$ and take $c_{x}=1 / 2$, $c_{y}=1$. The initial conditions are chosen to be

$$
v(x, y, 0)=0, \quad u(x, y, 0)= \begin{cases}1 & \text { if } x^{2}+(y+2)^{2}<1 \\ 0 & \text { otherwise }\end{cases}
$$

Periodic boundary conditions are applied in the $x$-direction, and a zero normal derivative is applied at the top and bottom boundaries. The numerical approximations to the normal derivative boundary conditions are given as in Section 6.3 with $\alpha=0$. 


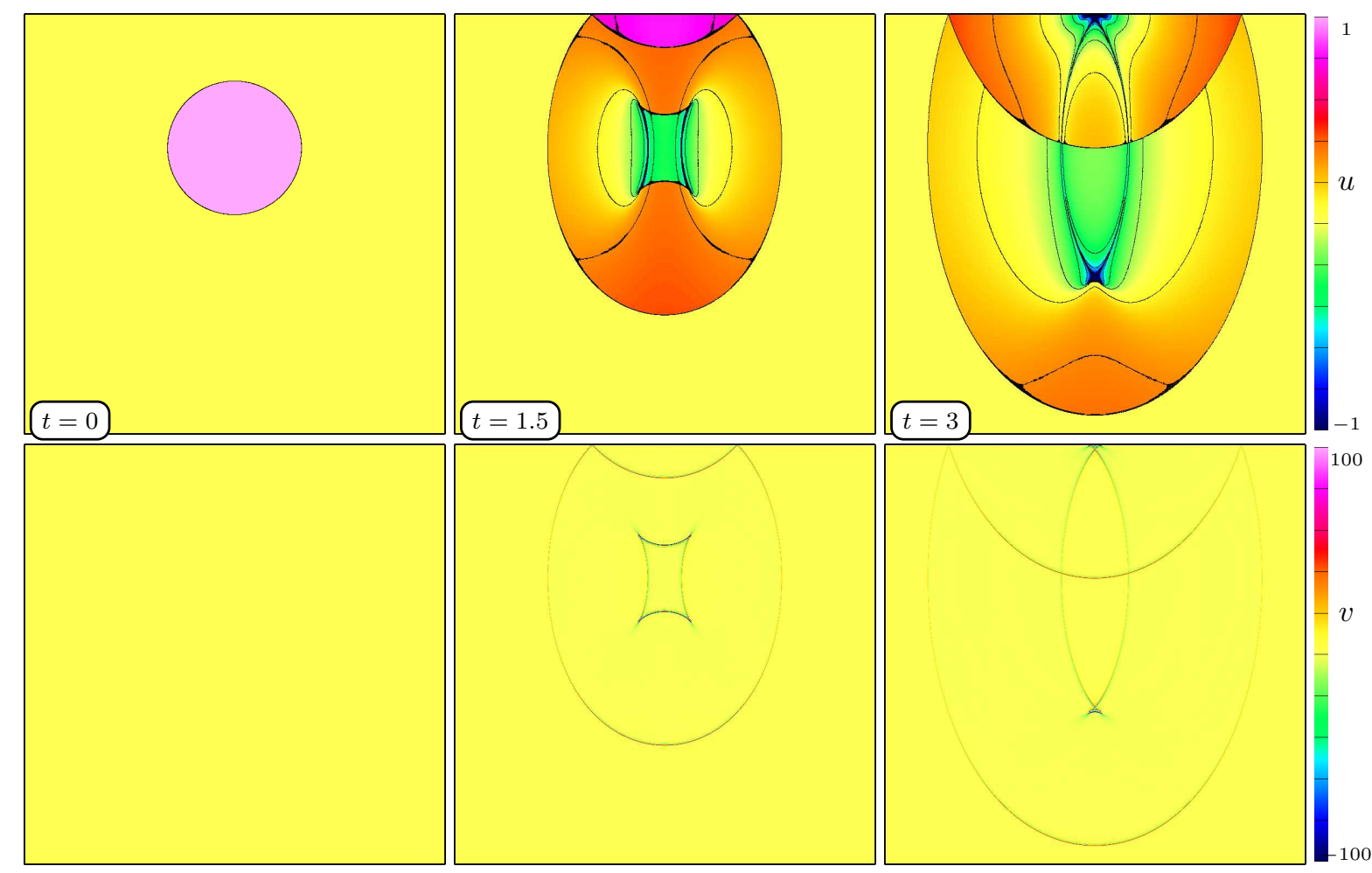

Figure 22: Two-dimensional top-hat: Time evolution of $u$ (top) and $v$ (bottom) using the fourth-order accurate method UW4-2D with 6400 points in each coordinate direction.

Figure 22 shows the computed results using the fourth-order accurate scheme, UW4-2D, at times $t=0$, $t=1.5$, and $t=3.0$. The solution was computed using 6400 points in the $x$ - and $y$-directions. The superimposed black contour lines for the images of $u$ illustrate the remarkably smooth nature of the approximation (contour lines being a sensitive indicator of oscillations in the solution). Note that to present the results more clearly, small regions where $u$ becomes large and negative have been clipped to blue, while both large positive and large negative values of $v$ have been clipped to pink and blue, respectively.

In order to compare the relative merits of the various schemes, Figure 23 shows contours of the displacement for the four schemes, UW1-2D, UW2-2D, HR2-2D and UW4-2D. Results are shown from a coarse grid computation that used 100 cells in each direction and for a slightly finer grid computation that used 200 cells per coordinate direction. Note that these figures use the same scale as the very fine computation in Figure 22. In all cases, the schemes are giving results that are converging toward the very fine grid solution. The figures show the clear benefit of using the high-order accurate upwind schemes. The basic structure of the solution can be seen in the results from the first-order accurate scheme, but the results from the fourthorder accurate scheme show much more detail. There is some evidence of minor oscillations in the UW2-2D and UW4-2D schemes near the solution discontinuities (the HR2-2D seems to suppress these oscillations), but these are consistent with the one-dimensional top-hat results.

Figure 24 shows plots of the displacement and velocity along the top boundary at $y=0$ for the four schemes. These results are from a fine grid computation which used 1600 cells in each direction. This figure shows the much enhanced resolving power of the fourth-order accurate scheme compared to the lower order accurate schemes. Indeed, from a points per wavelength argument (see Section 5) the first- and second-order accurate schemes would require on the order of $1600^{4}$ and $1600^{2}$ grid points respectively in each direction to obtain similar results to the fourth-order accurate scheme. We note that the fine feature near the center of the plot is almost completely lost in results from the first-order accurate scheme. There is a clear trend in improved resolution moving from UW1-2D, to HR2-2D, to UW2-2D and UW4-2D. The HR2-2D scheme does a reasonably good job of representing the discontinuity near the upper right (enlarged figure shown) with minor oscillations. The UW2-2D and UW4-2D schemes show some oscillations at this discontinuity but these are relatively mild. 

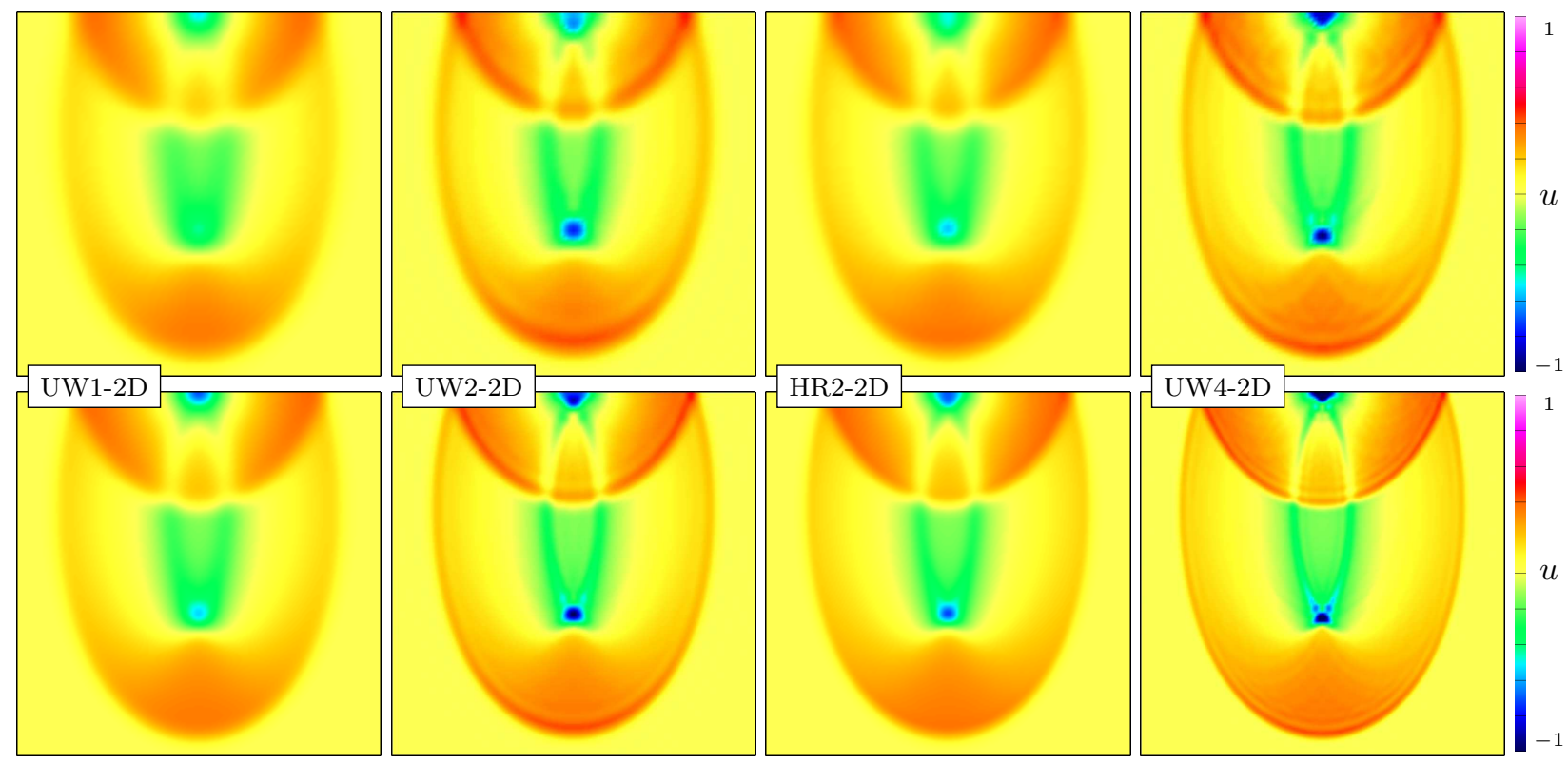

Figure 23: Two-dimensional top-hat: Contours of $u$ at $t=3$ for the four schemes; from left to right: first-order accurate UW1-2D, second-order accurate UW2-2D, high-resolution HR-2D, and fourth-order accurate UW4-2D. At top are results using 100 points in each coordinate direction and at bottom are results using 200.

\section{Conclusions}

We have presented a methodology to construct upwind discretizations for the wave equation in secondorder form that avoids reformulating the problem as a first-order hyperbolic system. To motivate the approach, we demonstrated how a first-order accurate upwind scheme could be developed by using the d'Alembert solution of the second-order wave equation to exactly advance a piecewise smooth representation of the solution. The piecewise smooth solution at the new time was then defined by integrating the exact solution over a cell.

To generalize the approach we showed how the solution to a local Riemann problem at cell faces can be used to define the upwind flux on the face given approximations to left and right biased states on the face. This localized form is the key ingredient needed for generalizing the scheme to multiple space dimensions, high-order accuracy, variable coefficients and systems of equations (we leave variable coefficients and systems to future work). Using the localized form of the upwind flux we then showed how to develop efficient spacetime schemes in one or more space dimensions to arbitrary order of accuracy. A method-of-lines approach could also be used, although we did not pursue that approach here.

In one space dimension we developed and analyzed schemes with orders of accuracy one, two, four and six. A second-order accurate nonlinear high-resolution scheme based on limiters was also developed. Normal mode analysis was used to determine the accuracy of the schemes and the stability bound on the time-steps. Modified equations were used to elucidate the dissipative and dispersive nature of the upwind schemes. The relationships between the modified equations, the solution error and the accuracy requirements in terms of points per wavelength were also described. In two dimensions we developed and analyzed schemes with orders of accuracy one, two and four along with a second-order accurate high-resolution scheme. A series of numerical tests confirmed the theoretical results and also demonstrated some of the attractive properties of the schemes.

There are a variety of avenues to consider in future work. Two important areas are the extension of the approach to variable coefficients and to systems of second-order wave equations such as Maxwell's equations written in second-order form [11], or the elastic wave equation and nonlinear extensions thereof. To address geometric complexity we will develop approximations for curvilinear grids which can be used in the context of overlapping grids [20] to develop efficient schemes for complex geometry. As shown in [10] for the case of the first-order system for linear elasticity, upwind schemes can be very effective when solving wave-propagation problems on overlapping grids since they naturally suppress possible instabilities that can be generated from the overlapping grid interpolation. Upwind schemes for second-order wave equations may also be useful 

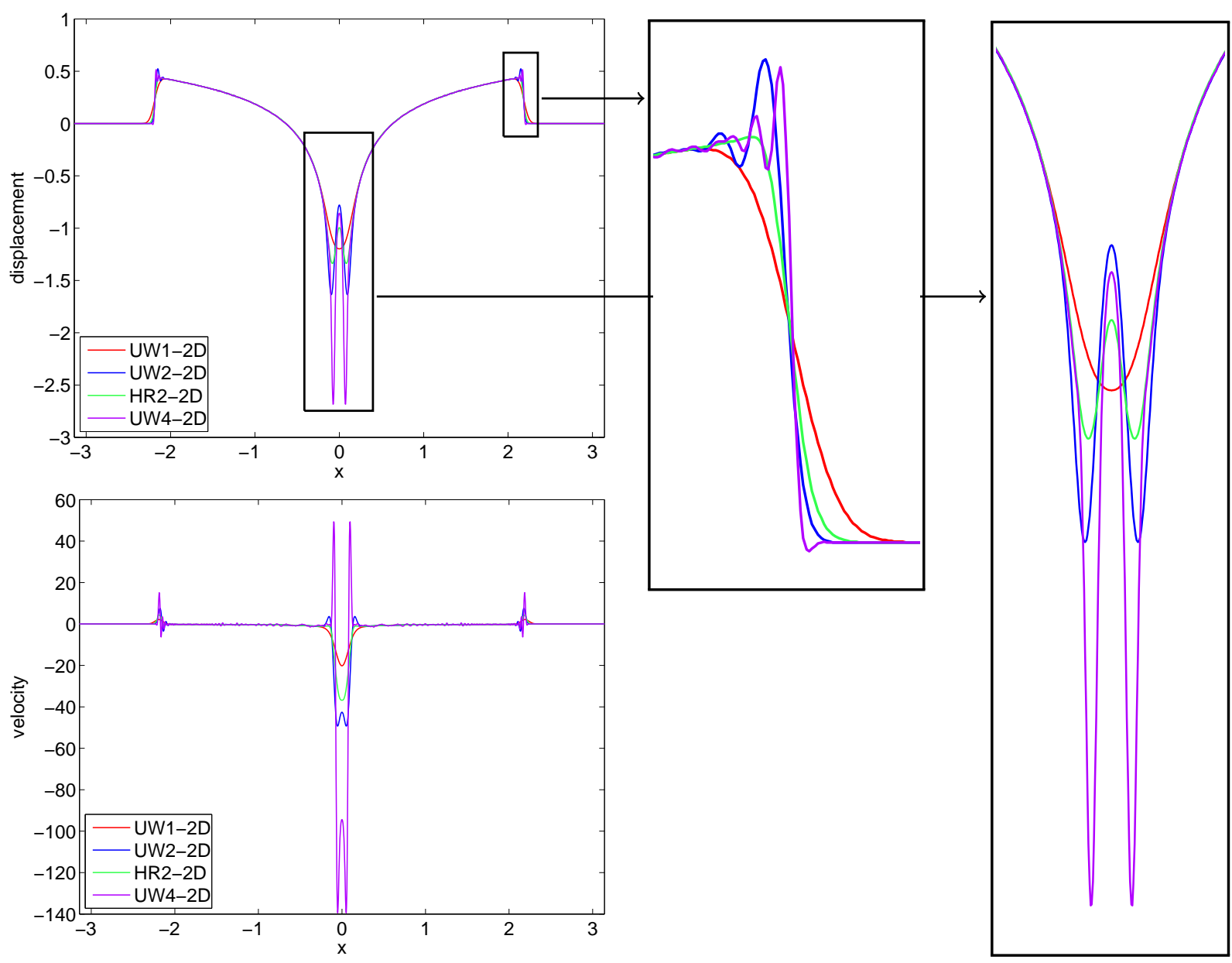

Figure 24: Two-dimensional top-hat: line plots of the displacement and velocity on the top surface $y=0$, at $t=3$ for the schemes UW1-2D, UW2-2D, HR2-2D and UW4-2D. All simulations were performed using 1600 points in each coordinate direction.

when combined with the embedded boundary approach to represent complex geometry [21, 22].

\section{Appendix A. High-order accurate stencils}

\section{Appendix A.1. Fourth-order accurate scheme}

The fourth-order accurate scheme in one-dimension can be written in the form of a stencil operation,

$$
\begin{aligned}
u_{i}^{n+1} & =\sum_{k=1}^{7}\left[C_{u, u}^{(4)}(k) u_{i-4+k}^{n}+C_{u, v}^{(4)}(k) v_{i-4+k}^{n}\right] \\
v_{i}^{n+1} & =\sum_{k=1}^{7}\left[C_{v, u}^{(4)}(k) u_{i-4+k}^{n}+C_{v, v}^{(4)}(k) v_{i-4+k}^{n}\right] .
\end{aligned}
$$

The stencil coefficients for the fourth-order scheme are defined as

$$
C_{u, u}^{(4)}=\left[\begin{array}{c}
\frac{1}{432}\left(-9+2 \lambda^{2}\right) \lambda^{3} \\
-\frac{1}{72}\left(3-9 \lambda-3 \lambda^{2}+2 \lambda^{3}\right) \lambda^{2} \\
\frac{1}{144}\left(96-45 \lambda-24 \lambda^{2}+10 \lambda^{3}\right) \lambda^{2} \\
\frac{1}{108}\left(108-135 \lambda^{2}+45 \lambda^{3}+27 \lambda^{4}-10 \lambda^{5}\right) \\
\frac{1}{144}\left(96-45 \lambda-24 \lambda^{2}+10 \lambda^{3}\right) \lambda^{2} \\
-\frac{1}{72}\left(3-9 \lambda-3 \lambda^{2}+2 \lambda^{3}\right) \lambda^{2} \\
\frac{1}{432}\left(-9+2 \lambda^{2}\right) \lambda^{3}
\end{array}\right] \quad C_{u, v}^{(4)}=\left[\begin{array}{c}
-\frac{1}{576 c} \Delta x \lambda^{2}\left(-5+4 \lambda^{2}\right) \\
\frac{1}{864 c} \Delta x \lambda^{2}\left(-45-12 \lambda+36 \lambda^{2}+8 \lambda^{3}\right) \\
-\frac{1}{1728 c} \Delta x \lambda^{2}\left(-225-384 \lambda+180 \lambda^{2}+64 \lambda^{3}\right) \\
\frac{1}{144 c} \lambda \Delta x\left(144-25 \lambda-60 \lambda^{2}+20 \lambda^{3}+8 \lambda^{4}\right) \\
-\frac{1}{1728 c} \Delta x \lambda^{2}\left(-225-384 \lambda+180 \lambda^{2}+64 \lambda^{3}\right) \\
\frac{1}{864 c} \Delta x \lambda^{2}\left(-45-12 \lambda+36 \lambda^{2}+8 \lambda^{3}\right) \\
-\frac{1}{576 c} \Delta x \lambda^{2}\left(-5+4 \lambda^{2}\right)
\end{array}\right]
$$


and

$$
C_{v, u}^{(4)}=\left[\begin{array}{c}
\frac{1}{48 \Delta x} c \lambda^{2}\left(-3+\lambda^{2}\right) \\
-\frac{1}{24 \Delta x} c \lambda\left(2-9 \lambda-4 \lambda^{2}+3 \lambda^{3}\right) \\
\frac{1}{48 \Delta x} c \lambda\left(64-45 \lambda-32 \lambda^{2}+15 \lambda^{3}\right) \\
-\frac{1}{12 \Delta x} c \lambda\left(30-15 \lambda-12 \lambda^{2}+5 \lambda^{3}\right) \\
\frac{1}{48 \Delta x} c \lambda\left(64-45 \lambda-32 \lambda^{2}+15 \lambda^{3}\right) \\
-\frac{1}{24 \Delta x} c \lambda\left(2-9 \lambda-4 \lambda^{2}+3 \lambda^{3}\right) \\
\frac{1}{48 \Delta x} c \lambda^{2}\left(-3+\lambda^{2}\right)
\end{array}\right] \quad C_{v, v}^{(4)}=\left[\begin{array}{c}
-\frac{1}{288} \lambda\left(-5+8 \lambda^{2}\right) \\
\frac{1}{48} \lambda\left(-5-2 \lambda+8 \lambda^{2}+2 \lambda^{3}\right) \\
-\frac{1}{96} \lambda\left(-25-64 \lambda+40 \lambda^{2}+16 \lambda^{3}\right) \\
\frac{1}{72}\left(72-25 \lambda-90 \lambda^{2}+40 \lambda^{3}+18 \lambda^{4}\right) \\
-\frac{1}{96}\left(-25-64 \lambda+40 \lambda^{2}+16 \lambda^{3}\right) \lambda \\
\frac{1}{48} \lambda\left(-5-2 \lambda+8 \lambda^{2}+2 \lambda^{3}\right) \\
-\frac{1}{288} \lambda\left(-5+8 \lambda^{2}\right)
\end{array}\right]
$$

where $\lambda=\frac{c \Delta t}{\Delta x}$.

\section{Appendix A.2. Sixth-order accurate scheme}

The sixth-order accurate scheme in one-dimension can be written in the form of a stencil operation,

$$
\begin{aligned}
u_{i}^{n+1} & =\sum_{k=1}^{9}\left[C_{u, u}^{(6)}(k) u_{i-5+k}^{n}+C_{u, v}^{(6)}(k) v_{i-5+k}^{n}\right] \\
v_{i}^{n+1} & =\sum_{k=1}^{9}\left[C_{v, u}^{(6)}(k) u_{i-5+k}^{n}+C_{v, v}^{(6)}(k) v_{i-5+k}^{n}\right] .
\end{aligned}
$$

The stencil coefficients for the sixth-order scheme are defined as

$$
\begin{gathered}
\frac{1}{288000}\left(1400-400 \lambda^{2}+29 \lambda^{4}\right) \lambda^{3} \\
-\frac{1}{36000}\left(-200+1400 \lambda+250 \lambda^{2}-400 \lambda^{3}-50 \lambda^{4}+29 \lambda^{5}\right) \lambda^{2} \\
\frac{1}{72000}\left(-5400+9800 \lambda+6000 \lambda^{2}-2800 \lambda^{3}-600 \lambda^{4}+203 \lambda^{5}\right) \lambda^{2} \\
-\frac{1}{36000}\left(-27000+9800 \lambda+9750 \lambda^{2}-2800 \lambda^{3}-750 \lambda^{4}+203 \lambda^{5}\right) \lambda^{2} \\
\frac{1}{28800}\left(28800-39200 \lambda^{2}+9800 \lambda^{3}+11200 \lambda^{4}-2800 \lambda^{5}-800 \lambda^{6}+203 \lambda^{7}\right) \\
-\frac{1}{36000}\left(-27000+9800 \lambda+9750 \lambda^{2}-2800 \lambda^{3}-750 \lambda^{4}+203 \lambda^{5}\right) \lambda^{2} \\
\frac{1}{72000}\left(-5400+9800 \lambda+6000 \lambda^{2}-2800 \lambda^{3}-600 \lambda^{4}+203 \lambda^{5}\right) \lambda^{2} \\
-\frac{1}{36000}\left(-200+1400 \lambda+250 \lambda^{2}-400 \lambda^{3}-50 \lambda^{4}+29 \lambda^{5}\right) \lambda^{2} \\
\frac{1}{288000}\left(1400-400 \lambda^{2}+29 \lambda^{4}\right) \lambda^{3} \\
-\frac{1}{34560 c} \Delta x\left(62-43 \lambda^{2}+10 \lambda^{4}\right) \lambda^{2} \\
C_{u, v}^{(6)}=\left[\begin{array}{c}
\left.1000 \lambda^{4}+87 \lambda^{5}\right) \lambda^{2} \\
\frac{1}{432000 c} \Delta x\left(6200+800 \lambda-4300 \lambda^{2}-600 \lambda^{3}+1000 \lambda^{2}\right. \\
-\frac{1}{216000 c} \Delta x\left(10850+5400 \lambda-7525 \lambda^{2}-3600 \lambda^{3}+1750 \lambda^{4}+261 \lambda^{5}\right) \lambda^{2} \\
\frac{1}{86400 c} \Delta x\left(8680+21600 \lambda-6020 \lambda^{2}-4680 \lambda^{3}+1400 \lambda^{4}+261 \lambda^{5}\right) \lambda^{2} \\
\frac{1}{86400 c} \Delta x\left(-86400+10850 \lambda+39200 \lambda^{2}-7525 \lambda^{3}-6720 \lambda^{4}+1750 \lambda^{5}+348 \lambda^{6}\right) \lambda \\
\frac{1}{86400 c} \Delta x\left(8680+21600 \lambda-6020 \lambda^{2}-4680 \lambda^{3}+1400 \lambda^{4}+261 \lambda^{5}\right) \lambda^{2} \\
-\frac{1}{216000 c} \Delta x\left(10850+5400 \lambda-7525 \lambda^{2}-3600 \lambda^{3}+1750 \lambda^{4}+261 \lambda^{5}\right) \lambda^{2} \\
\frac{1}{432000 c} \Delta x\left(6200+800 \lambda-4300 \lambda^{2}-600 \lambda^{3}+1000 \lambda^{4}+87 \lambda^{5}\right) \lambda^{2} \\
-\frac{1}{34560 c} \Delta x\left(62-43 \lambda^{2}+10 \lambda^{4}\right) \lambda^{2}
\end{array}\right]
\end{gathered}
$$

and

$$
C_{v, u}^{(6)}=\left[\begin{array}{c}
\frac{1}{1440 \Delta x} c\left(21-10 \lambda^{2}+\lambda^{4}\right) \lambda^{2} \\
-\frac{1}{360 \Delta x} c\left(-4+42 \lambda+10 \lambda^{2}-20 \lambda^{3}-3 \lambda^{4}+2 \lambda^{5}\right) \lambda \\
\frac{1}{360 \Delta x} c\left(-54+147 \lambda+120 \lambda^{2}-70 \lambda^{3}-18 \lambda^{4}+7 \lambda^{5}\right) \lambda \\
-\frac{1}{360 \Delta x} c\left(-540+294 \lambda+390 \lambda^{2}-140 \lambda^{3}-45 \lambda^{4}+14 \lambda^{5}\right) \lambda \\
\frac{1}{144 \Delta x} c\left(-392+147 \lambda+224 \lambda^{2}-70 \lambda^{3}-24 \lambda^{4}+7 \lambda^{5}\right) \lambda \\
-\frac{1}{360 \Delta x} c\left(-540+294 \lambda+390 \lambda^{2}-140 \lambda^{3}-45 \lambda^{4}+14 \lambda^{5}\right) \lambda \\
\frac{1}{360 \Delta x} c\left(-54+147 \lambda+120 \lambda^{2}-70 \lambda^{3}-18 \lambda^{4}+7 \lambda^{5}\right) \lambda \\
-\frac{1}{360 \Delta x} c\left(-4+42 \lambda+10 \lambda^{2}-20 \lambda^{3}-3 \lambda^{4}+2 \lambda^{5}\right) \lambda \\
\frac{1}{1440 \Delta x} c\left(21-10 \lambda^{2}+\lambda^{4}\right) \lambda^{2}
\end{array}\right]
$$




$$
C_{v, v}^{(6)}=\left[\begin{array}{c}
-\frac{1}{8640}\left(31-43 \lambda^{2}+15 \lambda^{4}\right) \lambda \\
\frac{1}{2160}\left(62+12 \lambda-86 \lambda^{2}-15 \lambda^{3}+30 \lambda^{4}+3 \lambda^{5}\right) \lambda \\
-\frac{1}{2160}\left(217+162 \lambda-301 \lambda^{2}-180 \lambda^{3}+105 \lambda^{4}+18 \lambda^{5}\right) \lambda \\
\frac{1}{2160}\left(434+1620 \lambda-602 \lambda^{2}-585 \lambda^{3}+210 \lambda^{4}+45 \lambda^{5}\right) \lambda \\
\frac{1}{864}\left(864-217 \lambda-1176 \lambda^{2}+301 \lambda^{3}+336 \lambda^{4}-105 \lambda^{5}-24 \lambda^{6}\right) \\
\frac{1}{2160}\left(434+1620 \lambda-602 \lambda^{2}-585 \lambda^{3}+210 \lambda^{4}+45 \lambda^{5}\right) \lambda \\
-\frac{1}{2160}\left(217+162 \lambda-301 \lambda^{2}-180 \lambda^{3}+105 \lambda^{4}+18 \lambda^{5}\right) \lambda \\
\frac{1}{2160}\left(62+12 \lambda-86 \lambda^{2}-15 \lambda^{3}+30 \lambda^{4}+3 \lambda^{5}\right) \lambda \\
-\frac{1}{8640}\left(31-43 \lambda^{2}+15 \lambda^{4}\right) \lambda
\end{array}\right]
$$

where $\lambda=\frac{c \Delta t}{\Delta x}$.

\section{References}

[1] G. Efraimsson, G. Kreiss, A note on the effect of artificial viscosity on solutions of conservation laws, Applied Numerical Mathematics 21 (2) (1996) 155 - 173.

URL http://www.sciencedirect.com/science/article/pii/0168927496000062

[2] R. C. Swanson, R. Radespiel, E. Turkel, On some numerical dissipation schemes, Journal of Computational Physics 147 (1998) 518-544.

[3] S. K. Godunov, Finite difference method for numerical computation of discontinuous solutions of the equations of fluid dynamics, Mat. Sb. 47 (1959) 271-306.

[4] J. P. Boris, D. L. Book, Flux-corrected transport. I. SHASTA, a fluid transport algorithm that works, J. Comput. Phys. 11 (1973) 38-69.

[5] P. Colella, P. R. Woodward, The piecewise parabolic method (PPM) for gas-dynamical simulations, J. Comput. Phys. 54 (1) (1984) 174-201.

[6] A. Harten, B. Engquist, S. Osher, S. Chakravarthy, Uniformly high order accurate essentially nonoscillitory schemes, III, J. Comput. Phys. 71 (1987) 231-303.

[7] C.-W. Shu, S. Osher, Efficient implementation of essentially non-oscillatory shock-capturing schemes, J. Comput. Phys. 77 (2) (1988) 439-471.

[8] B. Cockburn, C. Shu, TVB Runge-Kutta local projection discontinuous Galerkin finite-element method for conservation-laws 2: general framework, Math. Comput. 52 (1989) 411-435.

[9] G.-S. Jiang, C.-W. Shu, Efficient implementation of weighted ENO schemes, J. Comput. Phys. 126 (1) (1996) 202-228.

[10] D. Appelö, J. W. Banks, W. D. Henshaw, D. W. Schwendeman, Numerical methods for solid mechanics on overlapping grids: Linear elasticity (LLNL-JRNL-422223), submitted for publication.

[11] W. D. Henshaw, A high-order accurate parallel solver for Maxwell's equations on overlapping grids, SIAM J. Sci. Comput. 28 (5) (2006) 1730-1765.

URL http://link.aip.org/link/?SCE/28/1730/1

[12] G. B. Whitham, Linear and Nonlinear Waves, Wiley-Interscience, New York, 1974.

[13] R. J. Leveque, Finite Volume Methods for Hyperbolic Problems, Cambridge University Press, Cambridge, 2004.

[14] P. Lax, B. Wendroff, Systems of conservation laws, Comm. Pure and Appl. Math 13 (1960) 217-237.

[15] B. Gustafsson, H.-O. Kreiss, A. Sundström, Stability theory of difference approximations for mixed initial boundary value problems. II, Math. Comput. 26 (119) (1972) 649-686. 
[16] A. Harten, High resolution schemes for hyperbolic conservation laws, Journal of Computational Physics 49 (3) (1983) $357-393$.

URL http://www.sciencedirect.com/science/article/pii/0021999183901365

[17] H.-O. Kreiss, J. Oliger, Comparison of accurate methods for the integration of hyperbolic equations, Tellus 24 (1972) 199-215.

[18] B. Gustafsson, H.-O. Kreiss, J. Oliger, Time Dependent Problems and Difference Methods, John Wiley and Sons Inc., 1995.

[19] J. W. Banks, T. Aslam, W. J. Rider, On sub-linear convergence for linearly degenerate waves in capturing schemes, J. Comput. Phys. 227 (14) (2008) 6985-7002.

[20] G. S. Chesshire, W. D. Henshaw, Composite overlapping meshes for the solution of partial differential equations, J. Comput. Phys. 90 (1) (1990) 1-64.

[21] H.-O. Kreiss, N. A. Petersson, J. Yström, Difference approximations for the second order wave equation, SIAM J. Numer. Anal. 40 (5) (2002) 1940-1967.

[22] H.-O. Kreiss, N. A. Petersson, J. Yström, Difference approximations of the Neumann problem for the second order wave equation, SIAM J. Numer. Anal. 42 (3) (2004) 1292-1323.

Prepared by LLNL under Contract DE-AC52-07NA27344. 